\title{
Parametric Testing of a DWPF Glass
}

\author{
F. Bazan \\ J. Rego
}

Manuscript date: March 1985

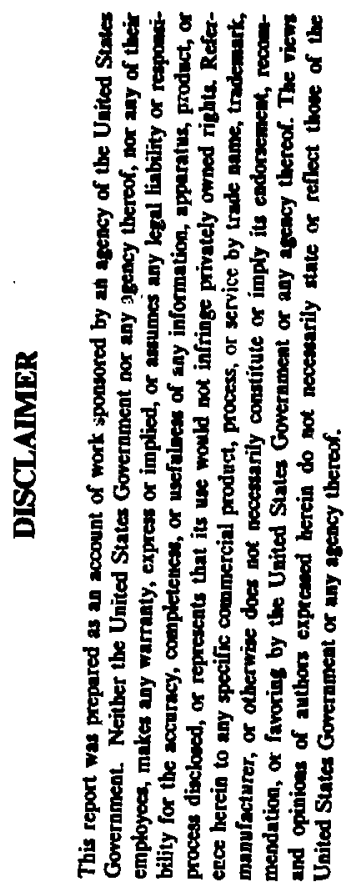

\section{LAWRENCE LIVERMORE NATIONAL LABORATORY} University of California - Livermore, California - 94550

Available from: National Technical Information Service • U.S. Department of Commetce 5285 Port Royal Road • Springfield, VA 22161 -\$11.50 per copy • (Microfiche \$4.50)

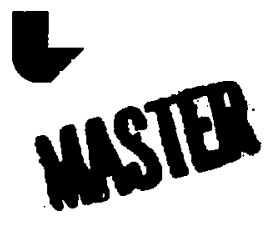




\section{Contents}

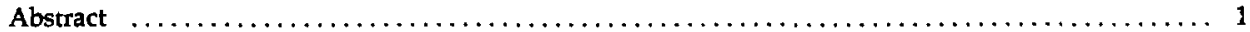

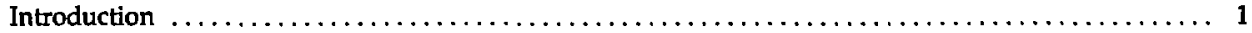

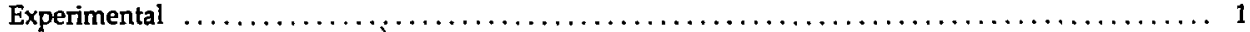

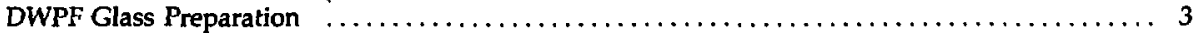

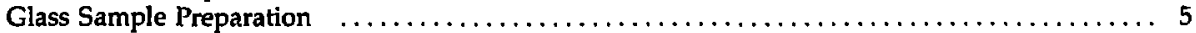

Rock Sample Preparation $\ldots \ldots \ldots \ldots \ldots \ldots \ldots \ldots \ldots \ldots \ldots \ldots \ldots \ldots \ldots \ldots \ldots \ldots \ldots \ldots$

Stainless Steel Supports $\ldots \ldots \ldots \ldots \ldots \ldots \ldots \ldots \ldots \ldots \ldots \ldots \ldots \ldots \ldots \ldots \ldots \ldots \ldots \ldots$

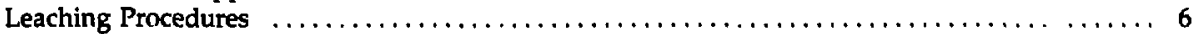

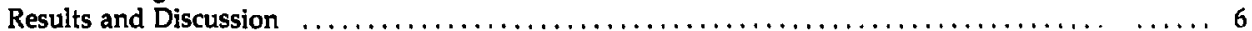

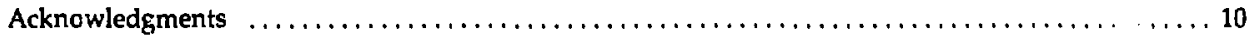

References $\ldots \ldots \ldots \ldots \ldots \ldots \ldots \ldots \ldots \ldots \ldots \ldots \ldots \ldots \ldots \ldots \ldots \ldots \ldots \ldots \ldots \ldots \ldots \ldots \ldots \ldots 10$

Appendix A. Test I: DWPF Glass - Deionized Water Experiment,

Monolith Examples $\ldots \ldots \ldots \ldots \ldots \ldots \ldots \ldots \ldots \ldots \ldots \ldots \ldots \ldots \ldots \ldots \ldots \ldots \ldots \ldots \ldots \ldots \ldots$

Appendix B. Test I: DWPF Glass - Deionized Water Experiment,

Crushed Samples $\ldots \ldots \ldots \ldots \ldots \ldots \ldots \ldots \ldots \ldots \ldots \ldots \ldots \ldots \ldots \ldots \ldots \ldots \ldots \ldots \ldots \ldots 29$

Appendix C. Test II: DWPF Glass - J-13 Water Experiment,

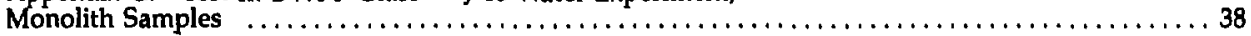

Appendix D. Test III: DWPF Glass - J-13 Water + Tuff Experiment,

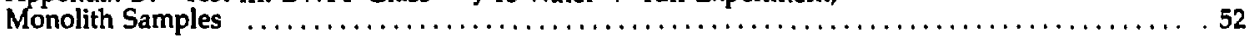

Appendix E. Test IV: DWPF Glass - J-13 Water + Tuff + Stainless Steel

Experiment, Monolith Samples 


\title{
Parametric Testing of a DWPF Glass
}

\begin{abstract}
A series of tests has been performed to characterize the chemical stability of a DWPF borosilicate glass sample as part of the Waste Package Task of the Nevada Nuclear Waste Storage Investigations (NNWSI) Project. This material was prepared at the Savannah River Laboratory for the purpose of testing the 165-frit matrix doped with a simulated nonradioactive waste. All tests were conducted at $90^{\circ} \mathrm{C}$ using deionized water and J-13 water (a tuffaceous formation ground water). In the deionized water tests, both monoliths and crushed glass were tested at various ratios of surface area of the sample to volume of water in order to compare leach rates for different sample geometries or leaching times. Effects on the leach rates as a reult of the presence of crushed tuff and stainless steel material were also investigated in the tests with J-13 water.
\end{abstract}

\section{Introduction}

Parametric testing is important because it helps us to understand the behavior of a particular waste form under different experimental conditions. By optimizing these conditions, one can presumably design relatively shori tests to establish the chemical stability of waste forms such as the DWPF borosilicate glass. The parameters investigated were leachant composition, ratios of waste-form surface area to water volume (SA/V), effects of the presence of crushed tuff in some tests and of crushed tuff and stainless steel in others, and leaching times ranging from 1 to 56 days. All tests were conducted at $90^{\circ} \mathrm{C}$ and were of a static nature.

Four series of leaching tests were conducted.
These were:

1. Glass monoliths and crushed glass with deionized water at $S A / V$ ratios of $0.1,0.3$, 0.5 , and $1.0 \mathrm{~cm}^{-1}$ for $1,3,7,14,28$, and 56 days.

11. Glass monoliths with $\mathrm{J}-13$ water at SA/V ratios of 0.3 , and $0.5 \mathrm{~cm}^{-1}$ for $3,7,14,28$, and 56 days.

1Il. Glass monoliths with J-13 water and crushed tuff at SA/V ratios of 0.1 and 0.3 $\mathrm{cm}^{-1}$ for $7,14,28,56,91$, and 182 days.

IV. Glass monoliths with J-13 water, crushed tuff, and stainless steel at SA/V ratios of 0.3 and $0.5 \mathrm{~cm}-1$ for $3,7,14,28,56$, and 91 days.

\section{Experimental}

To perform the parametric testing, the following materials were obtained: a borosilicate glass (DWPF) from the Savannah River Laboratory, crushed Topopah Spring tuff rock from an outcrop located at Fran Ridge (Nevada Test Site), J-13 ground water from a well near the Yucca Mountain Site at the Nevada Test Site, and 304 L stainless steel coupons. The compositions of the glass, tuff, and J-13 ground water are given in Tables 1 , 2 , and 3, respectively. The rock and water localities and composition are described by Knauss (1984) and Oversby (1984).
Table 1 lists the nominal composition of the glass as supplied to LLNL by SRL and the average of the results of electron microprobe analyses performed at LLNL on five samples of the glass. For each sample, 10 spot analyses were performed and the data were averaged. The uncertainty estimates in Table 1 are based on the standard deviation of the average of the five sample averages. The SRL data giving the results of a partial analysis by $x$-ray fluorescence are also shown for comparison. 
Table 1. DWPF glass (simulated waste) composition.

\begin{tabular}{|c|c|c|c|}
\hline Component & $\underset{\text { (wt\%) }}{\text { Nominal }}$ & $\begin{array}{c}\text { LLNL }^{b} \\
(w t \%)\end{array}$ & $\begin{array}{c}\mathrm{SRL}^{2, c} \\
(w t \%)\end{array}$ \\
\hline $\mathrm{SiO}_{2}$ & 53.47 & $54.9 \pm 2.1$ & \\
\hline $\mathrm{Na}_{2} \mathrm{O}$ & 11.40 & $9.74 \pm 0.54$ & \\
\hline $\mathrm{Li}_{2} \mathrm{O}$ & 5.04 & & \\
\hline $\mathrm{B}_{2} \mathrm{O}_{3}$ & 7.20 & & \\
\hline $\mathrm{MgO}$ & 0.72 & $0.72 \pm 0.04$ & 0.65 \\
\hline $\mathrm{ZrO}_{2}$ & 0.72 & $0.66 \pm 0.07$ & 0.58 \\
\hline $\mathrm{Fe}_{2} \mathrm{O}_{3}$ & 10.53 & $10.6 \pm 0.7$ & 10.2 \\
\hline $\mathrm{MnO}_{2}$ & 2.35 & $2.72 \pm 0.13$ & 2.6 \\
\hline $\mathrm{CaO}$ & 1.37 & $1.51 \pm 0.32$ & 1.2 \\
\hline Nio & 1.06 & $1.84 \pm 0.24$ & 0.83 \\
\hline $\mathrm{Al}_{2} \mathrm{O}_{3}$ & 3.78 & $5.13 \pm 0.18$ & \\
\hline Zeolite & 1.68 & & \\
\hline $\mathrm{CeO}_{2}$ & 0.42 & & \\
\hline $\mathrm{K}_{2} \mathrm{O}$ & 0.14 & & \\
\hline SrO & 0.10 & & \\
\hline $\mathrm{RuO}_{2}$ & 0.036 & & \\
\hline $\mathrm{Cs}_{2} \mathrm{O}$ & 0.0028 & & \\
\hline
\end{tabular}

Data from Bibler, SRL, pernonal communication.

b Lawrence Livermore National Laboratory.

' Savannah River Laboratory.
Table 2. Topopah Spring Tuff composition. ${ }^{2}$

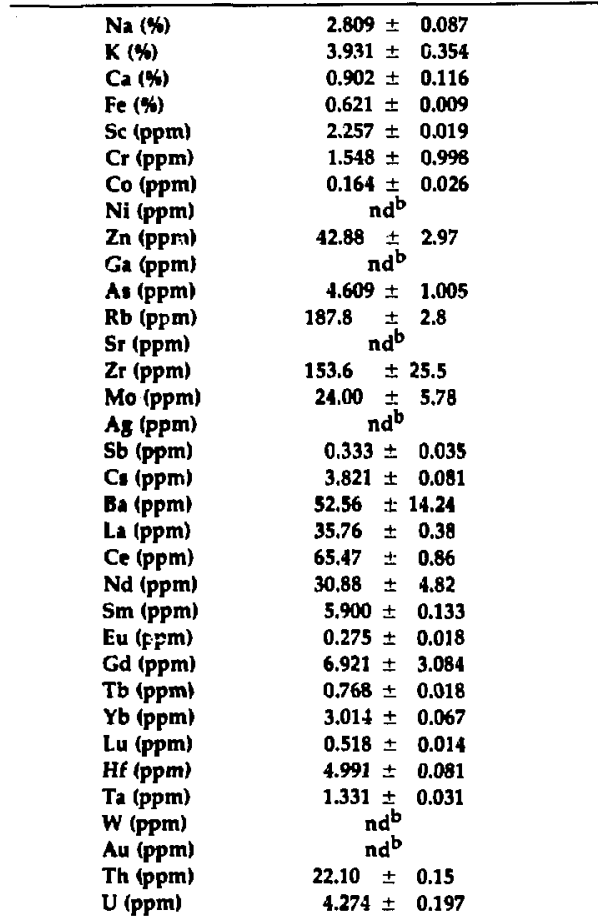

\footnotetext{
Neutron Activation Data from K. G. Knauss, UCRL 53558 (1994).

b nd = no data.
}

Table 3. J-13 Ground water chemical composition.

\begin{tabular}{|c|c|c|c|c|c|c|}
\hline Element & \multicolumn{3}{|c|}{ Non-equilibrated (mg/l) } & \multicolumn{3}{|c|}{ Equilibraled (mg/l) } \\
\hline & & & & & & \\
\hline AI & 0.372 & $<0.008$ & $<0.008$ & 0.512 & 0.011 & 0.012 \\
\hline $\mathbf{B}$ & $0.14 B$ & 0.127 & 0.131 & 0.220 & 0.228 & 0.227 \\
\hline $\mathrm{Cu}$ & 0.036 & 0.069 & 0.021 & 0.011 & 0.035 & 0.038 \\
\hline Fe & 0.017 & 0.007 & 0.004 & 0.008 & 0.059 & 0.013 \\
\hline $\mathbf{L i}$ & 0.035 & 0.039 & 0.041 & 0.046 & 0.046 & 0.046 \\
\hline Mn & $<0.001$ & $<0.001$ & $<0.001$ & 0.002 & 0.002 & 0.002 \\
\hline Mo & 0.024 & 0.013 & 0.012 & 0.012 & 0.011 & 0.012 \\
\hline $\mathbf{N i}$ & $<0.008$ & $<0.00$ & $<0.008$ & $<0.008$ & $<0.008$ & $<0.008$ \\
\hline $\mathbf{P}$ & 0.824 & $<0.056$ & $<0.056$ & 0.070 & 0.088 & 0.062 \\
\hline Pb & $<0.016$ & $<0.016$ & $<0.016$ & $<0.016$ & $<0.016$ & $<0.016$ \\
\hline $\mathbf{S i}$ & 27.8 & 25.6 & 26.3 & 38.9 & 38.8 & 38.3 \\
\hline $\mathbf{S r}$ & $<0.01$ & 0.022 & 0.021 & 0.047 & 0.035 & 0.038 \\
\hline $\mathbf{U}$ & $<0.06$ & $<0.06$ & $<0.06$ & $<0.06$ & $<0.06$ & $<0.06$ \\
\hline $\mathbf{Z n}$ & 0.034 & 0.009 & $<0.005$ & 0.026 & 0.029 & 0.025 \\
\hline Ca & 11.7 & 12.2 & 12.9 & 12.0 & 12.3 & 12.8 \\
\hline $\mathbf{K}$ & 6.56 & 4.52 & 4.66 & 12.8 & 13.1 & 12.6 \\
\hline $\mathbf{M}$ & 1.80 & 1.85 & 1.89 & 0.153 & 0.153 & 0.155 \\
\hline $\mathbf{N a}$ & 42.1 & 40.9 & 43.6 & 46.6 & 47.6 & 46.7 \\
\hline
\end{tabular}




\section{DWPF Glass Preparation}

The glass sample was prepared by the Savannah River Laboratory under the direction of the Waste Solidification Technology Division (Bibler, 1983). The process was to slurry-feed a mixture of 165 -frit and simulated waste to a small jouleheated melter. The finul composition uras $72 \mathrm{wt} \%$ frit and $28 w t \%$ simulated waste. The glass was melted at $1150^{\circ} \mathrm{C}$ and poured into a $500-\mathrm{cm}^{3}$ stainless steel beaker with a surface temperature of approximately $600^{\circ} \mathrm{C}$. The filling process took approximately 4 hours, which corresponds to a glass residence time in the melter of 10 hours. No annealing procedures were performed. A photograph of the glass and stainless steel can is shown in Fig. 1. Removal of the glass sample from the stainless steel can was only possible after cutting

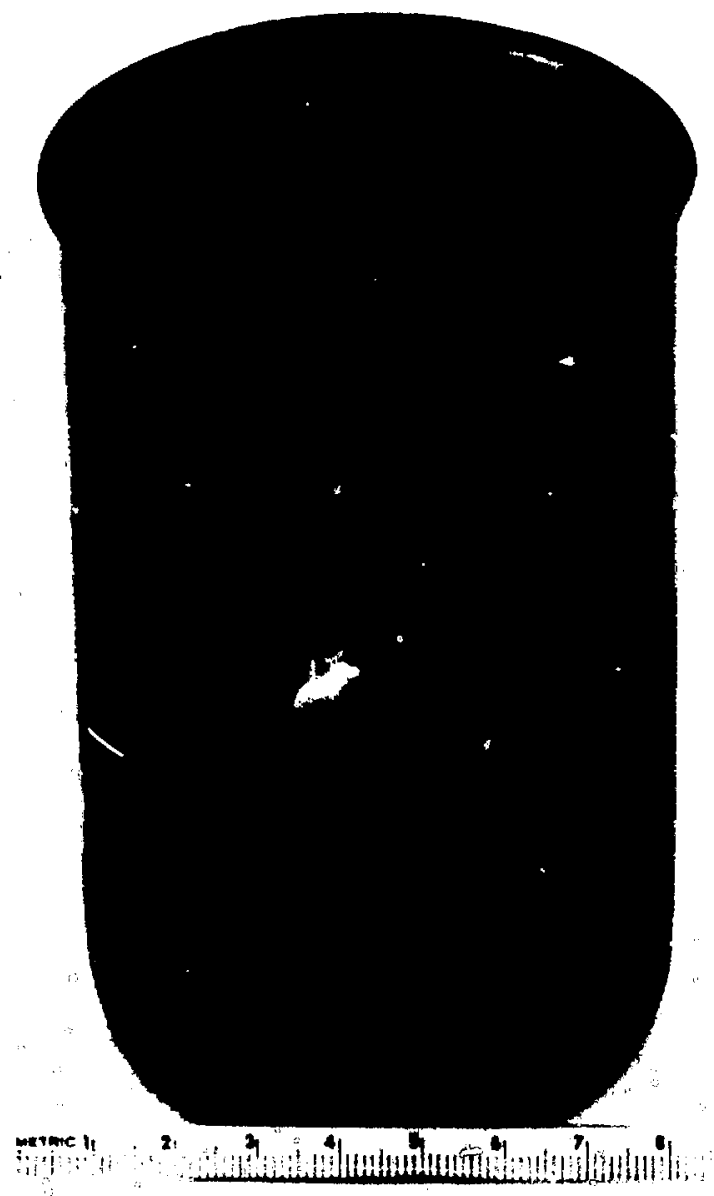

Figure 1. DWPF glass in steel can. 
the can along three different lengths, as shown in Fig. 2. During this procedure, the glass broke into several pieces, as shown in Fig. 3. These pieces were later used to prepare the monoliths for the various tests.

The glass was black to olive-gray in color with well-developed concoidal patterns. We also noticed locally developed banding alternating from black to olive-gray, and regions of frothy and vesicular mass at the top and center of the core. All surfaces were smooth, and there was no evidence of crystalline phenocrysts. Representative samples from the above were analyzed by electron microprobe; the results of these analyses are shown in Table 1 and indicate that, despite its physical appearance suggesting a heterogeneous chemical composition, the glass material was very uniform based on the electron microprobe results.

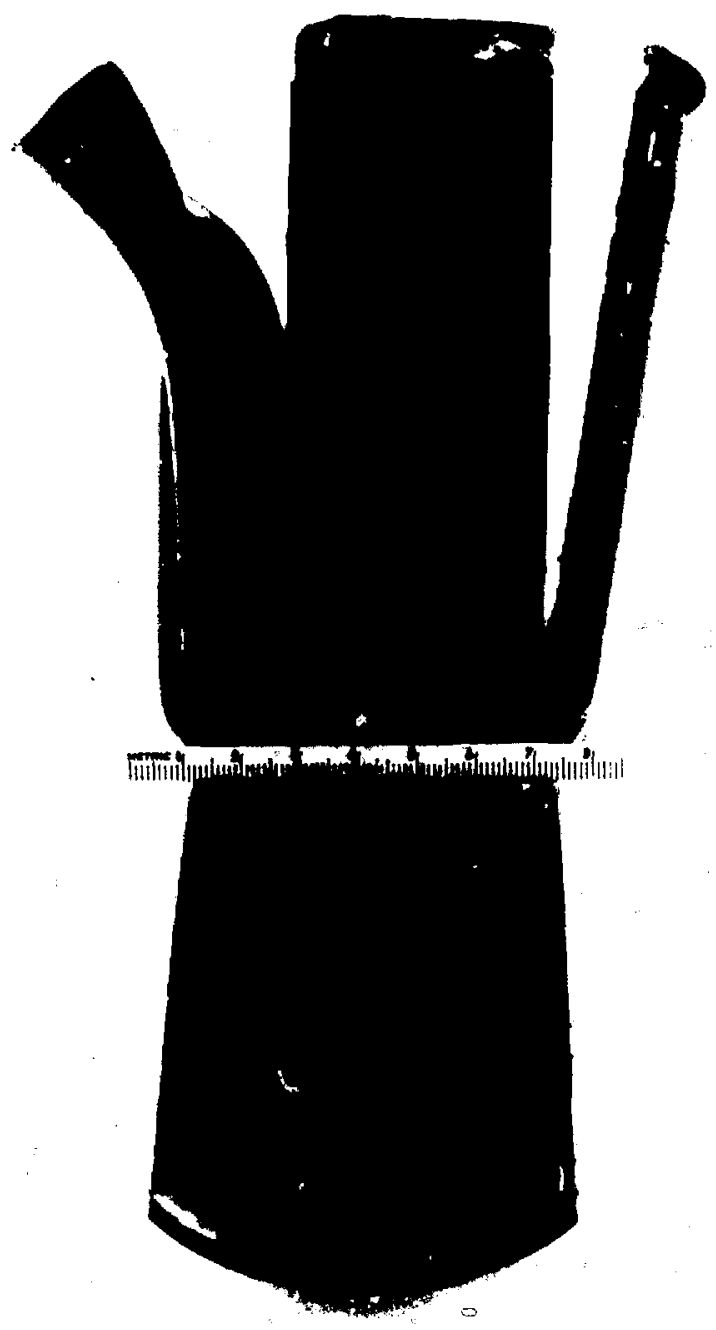

Figure 2. Stainless steel can. 


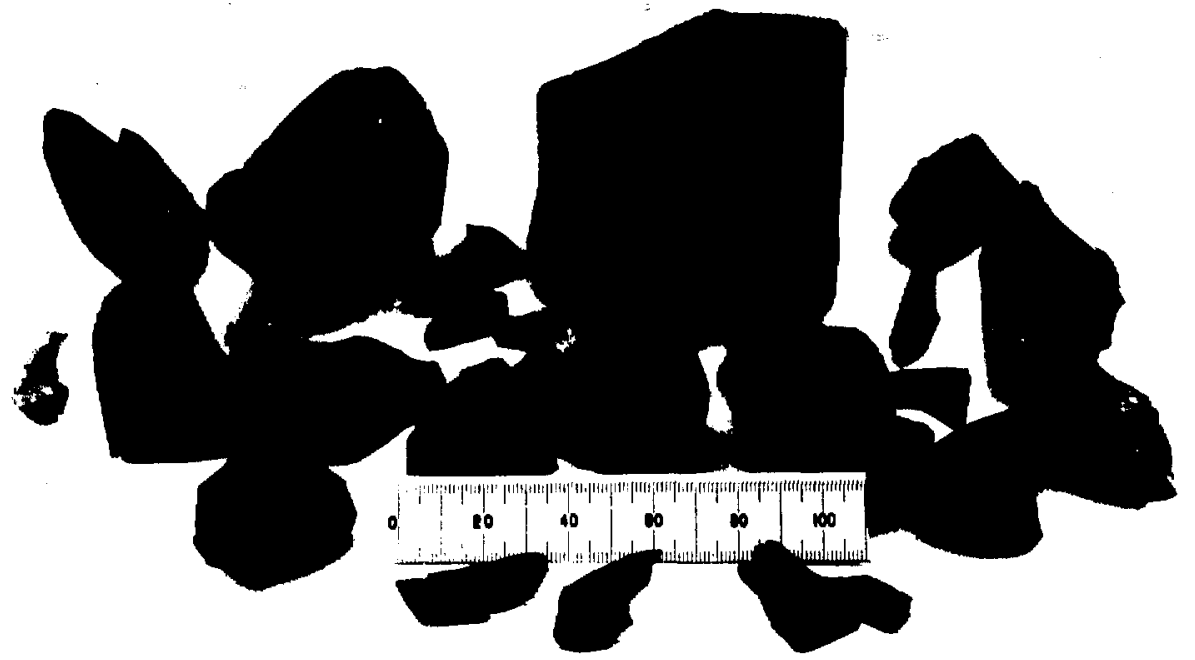

Figure 3. DWPF glass.

\section{Glass Sample Preparation}

Glass samples were prepared as monoliths and particles according to MCC-1 and MCC-3 procedures (Nuclear Waste Materials Handbook Test Methods, 1983). A saw with diamond wheel was used to cut monoliths in the form of rectangular prisms approximately $1 \times 2 \mathrm{~cm}$ long and 2$3 \mathrm{~mm}$ thick. Care was exercised not to use glass pieces that had been in contact with the stainless steel can. After cutting, the surface area of each monolith was measured with calipers, and then the glass was cleaned in an ultrasonic bath with deionized water and ethanol. Depending on the ratio of the specimen surface area to the leachant volume, samples with a surface area of 200-600 $\mathrm{mm}^{2}$ were used for the leaching.

The glass particles, $40-80$ mesh size, were obtained by crushing pieces of glass, not suitable for monolith cutting, in a steel mortar and pestle. The crushed particles were sieved with the appropriate size screens and also cleaned in an ultrasonic bath with water and ethanol. The surface area of the particles $n$ as calculated from the average spherical size of each particle $\left(298.5 \times 10^{-6} \mathrm{~m}\right)$ using the following algorithm:
$\mathrm{SA}=\frac{6 \mathrm{~m}_{\mathrm{s}}}{\rho \overline{\mathrm{D}}_{\mathrm{p}}}$

where

$\mathrm{m}_{\mathrm{s}}=$ mass of sample, $\mathrm{g}$,

$\rho=$ sample density $\mathrm{g} / \mathrm{m}^{3}$, and

$\overline{\mathrm{D}}_{\mathrm{p}}=$ mean particle diameter, $\mathrm{m}$.

\section{Rock Sample Preparation}

The crushed tuff material was prepared by crushing small pieces of outcrop rock with a small ceramic plate jaw crusher, followed by further crushing with a plate grinder. The plates are made of high purity alumina. The rock powder from the plate grinder was sieved through 60 and 100 mesh screens on a mechanical sieve shaker. Finally, the rock powder was homogenized in a drum mixer for 24 hours before use in the leaching tests (Oversby, 1984), and pretreated to remove the caliche. This procedure consisted of washing the crushed rock with deionized water, twice at room temperature by shaking, letting the tuff settle, and decanting the wash water, and once at $90^{\circ} \mathrm{C}$ by heating for 12 hours, letting it cool, and again decanting the wash water. 


\section{Stainless Steel Supports}

In the fourth series of parametric testing, stainless steel supports were used to hold the glass monoliths. These supports were made from $2.2 \times 2.2 \mathrm{~cm}$ squares of $0.16-\mathrm{cm}$-thick $304 \mathrm{~L}$ stainless steel coupons. The corners of the squares were bent downward to allow approximately $0.5 \mathrm{~cm}$ of space between the waste form and the crushed tuff resting at the bottom of the leaching container. Furthermore, the surface of the support was perforated with nine $0.318-\mathrm{cm}$-diameter holes to allow a free path for water mixing around the waste form.

\section{Leaching Procedures}

Following is the analytical procedure used to conduct the four series of parametric testing described in the introduction.

In Test I, monoliths were placed on Teflon supports during the leaching period following procedures recommended by the $\mathrm{MCC}-1$ leaching test (1983). In the case of the crushed glass experiments, the procedures were those recommended by the MCC-3 leaching test. In Test II, only monoliths were used in the same manner as in Test I. In Test III, the crushed tuff was placed on the base of the leaching container and the glass monoliths rested on Teflon supports above the tuff (there was no contact between glass and tuff). The ratio of J-13 water volume to tuff weight was $20 \mathrm{ml}$ to $1 \mathrm{~g}$. In Test IV, stainless steel supports were substituted for the Teflon supports, but the glass-tuff configuration was the same as in Test III. Appropriate blank samples (glass omitted) were run in each case to make background corrections.

The Teflon containers used in Tests I, II, and IlI were those recommended in the MCC-1 test $\left(40-\mathrm{cm}^{3}\right.$ capacity). The Teflon containers used in Test IV were $25 \mathrm{~cm}^{3}$ in capacity, but were made of the same material as the $40-\mathrm{cm}^{3}$ capsules used in the first three tests. The reduction in size was necessary because of the size of stainless steel support.

At the conclusion of each leaching period, the solutions were cooled and the $\mathrm{pH}$ was measured within a few hours. In the tests involving glass particles and crushed tuff, the soiutions were passed through a $45-\mu \mathrm{m}$ filter before measuring the $\mathrm{pH}$. The solutions were then acidified by adding concentrated ultrex nitric acid in amounts equal to $1 \%$ of the original volume. After acidification, all solutions were heated for 12 hours at $90^{\circ} \mathrm{C}$. At the end of this period, the solutions were cooled and aliquots were removed for ICP elemental analysis. Inductively complex plasma (ICP) data were obtained for lithium, boron, sodium, magnesium, aluminum, silicon, potassium, calcium, manganese, iron, nickel, strontium, and zirconium. These data are shown in Appendices A-E.

\section{Results and Discussion}

As we have described, four series of parametric tests were conducted to evaluate the chemical stability of the DWPF glass under different experimental conditions of leachant composition, ratios of surface area of the glass sample to leachant volume, and effects on leach rates caused by the addition of tuff and or stainless steel to the system. These four tests generated an enormous amount of data, as shown in Appendices A-E. However, only some of the results will be discussed in this report.

The results to be discussed in Test I are those shown for lithium, boron, sodium, and silicon in Appendices A and B. Normalized elemental leach rates were calculated using the elemental concentrations in the leachates as determined by ICP. The algorithm to calculate leach rates can be expressed as

$$
\begin{aligned}
& \text { leach rate }=\frac{\left(C_{x}\right)(V)}{\left(f_{x}\right)(S A)(t)} \\
& \text { where } \\
& \qquad \begin{aligned}
C_{x}= & \text { concentration of element } x \text { in leach solu- } \\
& \text { tion, } g / \mathrm{cm}^{3}, \\
V & =\text { volume of leach solution, } \mathrm{cm}^{3}, \\
f_{x} & =\text { mass fraction of element } x \text { in the un- } \\
& \text { leached specimen, } \\
S A & =\text { specimen surface area, } \mathrm{m}^{2}, \text { and } \\
t & =\text { leaching time, } d .
\end{aligned}
\end{aligned}
$$

The leach rate values are shown in Table 4 for the monolith samples and in Table 5 for the crushed glass samples. We have taken the leach rate values from Tables 4 and 5 for samples run at SA/V ratios of 0.3 and $0.5 \mathrm{~cm}^{-1}$ and compared them 
Table 4. Test I: DWPF glas (monoliths)deionized water.

\begin{tabular}{cccccc}
\hline & \multicolumn{5}{c}{ Normalized elemental leach rate $\left(\mathrm{g} / \mathbf{m}^{2} \cdot \mathbf{d}\right)$} \\
\cline { 2 - 6 } Leach time & SA/V & Li & B & Na & Si \\
\hline 1 & 0.1 & 0.95 & 0.66 & 0.90 & 0.93 \\
3 & 0.1 & 1.59 & 1.48 & 1.65 & 1.47 \\
7 & 0.1 & 1.07 & 1.01 & 1.12 & 0.93 \\
14 & 0.1 & 0.79 & 0.74 & 0.83 & 0.65 \\
28 & 0.1 & 0.50 & 0.48 & 0.53 & 0.40 \\
56 & 0.1 & 0.30 & 0.28 & 0.31 & 0.24 \\
1 & 0.3 & 1.61 & 1.45 & 1.66 & 1.44 \\
3 & 0.3 & 1.22 & 1.05 & 1.27 & 1.05 \\
7 & 0.3 & 0.76 & 0.70 & 0.80 & 0.59 \\
14 & 0.3 & 0.53 & 0.43 & 0.48 & 0.35 \\
28 & 0.3 & 0.26 & 0.24 & 0.27 & 0.19 \\
56 & 0.3 & 0.15 & 0.13 & 0.13 & 0.10 \\
1 & 0.5 & 1.53 & 1.45 & 1.58 & 1.37 \\
3 & 0.5 & 0.94 & 0.80 & 0.97 & 0.77 \\
7 & 0.5 & 0.55 & 0.53 & 0.58 & 0.43 \\
14 & 0.5 & 0.32 & 0.31 & 0.34 & 0.23 \\
28 & 0.5 & 0.18 & 0.18 & 0.19 & 0.14 \\
\hline
\end{tabular}

Table 5. Test I: DW'PF glass (crushed)-deionized water.

\begin{tabular}{cccccc}
\hline & \multicolumn{5}{c}{ Normalized elemental leach rate $\left(\mathrm{g} / \mathrm{m}^{2} \cdot \mathrm{d}\right)$} \\
\cline { 2 - 6 } Leach time & SA/V & \multicolumn{1}{c}{ Li } & B & Na & Si \\
\hline 3 & 0.3 & 1.13 & 1.14 & 1.18 & 0.93 \\
7 & 0.3 & 0.80 & 0.80 & 0.82 & 0.62 \\
14 & 0.3 & 0.47 & 0.47 & 0.48 & 0.36 \\
28 & 0.3 & 0.31 & 0.30 & 0.33 & 0.23 \\
56 & 0.3 & 0.16 & 0.16 & 0.16 & 0.12 \\
3 & 0.5 & 0.91 & 0.91 & 0.94 & 0.72 \\
7 & 0.5 & 0.57 & 0.57 & 0.59 & 0.44 \\
14 & 0.5 & 0.37 & 0.37 & 0.38 & 0.28 \\
28 & 0.5 & 0.21 & 0.21 & 0.22 & 0.16 \\
56 & 0.5 & 0.11 & 0.11 & 0.11 & 0.086 \\
1 & 1.0 & 1.19 & 1.17 & 1.26 & 0.93 \\
3 & 1.0 & 0.66 & 0.65 & 0.69 & 0.50 \\
7 & 1.0 & 0.39 & 0.37 & 0.40 & 0.29 \\
14 & 1.0 & 0.23 & 0.23 & 0.24 & 0.17 \\
28 & 1.0 & 0.14 & 0.14 & 0.15 & 0.10 \\
\hline
\end{tabular}

graphically in Figs. 4-7. In general, there is good agreement between the two forms of glass for all four elements. In examining the leach rate values in Tables 4 and 5, the lithium, boron, and sodium values agree very well for each particular leaching period, thus suggesting congruent dissolution of

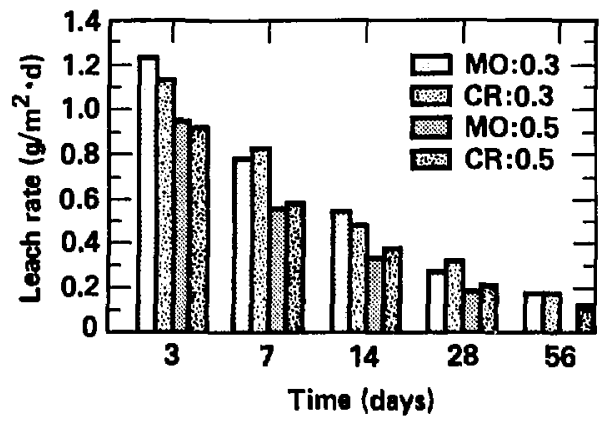

Figure 4. Lithium leach rate: monolith vs crushed glass.

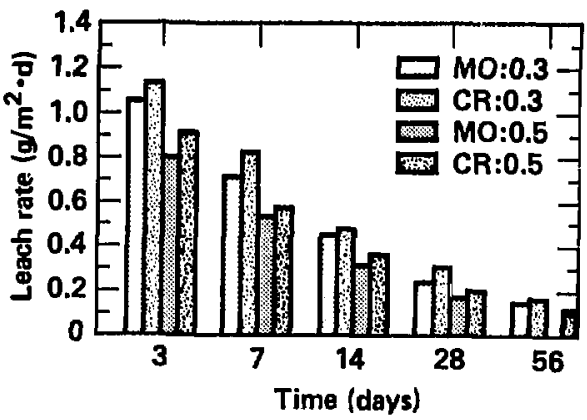

Figure 5. Boron leach rate: monolith vs crushed glass.

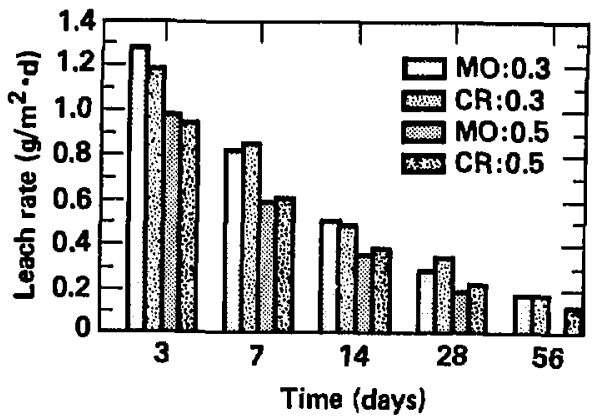

Figure 6. Sodium leach rate: monolith vs crushed glass.

the glass. However, the silicon values are correspondingly lower by as much as $30 \%$, suggesting perhaps that the leaching mechanism is affected by precipitation of insoluble phases or saturation of certain elements such as silicon. 


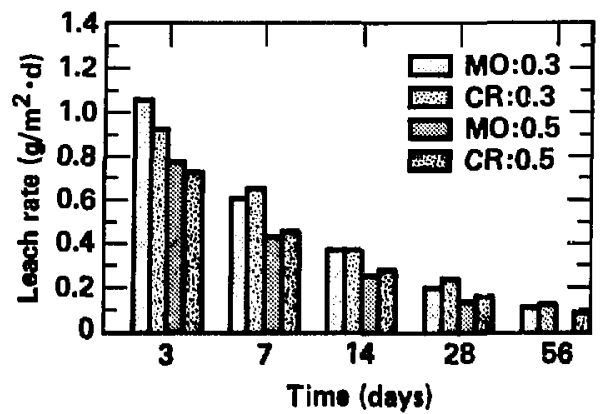

Figure 7. Silicon leach rate: monolith vs crushed glass.

We have also plotted the leach rate data for the crushed glass samples in Figs. 8-11. Each one of these plots represents a comparison of the three sets of data corresponding to SA/V ratios of 0.3 , 0.5 , and $1.0 \mathrm{~cm}^{-1}$. This comparison is possible when the SA/V ratios are multiplied by the leaching time. As seen in the graphs, the lithium, boron, sodium, and silicon data are all consistent and prove that, for the range studied of 0.3 to 1.0 $\mathrm{cm}^{-1}$, the leach rates are comparable regardless of the surface area of the sample and the volume of the leachant, so long as they are compared at the same value of $(\mathrm{SA} / \mathrm{V})(\mathrm{t})$.

As stated previously, Tests II, III, and IV were different from Test I in that J-13 water was the leachant. Furthermore, Tests III and IV were complicated by using crushed tuff in Test III and crushed tuff and stainless steel in Test IV. The data from these tests appear in Appendices C, D, and E. J-13 is a tuffaceous formation ground water that contains $0.14,27$, and $42 \mathrm{ppm}$ of boron, silicon, and sodium, respectively, based on the average of three separate analyses (unequilibrated $\mathrm{J}-13$ ), as shown in Table 3. Before Tests II, III, and IV were conducted, the J-13 water was equilibrated with powdered tuff (pretreated tuff at a ratio of $20 \mathrm{ml}$ to $1 \mathrm{~g}$ of tuff sor 30 days at $90^{\circ} \mathrm{C}$. During this equilibration time, the elemental concentrations in J-13 changed as shown in Table 3; based on the average of three separate analyses, the boron, silicon, and sodium concentrations increased to $0.22,39$, and $47 \mathrm{ppm}$, respectively. In examining the ICP data from Tests II, III, and IV, we decided that the boron, silicon, and sodium data were not suitable for calculation of leach rates because, in most instances, the data for the real samples and for the blank samples were of the same magnitude. In a few cases, the blanks

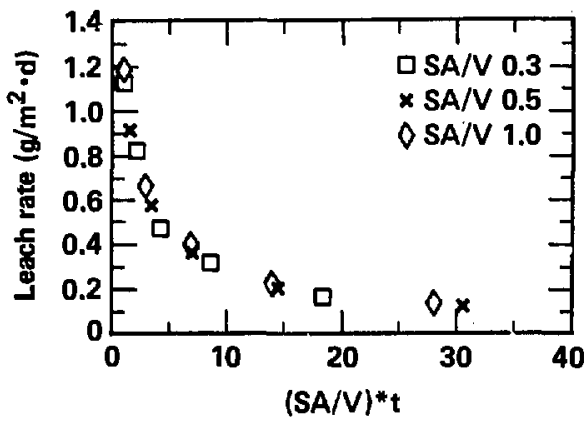

Figure 8. SA/V test: lithium.

were slightly higher than the samples but this artifact may be attributable to statistical variations in the measurements.

In view of the above circumstances surrounding the boron, sodium, and silicon data, only the lithium data from Tests II, III, and IV appear to be useful in assessing the durability of the DWPF glass from test to test. We have tabulated in Table 6 the calculated leach rates for lithium for all tests at SA/V ratios of 0.3 and $0.5 \mathrm{~cm}^{-1}$. The data columns are ordered according to the complexity of the tests to show differences in the values from test to test. First, it appears that glass behavior depends very much on the type of leachant since the leach rates in deionized water are about one order of magnitude higher than in J-13 water. Second, it is not clear that the additions of tuff in Test III and tuff and stainless steel in Test IV have any effect on the lithium leach rates; these appear to be about the same as in Test II with the exception of the day-3 samples. The data for the day- 28 and day- 41 samples are shown

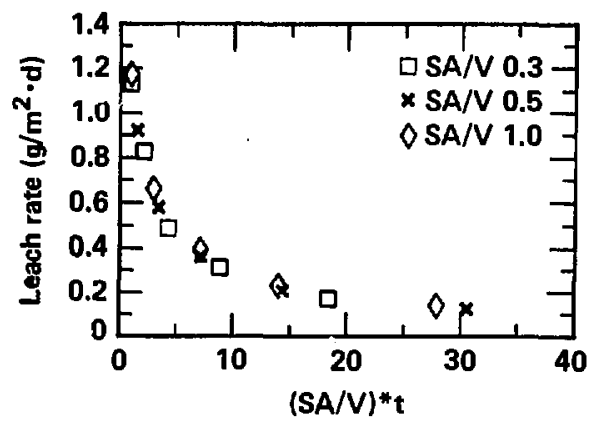

Figure 9. SA/V test: boron. 


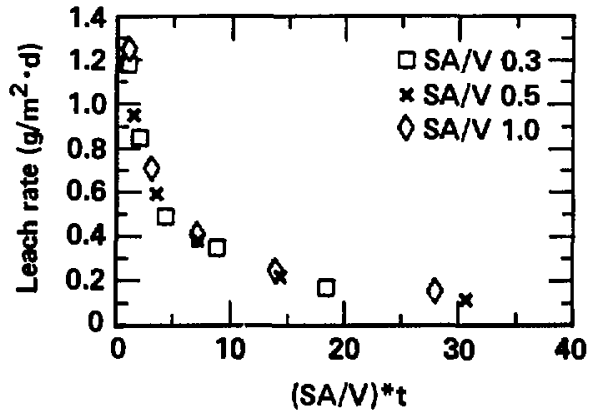

Figure 10. SA/V test: sodlum.

with an uncertainty that represents the standard deviation of the average of two separate samples.

The lithium leach rates from Test I (deionized water) and from Test II (J-13 water) for monolith samples at $S A / V$ ratios of 0.3 and $0.5 \mathrm{~cm}^{-1}$ have been plotted in Fig. 12 to show the comparison of the $(S A / V)(t)$ relationship in deionized water and J-13 water. Aside from the fact that the J-13 leach rates are about an order of magnitude lower, the $(S A / V)(t)$ relationship in J-13 water appears to be somewhat consistent with deionized water.

A summary of the $\mathrm{pH}$ data obtained in Tests 1, II, III, and IV is presented in Table 7. These are final $\mathrm{pH}$ values only for the leachates from samples run at SA/V ratios of 0.3 and $0.5 \mathrm{~cm}^{-1}$. There is no clear trend in the $\mathrm{pH}$ as a function of the complexity test in the $0.3-\mathrm{cm}^{-1}$ samples, and only a slight decreasing trend is present in the $0.5-\mathrm{cm}^{-1}$ samples. As a function of reaction time, the $\mathrm{pH}$ readings appear to increase slightly in both the $0.3-$ and $0.5-\mathrm{cm}^{-1}$ samples.

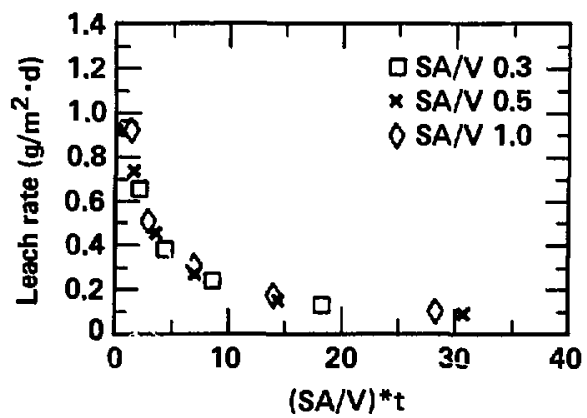

Figure 11. SA/V test: silicon.
Table 6. Normalized elemental leach rate $\left(\mathrm{g} / \mathrm{m}^{2} \cdot \mathrm{d}\right)$.

\begin{tabular}{|c|c|c|c|c|c|c|c|}
\hline \multirow{3}{*}{$\begin{array}{l}\text { Leach } \\
\text { time }\end{array}$} & \multicolumn{7}{|c|}{ Lithium } \\
\hline & \multicolumn{4}{|c|}{$S A / V=0.3 \mathrm{~cm}^{-1}$} & \multicolumn{3}{|c|}{$S A / V=0.5 \mathrm{~cm}^{-1}$} \\
\hline & $\mathbf{T}$ & III & III & IV & $\mathbf{I}$ & II & III IV \\
\hline 3d & 1.22 & 0.0075 & 一 & 0.035 & 0.094 & 0.15 & -0.066 \\
\hline $7 d$ & 0.76 & 0.045 & 0.047 & 0.042 & 0.55 & 0.043 & -0.044 \\
\hline 14d & 0.53 & 0.031 & 0.038 & 0.037 & 0.32 & 0.022 & -0.042 \\
\hline 28d & $\begin{array}{l}0.26 \\
\pm 5 \%\end{array}$ & $\begin{array}{r}0.023 \\
\pm 3 \%\end{array}$ & $\begin{array}{l}0.019 \\
\pm 22 \%\end{array}$ & 0.023 & $\begin{array}{l}0.18 \\
\pm 4 \%\end{array}$ & $\begin{array}{l}0.018 \\
\pm 70 \%\end{array}$ & $\begin{array}{r}-0.024 \\
\pm 12 \%\end{array}$ \\
\hline $56 d$ & $\begin{array}{l}0.15 \\
\pm 1 \%\end{array}$ & 0.022 & Lout & 0.011 & 一 & 0.018 & -0.015 \\
\hline 91d & 一 & - & $\begin{array}{l}0.012 \\
\pm 7 \%\end{array}$ & $\begin{array}{l}0.010 \\
\pm 3 \%\end{array}$ & - & - & $\begin{array}{r}-0.010 \\
\pm 8 \%\end{array}$ \\
\hline $82 \mathrm{~d}$ & - & - & 9.008 & - & & & \\
\hline $\begin{array}{r}\text { I } \\
\text { II } \\
\text { III } \\
\text { IV }\end{array}$ & $\begin{array}{l}=\mathbf{G l} \\
=\mathbf{G l} \\
=\mathbf{G l} \\
=\mathbf{G l}\end{array}$ & $\begin{array}{r}195+D \\
195+J \\
\text { ass }+J- \\
295+J-\end{array}$ & $\begin{array}{l}\text { I water } \\
13 \text { wate } \\
13 \text { wate } \\
13 \text { wate }\end{array}$ & $\begin{array}{l}\mathbf{r}+ \\
\mathbf{r}+\end{array}$ & & & ceel \\
\hline
\end{tabular}

Pertaining to mass loss of glass specimens during the leaching interval, we note that the mass loss for most samples is very small, usually less than $0.01 \%$, so that conclusions based on mass loss are not possible. Pertaining to weight loss of the leachate during the same leaching intervals, we note that the losses for most leachates are on the order of a few percent (less than 10\%). In a few cases, however, and particularly in the

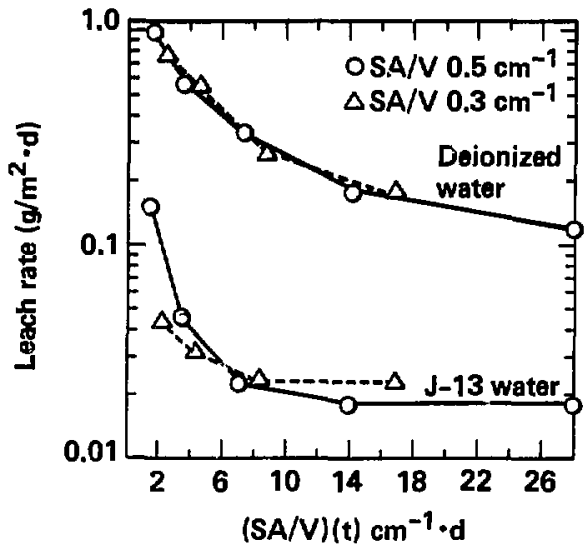

Figure 12. Comparison of deionized water and J-13 water (SA/V)(t) vs lithium leach rate. 
Table 7. Summary of $\mathbf{p H}$ data in the leachates.

\begin{tabular}{|c|c|c|c|c|c|c|c|c|}
\hline \multirow{2}{*}{$\begin{array}{l}\text { Leach } \\
\text { time }\end{array}$} & \multicolumn{4}{|c|}{$\mathrm{SA} / \mathrm{V}=0.3 \mathrm{~cm}^{-1}$} & \multicolumn{4}{|c|}{$S A / V=0.5 \mathrm{~cm}^{-1}$} \\
\hline & 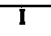 & III & III & IV & I & II & III & IV \\
\hline 3d & 8.20 & 9.17 & - & 9.37 & 9.73 & 9.21 & - & 8.92 \\
\hline $7 d$ & 9.25 & 9.21 & 9.08 & 9.45 & 9.76 & 9.32 & - & 9.29 \\
\hline $14 d$ & 9.52 & 9.18 & $\mathbf{9 . 3 7}$ & 9.54 & 9.90 & 9.44 & - & 9.18 \\
\hline $28 d$ & 9.74 & 9.45 & 9.35 & $\mathbf{9 . 6 3}$ & 9.80 & 9.50 & - & 9.62 \\
\hline $56 d$ & 9.73 & 9.43 & 9.55 & 9.45 & 9.84 & $\mathbf{9 . 5 1}$ & - & 9.62 \\
\hline 91d & - & - & 9.28 & 9.45 & - & - & - & 9.45 \\
\hline 182d & - & - & 9.30 & - & - & - & - & - \\
\hline $\begin{array}{r}\text { I } \\
\text { III } \\
\text { IV }\end{array}$ & $\begin{array}{l}+\mathrm{D} 1 \\
+\mathrm{J}-1 \\
+\mathrm{J}-1\end{array}$ & $\begin{array}{l}\text { tuff } \\
\text { luff }\end{array}$ & & & & & & \\
\hline
\end{tabular}

28-, 56-, and 91-day tests, we experienced losses from 10 to $64 \%$. The data from these tests should be considered suspect, and we did not use them for any calculations. We attribute these large losses to a defective leaching capsule or to the presence of tuff fines around the screw caps of the leaching capsules in the case of iests III and IV.

\section{Acknowledgments}

The ICP measurements were performed by $A$. Langhorst and $W$. Burks. We wish to thark them for their dedication. We also wish to acknowledge V. Oversby and R. Aines for reviewing this inat uscript. Their comments were certainly helpful.

\section{References}

Knauss, K. G. (1984), Petrologic and Geochemical Characterizniton of the Topopah Spring Member of the Paintbrush Tuff: Outcrop Samples used in Waste Package experiments, Lawrence Livermore National Laboratory, Livermore, CA, UCRL,-53558.

Oversioy, V. M. (1984), Reaction of the Topopah Spring Tuff with J-13 Well Water at $90^{\circ} \mathrm{C}$ and $150^{\circ} \mathrm{C}$, Lawrence Livermore National Laboratory, Livermore, CA, UCRL.-53552.

Bibler, N. E. (1983), Savannah River Laboratory, Aiken, SC, private communication, June 6, 1983.

Nuclear Waste Materials Handbook Test Methods, DOE/TIC-11400 (1983). 


\section{Appendix A. \\ Test I: DWPF Glass-Deionized Water Experiment, Monolith Examples}


TEST - I

DEIONIZED WATER EXPERIMENT

DWEF GLASS (MMOLITH)

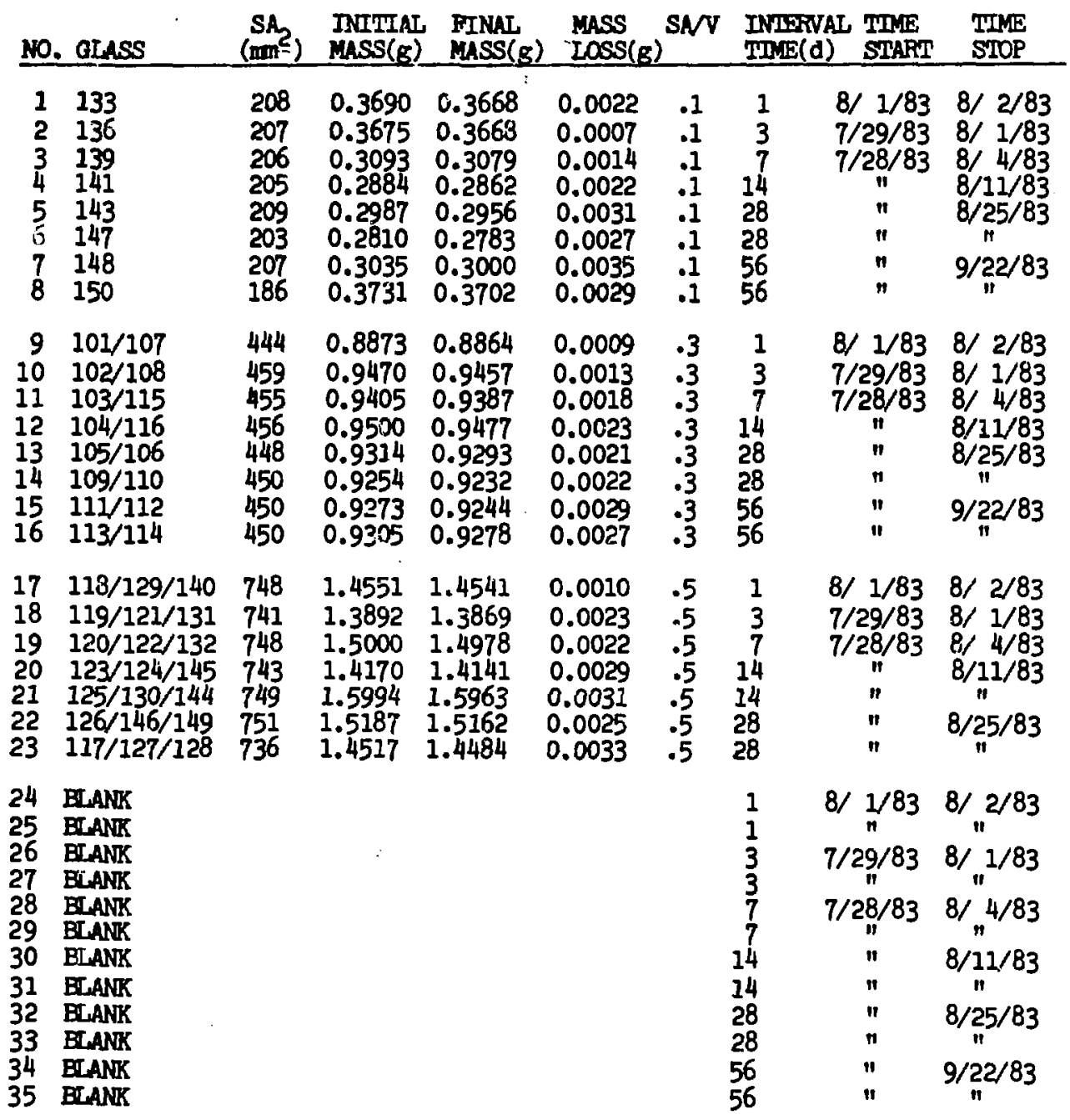


TEST - I

DEIONIZED WATER EXPERTEENT

DWPF GLASS (MONOLITH)

\begin{tabular}{|c|c|c|c|c|c|}
\hline \multirow[b]{2}{*}{ NO. } & \multirow[b]{2}{*}{ WATER(g) } & \multirow{2}{*}{$\begin{array}{l}\text { INITIAL } \\
\text { WT. ( } 8 \text { ) }\end{array}$} & \multirow{2}{*}{$\begin{array}{l}\text { FINAL } \\
\text { Wr. }(\mathrm{g})\end{array}$} & \multicolumn{2}{|c|}{ LEACHANI } \\
\hline & & & & $\operatorname{Loss}(\mathrm{g})$ & $\operatorname{Lass}(x)$ \\
\hline $\begin{array}{l}1 \\
2 \\
3 \\
4 \\
5 \\
6 \\
7 \\
8\end{array}$ & $\begin{array}{l}20.8 \\
20.7 \\
20.6 \\
20.5 \\
20.9 \\
20.3 \\
20.7 \\
18.6\end{array}$ & $\begin{array}{l}104.55 \\
103.94 \\
104.52 \\
104.24 \\
104.55 \\
103.94 \\
104.31 \\
102.18\end{array}$ & $\begin{array}{l}104.48 \\
103.83 \\
104.35 \\
103.94 \\
103.91 \\
103.38 \\
102.31 \\
100.74\end{array}$ & $\begin{array}{l}0.07 \\
0.11 \\
0.17 \\
0.30 \\
0.64 \\
0.56 \\
2.00 \\
1.44\end{array}$ & $\begin{array}{l}0.34 \% \\
0.53 \% \\
0.83 \% \\
1.87 \% \\
3.71 \% \\
2.11 \% \\
9.11 \% \\
7.74 \%\end{array}$ \\
\hline $\begin{array}{l}9 \\
10 \\
11 \\
12 \\
13 \\
14 \\
15 \\
16\end{array}$ & $\begin{array}{l}14.8 \\
15.3 \\
15.2 \\
15.2 \\
14.9 \\
15.0 \\
15.0 \\
15.0\end{array}$ & $\begin{array}{r}98.96 \\
100.08 \\
98.90 \\
98.65 \\
99.01 \\
99.51 \\
99.17 \\
98.73\end{array}$ & $\begin{array}{l}98.88 \\
99.95 \\
98.63 \\
98.31 \\
98.53 \\
98.94 \\
98.10 \\
97.34\end{array}$ & $\begin{array}{l}0.08 \\
0.13 \\
0.27 \\
0.34 \\
0.48 \\
0.57 \\
1.07 \\
1.39\end{array}$ & $\begin{array}{l}0.54 \% \\
0.85 \% \\
1.78 \% \\
2.24 \% \\
3.22 \% \\
3.80 \% \\
7.13 \% \\
9.27 \%\end{array}$ \\
\hline $\begin{array}{l}17 \\
18 \\
19 \\
20 \\
21 \\
22 \\
23\end{array}$ & $\begin{array}{l}15.0 \\
14.8 \\
15.0 \\
14.9 \\
15.0 \\
15.0 \\
14.7\end{array}$ & $\begin{array}{l}99.85 \\
98.87 \\
99.08 \\
98.95 \\
99.86 \\
99.95 \\
99.77\end{array}$ & $\begin{array}{l}99.73 \\
98.77 \\
98.89 \\
98.37 \\
99.55 \\
99.40 \\
99.23\end{array}$ & $\begin{array}{l}0.12 \\
0.10 \\
0.19 \\
0.58 \\
0.31 \\
0.55 \\
0.54\end{array}$ & $\begin{array}{l}0.80 \pi \\
0.68 \% \\
1.27 \pi \\
3.89 \pi \\
2.07 \% \\
3.67 \% \\
3.67 \%\end{array}$ \\
\hline $\begin{array}{l}24 \\
25 \\
26 \\
27 \\
28 \\
29 \\
30 \\
31 \\
32 \\
33 \\
34 \\
35\end{array}$ & $\begin{array}{l}17.0 \\
17.0 \\
17.0 \\
17.0 \\
17.0 \\
17.0 \\
17.0 \\
17.0 \\
17.0 \\
17.0 \\
17.0 \\
17.0\end{array}$ & $\begin{array}{r}100.14 \\
99.86 \\
100.37 \\
99.77 \\
99.73 \\
99.67 \\
99.58 \\
100.30 \\
99.79 \\
99.59 \\
100.15 \\
100.33\end{array}$ & $\begin{array}{r}100.05 \\
99.79 \\
100.26 \\
99.67 \\
99.56 \\
99.44 \\
98.97 \\
99.86 \\
99.73 \\
99.13 \\
99.01 \\
99.31\end{array}$ & $\begin{array}{l}0.09 \\
0.07 \\
0.11 \\
0.10 \\
0.17 \\
0.23 \\
0.61 \\
0.44 \\
0.06 \\
0.46 \\
1.14 \\
1.02\end{array}$ & $\begin{array}{l}0.53 \% \\
0.41 \% \\
0.65 \% \\
0.59 \% \\
1.00 \% \\
1.35 \% \\
3.59 \% \\
2.59 \% \\
0.35 \% \\
2.71 \% \\
6.71 \% \\
6.00 \%\end{array}$ \\
\hline
\end{tabular}




\section{TEST I}

DEIONIZED WATER EXPERIMENT

DUPF GLASS (MOMOLITH)

DY 1 - ICP Analys Is

\section{SNPLES}

\begin{tabular}{lll}
$S A / Y=0.1$ & 0.3 & 0.5 \\
\hline
\end{tabular}

BANKS

ELEM DET. LIM.

$9 \quad 17$

24

25

$\begin{array}{lllllll}\text { Al } & .008 & .266 & 1.19 & 1.78 & <.008 & .014 \\ \text { B } & .004 & .195 & .091 & 1.64 & <.004 & <.004 \\ \mathrm{Fe} & .002 & .040 & .150 & .330 & .003 & .007 \\ \mathrm{Li} & .001 & .225 & 1.15 & 1.81 & .001 & .001 \\ \mathrm{Mn} & .0003 & .047 & .071 & .114 & <.0003 & <.0003 \\ \mathrm{HI} & .008 & .008 & .015 & .023 & <.008 & <.008 \\ \mathrm{St} & .004 & 2.45 & 11.1 & 17.4 & .092 & .088 \\ \mathrm{Sr} & .012 & .012 & .030 & .038 & <.012 & <.012 \\ \mathrm{Zr} & .002 & .004 & .004 & .007 & <.002 & <.002 \\ \mathrm{C} & .004 & .074 & .398 & .448 & <.004 & <.004 \\ \mathrm{R} & .260 & .264 & <.260 & <.260 & <.260 & <.260 \\ \mathrm{Mg} & .0004 & .126 & .351 & .040 & .014 & .034 \\ \mathrm{Na} & .004 & .891 & 4.34 & 6.81 & .047 & .034\end{array}$

Note: Detection 1 init is 4 times the standard deviation of the blank.

Results are in micrograns per allifliter (or pm). 
TEST I

DEIONIZED MATER EXPERINENT

DUPF GLASS (mOMOLITH)

DAY 3 - ICP Analys Is

SNPLES

\begin{tabular}{lll}
$S N V=0.1 \quad 0.3$ & 0.5 \\
\hline
\end{tabular}

BLAMKS

ELEM DET. LIM.

10

18

26

27

$\begin{array}{lllllll}\text { Al } & .008 & 1.21 & 2.28 & 2.72 & .019 & .090 \\ \mathrm{~B} & .004 & .999 & 2.13 & 2.71 & <.004 & <.004 \\ \mathrm{Fe} & .002 & .079 & .357 & .469 & .011 & .006 \\ \mathrm{LI} & .001 & 1.12 & 2.59 & 3.32 & .001 & <.001 \\ \mathrm{Mn} & .0003 & .031 & .132 & .174 & <.0003 & <.0003 \\ \mathrm{Ni} & .003 & .010 & .025 & .036 & .002 & .002 \\ \mathrm{Si} & .004 & 11.2 & 24.0 & 29.3 & .110 & .124 \\ \mathrm{Sr} & .012 & .031 & .032 & .022 & .003 & .003 \\ \mathrm{Zr} & .002 & .004 & .006 & .010 & <.002 & <.002 \\ \mathrm{Ca} & .004 & .374 & .402 & .339 & <.004 & <.004 \\ \mathrm{~K} & .260 & <.260 & .398 & <.260 & <.260 & <.260 \\ \mathrm{Hg} & .0004 & .203 & .069 & .070 & .040 & .029 \\ \mathrm{Na} & .004 & 4.24 & 9.74 & 12.4 & .046 & .026\end{array}$




\title{
TEST I
}

\section{DEIONIZED MATER EXPERIMENT}

\author{
DUPF GLASS (MONOLITH)
}

DAY 7 - ICP Analys is

\section{SA/V=0.1}

ELEM DET. LIM.

\begin{tabular}{|c|c|c|c|c|c|c|}
\hline A) & .008 & 1.66 & 2.95 & 3.47 & .021 & .005 \\
\hline B & .004 & 1.60 & 3.39 & 4.22 & $<.004$ & ২.004 \\
\hline Fe & .002 & .135 & .410 & .539 & .013 & .011 \\
\hline 41 & .001 & 1.77 & 3.82 & 4.57 & $<.001$ & S.001 \\
\hline Mn & .0003 & .085 & .134 & .145 & $<.0003$ & $<.0003$ \\
\hline$M i$ & .008 & .017 & .028 & .031 & R.008 & R.008 \\
\hline St & .004 & 16.6 & 32.2 & 38.8 & .097 & .104 \\
\hline $\mathbf{S r}$ & .012 & .026 & .010 & .008 & $<.012$ & $<.012$ \\
\hline $2 r$ & .002 & .002 & .004 & .015 & $<.002$ & $<.002$ \\
\hline Ca & .004 & .410 & .296 & .189 & $<.004$ & $<.004$ \\
\hline $\mathbf{K}$ & .260 & $<.260$ & $<.260$ & $<.260$ & $<.260$ & $<.260$ \\
\hline$M g$ & .0004 & .134 & .162 & .074 & .048 & .030 \\
\hline $\mathrm{Na}$ & .004 & 6.80 & 14.6 & 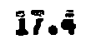 & 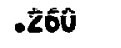 & $<.004$ \\
\hline
\end{tabular}


TEST I

DEIOHIZED WATER EXPERIFENT

DWPF GLASS (MOMOLITH)

DAY 14 - ICP Analysis

SAMLES

$S N / V=0.1$

0.3

0.5

0.5

BLANKS

ELEM DET. LIM.

12

20

21

30

A1 $\quad .008$

2.17

3.50

3.85

3.97

$.017 \quad .013$

B $\quad .004$

2.34

4.26

4.98

5.05

$<.004<.004$

Fe $\quad .002$

$.226 \quad .639$

.885

1.06

$.012 \quad .012$

Lf .001

2.62

5.37

5.37

5.55

$<.001<.001$

Mn $\quad .0003$

.077

.222

.222

.246

$<.0003<.0003$

$\mathrm{Ni} \quad .008$

.015

.033

.039

.042

$<.008$

$<.008$

23.0

39.1

44.4

45.3

.221

.118

.022

.018

.014

.016

$<.012<.012$

.005

.017

.035

.041

$<.002<.002$

.383

.219

.184

.220

$<.004<.004$

$<.260$

.302

.271

.406

$<.260$

$<.260$

.059

.074

.081

.098

10.1

17.7

20.6

21.2

.033

.038

$.008 \quad .004$ 


\section{TEST I}

\section{DEIONIZED HATER EXPERIMENT}

DHPF GLASS (MONOLITH)

$$
\text { DAY } 28 \text { - ICP Analysis }
$$

\section{SAPLES}

$\begin{array}{llllllll}S A & V=0.1 & 0.1 & 0.3 & 0.3 & 0.5 & 0.5 & \text { BLANKS }\end{array}$

\section{ELEM DET. LIM.}

\begin{abstract}
A
\end{abstract}
B

Fe

L

H

Ni

Si

Sr

Zr

C.

K

$\mathrm{Mg}$

$\mathrm{Na}$

$$
.012
$$$$
.260
$$

$$
.008
$$

$.004 \quad 3.20$

$\begin{array}{lll}.004 & 3.20 & 3.07\end{array}$

$\begin{array}{lll}.002 & .239 & .334\end{array}$

$.001 \quad 3.51 \quad 3.26$

$.0003 \quad .091 \quad .05$

$.008 \quad .019$

000430.0

.019

28.7$$
.019
$$$$
.024
$$$$
.002 \quad .007
$$$$
.018
$$$$
\begin{array}{lll}
.004 & .293 & .349
\end{array}
$$$$
.268
$$$$
<.260
$$

$\begin{array}{lll}.0004 & .042 \quad .065\end{array}$

$.004 \quad 13.6 \quad 12.5$
13

14

22

23

32

33

$\begin{array}{llllllllll}\mathrm{Mg} & .0004 & .042 & .065 & \mathrm{M} & \mathrm{M} & .190 & .159 & .021 & .034 \\ \mathrm{Na} & .004 & 13.6 & 12.5 & 19.1 & 20.0 & 23.1 & 22.6 & .008 & .011\end{array}$

Note: $M$ - elemental analysis not avallable. 


\section{TEST I}

DEIONIZED WATER EXPERIRENT

DWPF GLASS (MOHOLITH)

DAY 56 - ICP Analysis

SAPLES

$\begin{array}{llll}S A / V=0.1 & 0.1 & 0.3 & 0.3\end{array}$

BLANKS

\begin{tabular}{|c|c|c|c|c|c|c|c|}
\hline ELEM & DET. LIM. & 7 & 8 & 15 & 16 & 34 & 35 \\
\hline$A 1$ & .008 & 2.89 & 2.85 & 3.90 & 3.92 & .210 & .210 \\
\hline B & .004 & 3.78 & 3.73 & 5.67 & 5.72 & $<.004$ & <.004 \\
\hline Fe & .002 & .364 & .475 & 1.83 & 1.85 & .008 & .015 \\
\hline LI & .001 & 4.14 & 4.26 & 6.49 & 6.45 & .001 & .001 \\
\hline$M n$ & .0003 & .142 & .158 & .441 & .457 & $<.001$ & $<.001$ \\
\hline N1 & .008 & .021 & .022 & .048 & .047 & $<.010$ & $<.010$ \\
\hline Si & .004 & 35.1 & 37.8 & 49.1 & 49.5 & .140 & .250 \\
\hline $\mathbf{S r}$ & .012 & .026 & .020 & .023 & .028 & $<.010$ & $<.010$ \\
\hline $\mathbf{Z r}$ & .002 & .035 & .038 & .034 & .035 & $<.010$ & $<.010$ \\
\hline $\mathrm{Ca}$ & .004 & .243 & .253 & .299 & .299 & $<.010$ & $<.010$ \\
\hline : & .260 & $<.260$ & $<.260$ & $<.260$ & $<.260$ & $<.260$ & $<.260$ \\
\hline $\mathrm{Mg}$ & .0004 & M & MR & MA & $M$ & MA & MA \\
\hline $\mathrm{Ma}$ & .004 & 15.2 & 15.7 & 23.8 & 23.6 & .010 & .010 \\
\hline
\end{tabular}

Hote: KA - elemental analysis not avaflable. 


\section{TEST I}

DEIOHIZED WATER EXPERIMENT

DWPF GLASS (MONOLITH)

RAH DATA TABULATION - LEACHATE NALYSIS

DAY 1

Analytical Results

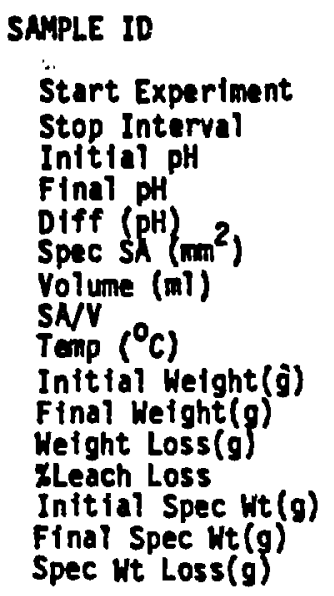

SAMPLE ID

Start Experiment

Stop Interval

Initiai pH

Spec SA $\left(\mathrm{mm}^{2}\right)$

Volume (mi)

Temp $\left({ }^{\circ} \mathrm{C}\right)$

Initial Weight $(\hat{g})$

Final Height $(g)$

XLeach Loss

Initial Spec Wt(g)

Spec Wt Loss(g)
1

$8 / 1 / 83$

$8 / 2 / 83$

5.4

5.5

0.1

208.

20.8

0.1

90.0

104.55

104.48

0.07

0.348

0.3690

0.3668

0.0022
9

$8 / 1 / 83 \quad 8 / 2 / 83$

$8 / 2 / 83 \quad 8 / 2 / 83$

5.4

5.6

0.2

44.

14.8

0.3

90.0

98.96

98.88

0.08

$0.54 \%$

0.8873

0.8864

0.0009
5.4

5.9

0.5

748.

15.0

0.5

90.0

99.85

99.73

0.12

$0.08 \%$

1.4551

1.4541

0.0010

\section{CONSTITUENT ANALYSIS(mg/l1ter)}

Analytical Result

$\begin{array}{ll}9 & 17 \\ 1.19 & 1.78 \\ 0.991 & 1.64 \\ 0.150 & 0.330 \\ 1.15 & 1.81 \\ 0.071 & 0.114 \\ 0.015 & 0.023 \\ 11.1 & 17.4 \\ 0.030 & 0.038 \\ 0.004 & 0.007 \\ 0.398 & 0.448 \\ <.260 & 5.260 \\ 0.351 & 0.040 \\ 4.34 & 15.81\end{array}$

Corrected for Blank

$\begin{array}{lll}\text { Cl } & C 9 & \mathrm{Cl}\end{array}$

$\begin{array}{lll}0.266 & 1.19 & 1.78 \\ 0.195 & 0.991 & 1.64 \\ 0.035 & 0.145 & 0.325 \\ 0.224 & 1.14 & 1.80 \\ 0.047 & 0.071 & 0.114 \\ 0.008 & 0.015 & 0.023 \\ 2.36 & 11.0 & 17.3 \\ 0.012 & 0.030 & 0.038 \\ 0.004 & 0.004 & 0.007 \\ 0.074 & 0.398 & 0.448 \\ 0.264 & 1.260 & <.260 \\ 0.102 & 0.327 & 0.016 \\ 0.85 & 4.30 & 6.77\end{array}$




\section{TEST I}

\section{DEIONIZED MATER EXPERIMENT}

DUPF GLASS (MONOLITH)

\section{RAW DATA TABULATION - LEACHATE AHLYSIS \\ DAY 3}

Analytical Result

\section{SAPLE ID}

Start Experiment

Stop Interval

Initial pH

Finel pH

Diff (pH) ${ }_{\text {Spec }}^{2} \AA^{2}$ )

Volume (ni)

SA/N

Tenp $\left({ }^{\circ} \mathrm{C}\right)$

Inftial Height(g)

Final Weight $(g)$

Weight Loss $(g)$

Xleach Loss

Initial Spec wt $(g)$

Final Spec Wt $(g)$

Spec Wt Loss(g)

2

2

10

18

$\begin{array}{lll}7 / 29 / 83 & 7 / 29 / 83 & 7 / 29 / 83 \\ 8 / 1 / 83 & 8 / 1 / 83 & 8 / 1 / 83 \\ 5.4 & 5.4 & 5.4 \\ 5.9 & 6.6 & 6.6 \\ 0.5 & 1.2 & 1.2 \\ 207 . & 459 . & 741 . \\ 20.7 & 15.3 & 14.8 \\ 0.1 & 0.3 & 0.5 \\ 90.0 & 90.0 & 90.0 \\ 103.94 & 100.08 & 98.87 \\ 103.83 & 99.95 & 98.77 \\ 0.11 & 0.13 & 0.10 \\ 0.53 \% & 0.85 \% & 0.68 \% \\ 0.3675 & 0.9470 & 1.3892 \\ 0.3668 & 0.9457 & 1.3869 \\ 0.0007 & 0.0013 & 0.0023\end{array}$

\section{CONSTITUENT ANRLYSIS(mg// ItER)}

Analytical Result

Corrected for Blank

SAMPLE ID

2

10

18

C2

C10

C18

\begin{tabular}{|c|c|c|c|c|c|c|}
\hline $\begin{array}{l}A 1 \\
B \\
\text { Fe } \\
\text { Li } \\
M n \\
M_{1} \\
S i \\
S r\end{array}$ & $\begin{array}{l}1.21 \\
0.999 \\
0.079 \\
1.12 \\
0.033 \\
0.010 \\
11.2 \\
0.031 \\
0.004 \\
0.374 \\
8.260 \\
0.203 \\
4.21\end{array}$ & $\begin{array}{l}2.28 \\
2.13 \\
0.357 \\
2.59 \\
0.132 \\
0.025 \\
24.0 \\
0.032 \\
0.006 \\
0.402 \\
.398 \\
0.064 \\
9.74\end{array}$ & $\begin{array}{l}2.72 \\
2.71 \\
0.469 \\
3.32 \\
0.174 \\
0.036 \\
29.3 \\
0.022 \\
0.010 \\
0.334 \\
2.260 \\
0.070 \\
12.4\end{array}$ & $\begin{array}{l}1.16 \\
0.999 \\
0.071 \\
1.12 \\
0.031 \\
0.080 \\
11.1 \\
0.028 \\
0.004 \\
0.374 \\
<.260 \\
0.169 \\
4.20\end{array}$ & $\begin{array}{l}2.22 \\
2.13 \\
0.349 \\
2.59 \\
0.132 \\
0.023 \\
23.9 \\
0.029 \\
0.006 \\
0.402 \\
.398 \\
0.035 \\
9.70\end{array}$ & $\begin{array}{l}2.67 \\
2.71 \\
0.461 \\
3.32 \\
0.174 \\
0.034 \\
29.2 \\
0.019 \\
0.010 \\
0.339 \\
<.260 \\
0.036 \\
12.0\end{array}$ \\
\hline
\end{tabular}


TEST I

DEIONIZED WATER EXPERIMENT

DHPF GLASS (MONOLITH)

RAH DATA TABULATION - LEACHATE AWLYSIS

DAY 7

Analytical Results

SNPLE ID

3

11

19

Start Experiment

Stop Interval

Inftlal pH

Final $\mathrm{PH}$

Diff $(\mathrm{pH})$
Spec $\mathrm{SA}\left(\mathrm{m} \mathrm{m}^{2}\right)$

volume $(g)$

SNY

Temp $\left({ }^{\circ} \mathrm{C}\right)$

Initlai Helght $(g)$

Final Weight $(g)$

Weight Loss(g)

KLeach Loss

Initlar Spec Wt(g)

Final Spec it $(g)$

Spec Wt Loss (g)

$\begin{array}{lll}7 / 28 / 83 & 7 / 28 / 83 & 7 / 28 / 83 \\ 8 / 4 / 83 & 8 / 4 / 83 & 8 / 4 / 83 \\ 5.4 & 5.4 & 5.4 \\ 5.9 & 6.2 & 6.6 \\ 0.5 & 0.8 & 1.2 \\ 206 . & 455 . & 748 . \\ 20.6 & 15.2 & 15.0 \\ 0.1 & 0.3 & 0.5 \\ 90.0 & 90.0 & 90.0 \\ 104.52 & 98.90 & 99.08 \\ 104.35 & 98.63 & 98.89 \\ 0.17 & 0.27 & 0.19 \\ 0.834 & 1.787 & 1.278 \\ 0.3093 & 0.9405 & 1.5000 \\ 0.3079 & 0.9387 & 1.4978 \\ 0.0014 & 0.0018 & 0.0022\end{array}$

CONSTITUERT AMALYSIS(mg/liter)

Analytical Result

Corrected for Blank

SAMPLE ID

3

11

19

C3

C11

C19

$\begin{array}{ll}\text { Al } & 1.66 \\ \text { B } & 1.60 \\ \text { fe } & 0.135 \\ \text { LI } & 1.77 \\ \text { Wn } & 0.085 \\ \text { Mi. } & 0.017 \\ \text { Si } & 16.6 \\ \text { Sr } & 0.026 \\ \text { Zr } & 0.002 \\ \text { Ca } & 0.410 \\ \text { K } & <.260 \\ \text { Mg } & 0.134 \\ \text { Ha } & 6.80\end{array}$

2.95

3.39

0.410

3.82

0.134

0.028

32.2

0.010

3.47

1.65

2.94

3.46

4.22

0.539

4.57

0.145

0.031

38.8

0.008

1.60

0.123

3.39

1.77

0.398

0.085

0.017

3.82

0.134

16.5

0.028

32.1

0.026

0.010

0.002

0.004

0.110

0.296

$<.260$

<.260

0.095

0.123

4.22

0.296

0.189

0.162

0.074

6.54

14.3

0.527

4.57

0.145

0.031

38.7

0.008

0.015

0.189

$<.260$

17.4

0.035

17.1 


\section{TEST I}

DEIONIZEO WATER EXPERIMENT

DUPF GLASS (MONOLITH)

RAH DATA TABULATION - LEACHATE AHLYSIS

DAY 14

Anelytical Results

SAPLE ID

Start Experiment

Stop Intervul

Initial pH

Final pH

Diff (pH) ${ }^{2}$ )

Yolume (g)

SAN

Temp $\left({ }^{\circ} \mathrm{C}\right)$

Init lal Welght (g)

Final Wefght $(g)$

Welght Loss(g)

Xleach Loss

Initfal Spec Wt $(g)$

Final Spec Wt $(g)$

Spec ut Loss $(g)$
4

$7 / 28 / 83$

$8 / 11 / 83$

5.4

6.3

0.9

205.

20.5

0.1

90.0

104.24

103.94

0.30

$1.46 \%$

0.2884

0.2862

0.0022
12

$7 / 28 / 83$
$8 / 11 / 83$
5.4
6.7
1.3
456.
15.2
0.3
90.0
98.65
98.31
0.34
$2.24 \%$
0.9500
0.9477
0.0023

20

7/28/83

$8 / 11 / 83$

5.4

6.8

1.4

743.

14.9

5.5

90.0

98.95

98.37

0.58

$3.89 \%$

1.4170

1.4141

0.0029
21

$7 / 28 / 83$

$8 / 11 / 83$

5.4

6.9

1.5

749.

25.0

0.5

90.0

99.86

99.55

0.31

$2.07 \%$

1.5994

1.5963

0.0031

\section{CONSTITUENT ANALYSIS(ng/1fter)}

Analyt Ical Result

SAMPLE ID 4

$\begin{array}{lll}\text { Al } & 2.17 & 3.50 \\ \text { B } & 2.34 & 4.26 \\ \text { Fe } & 0.226 & 0.639 \\ \text { Li } & 2.62 & 5.37 \\ \text { Mn } & 0.077 & 0.222 \\ \text { Mi } & 0.015 & 0.033 \\ \text { Si } & 23.0 & 39.1 \\ \text { Sr } & 0.022 & 0.018 \\ \text { Zr } & 0.005 & 0.017 \\ \text { Ca } & 0.383 & 0.219 \\ \text { K } & 8.260 & 0.302 \\ \text { Mg } & 0.059 & 0.074 \\ \text { MI } & 10.1 & 17.7\end{array}$

20

3.85

4.98

0.885

5.37

0.222

0.039

44.4

0.014

0.035

0.184

0.271

0.081

20.6
Corrected for Blank

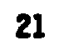

C4

C12

C2O

C21

$\begin{array}{llll}2.16 & 3.49 & 3.84 & 3.96\end{array}$

$\begin{array}{llll}2.34 & 4.26 & 4.98 & 5.05\end{array}$

$\begin{array}{llll}0.214 & 0.627 & 0.873 & 1.05\end{array}$

$\begin{array}{llll}2.62 & 5.37 & 5.37 & 5.55\end{array}$

$\begin{array}{llll}0.077 & 0.222 & 0.222 & 0.246\end{array}$

$\begin{array}{lllll}0.015 & 0.033 & 0.039 & 0.042\end{array}$
22.9

39.1

0.018

44.4

0.014

0.035

0.184

0.271

0.2710 .406

0.383

0.017

0.219

0.302

0.038

i7.7

45.3

0.016

0.041

0.220

0.023

10.1
0.0410 .062

$20.6 \quad 21.2$ 


\author{
TEST I \\ DE IONIZED MATER EXPERIMENT \\ DHPF GLASS (MOMOLITH) \\ RAH DATA TABULATION - LEACHATE ANALYSIS \\ DAY 28 \\ Analytical Results
}

SAPLE ID

5

6

Start Experiment

$7 / 88 / 83 \quad 7 / 28 / 83$

Stop Interval

Intifel $\mathrm{pH}$

Final $\mathrm{pH}$

Diff (pH) ${ }_{\text {Spec SA }}^{2}$ )

Volume (g)

SAV

Temp $\left({ }^{\circ} \mathrm{C}\right)$

$8 / 25 / 83 \quad 8 / 25 / 33$

$5.4 \quad 6.4$

$7.5 \quad 7.5$

2.122 .1

209.203.

$20.9 \quad 20.3$

$0.1 \quad 0.1$

Initial Heightig)

90.0

90.0

Final Height ( 5 )

Helght Loss(g)

zleach Loss

$104.55 \quad 103.94$

$103.91 \quad 103.38$

0.640 .56

Initial Spec Wt(g)

Final Spec Wt(g)

$3.06 \%$

2.763

0.2987

0.2810

0.2956

0.2783

Spec Ht Loss(g)

0.0031

0.0927

CONSTITUENT ANR YSIS(mg/1fter)

Analytical Results

SAPLE ID

$A 1$
$B$
$F e$
$L i$
$M n$
$M i$
$S f$
Sr
Zr
Ca
$k$
Mg
$M$
5

2.72

3.20

0.239

$3.5 ?$

0.091

0.019

30.0

0.019

0.007

0.293

0.268

0.042

13.6
6

2.62

3.07

0.334

3.26

0.105

0.019

28.7

0.024

0.018

0.349

$<.260$

0.065

12.5
Corrected for Blank

C5

C6

2.69

2.59

3.20

3.07

0.230

3.51

0.325

0.091

3.26

0.019

29.8

0.019

0.007

0.105

0.019

28.5

0.293

0.024

0.268

0.018

0.014

13.6
0.349

$<.260$

0.038

18.5 
TEST I

DE IONIZED MATER EXPERINEKT

DUPF GLASS (MOMOLITH)

RAH DATA TABULATION - LEACHATE MNYYSIS

DAY 28

Analyt fcal Results

SAPLE ID

13

14

Start Experiment

Stop Interval

Inftial $\mathrm{pH}$

Final pH

Diff (pH)

Spec SA $\left(m^{2}\right)$

Volume (a)

SNV

Temp $\left({ }^{\circ} \mathrm{C}\right)$

Inftial Neight $(g)$

Final Height $(g)$

Weight Loss(g)

Yleach Loss

Inftial Spec $w t(g)$

Final Spec Wt (g)

Spec Wt Loss(g)

$\begin{array}{ll}7 / 28 / 83 & 7 / 28 / 83 \\ 8 / 25 / 83 & 8 / 25 / 83 \\ 5.4 & 5.4 \\ 8.0 & 8.0 \\ 2.6 & 2.6 \\ 448 . & 450 . \\ 14.9 & 15.0 \\ 0.3 & 0.3 \\ 90.0 & 90.0 \\ 99.01 & 99.51 \\ 98.53 & 98.94 \\ 0.48 & 0.57 \\ 3.22 \% & 3.804 \\ 0.9314 & 0.9254 \\ 0.9293 & 0.9232 \\ 0.0021 & 0.0022\end{array}$

CONSTITLENT AMALYSIS(mg/litter)

Analytical Results

SAPLE ID

$A 1$
$B$
Fe
LI
Mn
N1
S1
Sr
Zr
Ca
$K$
Mg
$M a$
14

3.63

4.85

1.14

5.46

0.267

0.036

43.7

0.022

0.031

0.321

$<.260$

M

20.0
Corrected for Blank

C13 $\quad 14$

$3.30 \quad 3.60$

$4.50 \quad 4.85$

$1.28 \quad 1.13$

$5.18 \quad 5.46$

$0.304 \quad 0.267$

$0.040 \quad 0.036$

40.4. $\quad 43.5$

$0.026 \quad 0.022$

$0.039 \quad 0.031$

$0.294 \quad 0.321$

$<.260 \quad<.260$

$M \quad M$

$19.1 \quad 20.0$

Mote: M - elemental analysis not available. 
TEST I

DEIOHIZED MATER EXPERIMENT

DUPF GLASS (MOMOLITH)

RAH DATA TABULATION - LEACHATE AMLLYSIS

DAY 28

Analyt Ical Result

SAMPLE ID

22

23

Start Experiment

Stop Interval

Inttial pH

Final pH

Diff $\left.\left(\mathrm{pH}^{2}\right) \mathrm{a}^{2}\right)$

Volume $(g)$

SNV

Temp $\left({ }^{\circ} \mathrm{C}\right)$

Initlal Height(g)

Final Weight $(g)$

Weight Loss(g)

zLeach Loss

Initial Spec $N($ (g)

Final Spec Wt $(g)$

Spec it Loss(g)

$7 / 28 / 83$

$8 / 25 / 83$

5.4

8.5

3.1

751.

15.0

0.5

90.0

99.95

99.40

0.55

3.678

1.5187

1.5162

0.0025
7/28/83

$8 / 25 / 83$

5.4

8.5

3.1

736.

14.7

0.5

90.0

99.77

99.23

0.54

3.678

1.4517

1.4484

0.0033

\section{CONSTITUENT ANLLYIS(mg/liter)}

Analyt ical Result

Corrected for Blank

SAMLE ID

22

23

C22

C23

$\begin{array}{lll}\text { Al } & 3.96 & 4.04 \\ \text { B } & 5.59 & 5.59 \\ \text { Fe } & 1.63 & 1.94 \\ \text { L1 } & 6.04 & 5.88 \\ \text { Wn } & 0.366 & 0.423 \\ \text { W1 } & 0.047 & 0.062 \\ \text { S1 } & 47.9 & 48.3 \\ \text { Sr } & 0.021 & 0.023 \\ \text { Zr } & 0.046 & 0.057 \\ \text { Ca } & 0.284 & 0.308 \\ K & .552 & .429 \\ \text { Hg } & 0.190 & 0.159 \\ \text { Ha } & 23.1 & 22.6\end{array}$

3.93

5.59

1.62

6.04

0.366

0.047

47.7

0.021

0.048

0.284

.552

0.163

23.1
4.01

5.59

1.93

5.88

0.423

0.062

48.1

0.023

0.057

0.308

.429

0.132

22.6 
TEST I

DEIOHIZED MATER EXPERIMENT

DUPF GLASS (MONOLITH)

RAY DATA TABULATION - LEACHATE MIALYSIS

DAY 56

Analytical Result

SAPLE IO

7

8

Start Experiment

$\begin{array}{ll}7 / 28 / 83 & 7 / 28 / 83 \\ 9 / 22 / 83 & 9 / 22 / 83 \\ 5.4 & 5.4 \\ 6.8 & 6.8 \\ 1.4 & 1.4 \\ 207 . & 186 . \\ 20.7 & 18.6 \\ 0.1 & 0.1 \\ 90.0 & 90.0 \\ 104.31 & 102.18 \\ 102.31 & 100.74 \\ 2.00 & 1.44 \\ 9.66 \% & 7.74 \% \\ 0.3035 & 0.3731 \\ 0.3000 & 0.3702 \\ 0.0035 & 0.0029\end{array}$

Stop Interval

Initfal $\mathrm{pH}$

Final pH

Diff (pH)

Spec SA $\left(\mathrm{am}^{2}\right)$

Volume (g)

SNY

Temp $\left({ }^{\circ} \mathrm{C}\right)$

Initial Weight(g)

Final Height $(g)$

Weight Loss( $g$ )

Xleach Loss

Initial Spec Wt(g)

Final Spec $W t(g)$

Spec Wt Loss(g)

8

CONSTITUENT ARALYSIS(mg//fter)

Analytical Results

SAMPLE ID

$\begin{array}{lll}A 1 & 2.89 & 2.85 \\ \text { B } & 3.78 & 3.73 \\ \text { Fe } & 0.364 & 0.475 \\ \text { Li } & 4.14 & 4.26 \\ \text { Mn } & 0.142 & 0.158 \\ \text { Mi } & 0.021 & 0.022 \\ \text { Si } & 35.1 & 37.8 \\ \text { Sr } & 0.026 & 0.020 \\ \text { Zr } & 0.035 & 0.038 \\ \text { Ca } & 0.243 & 0.253 \\ \text { K } & <.260 & <.260 \\ \text { Mg } & M A & M \\ \text { Ma } & 15.2 & 15.7\end{array}$

Corrected for Blank

C5 $\quad 66$

$\begin{array}{ll}2.69 & 2.64 \\ 3.78 & 3.73 \\ 0.353 & 0.460 \\ 4.14 & 4.26 \\ 0.142 & 0.158 \\ 0.021 & 0.022 \\ 34.9 & 37.6 \\ 0.026 & 0.020 \\ 0.035 & 0.038 \\ 0.243 & 0.253 \\ 6.260 & 2.260 \\ M & 1 M \\ 15.2 & 15.7\end{array}$

Note: $M A$ - elemental analysis not avaflable. 


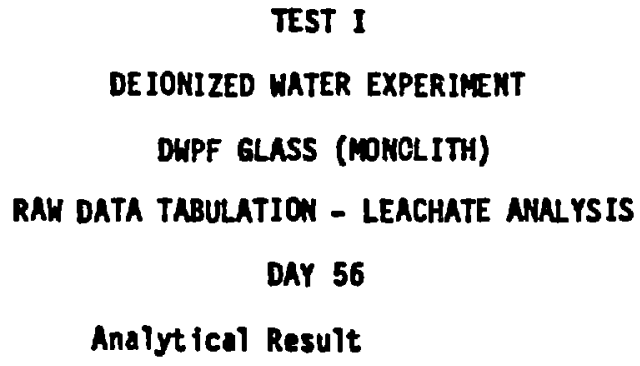

SAPLE ID

15

16

Start Experinent

Stop Interval

Initial pH

Final PH

Diff (pH) $S \mathrm{sm}^{2}$ )

Yol ume (g)

SNV

Temp $\left({ }^{\circ} \mathrm{C}\right)$

Initfal Weight (o)

Final Weight(g)

Weight Loss(g)

ZLeach Loss

Initial Spec Nt(g)

Final Spec Ut $(g)$

Spec Ht Loss(g)

$\begin{array}{ll}7 / 28 / 83 & 7 / 28 / 83 \\ 9 / 22 / 83 & 9 / 22 / 83 \\ 5.1 & 5.4 \\ 7.3 & 7.3 \\ 1.9 & 1.9 \\ 450 . & 450 . \\ 15.0 & 15.0 \\ 0.3 & 0.3 \\ 90.0 & 90.0 \\ 99.17 & 98.73 \\ 98.10 & 97.34 \\ 1.07 & 1.39 \\ 7.13 \% & 9.274 \\ 0.9273 & 0.9305 \\ 0.9244 & 0.9278 \\ 0.0029 & 0.0027\end{array}$

CONSTITUENT AMALYSIS(mg/1iter)

Analytical Results

SAPPLE ID

A1

B

Fe

Li

in

NI

si

Sr

Zr

Ca

ing

Na
15

3.90

5.67

1.83

6.49

0.441

0.048

49.1

0.023

0.034

0.299

$<.260$

M

23.8
16

3.92

5.72

1.85

6.45

0.457

0.047

49.5

0.028

0.035

0.299

$<.260$

$\mathrm{M}$

23.6
Corrected for Blank

$\begin{array}{ll}C 15 & C 16 \\ 3.69 & 3.71 \\ 5.67 & 5.72 \\ 1.82 & 2.84 \\ 6.49 & 6.45 \\ 0.441 & 0.457 \\ 0.048 & 0.047 \\ 49.0 & 49.4 \\ 0.023 & 0.098 \\ 0.034 & 0.035 \\ 0.299 & 0.299 \\ <.260 & <.260 \\ M & M M \\ 23.8 & 23.6\end{array}$

Note: $M$ - elemental analysis not avafiable. 


\section{Appendix B. \\ Test I: DWPF Glass-Deionized Water Experiment, Crushed Samples}




\section{TEST - I \\ DEIONIZED WATER EXPERIMENT}

DHPF GLASS (CRUSHED)

\begin{tabular}{|c|c|c|c|c|c|c|}
\hline NO. & $\mathrm{sAn}^{\left.S A^{2}\right)}$ & $\begin{array}{l}\text { INITYAL } \\
\text { MASSIOL }\end{array}$ & SA/V & $\begin{array}{c}\text { INTERVAL } \\
\text { TIME (d) }\end{array}$ & $\begin{array}{l}\text { TIME } \\
\text { START }\end{array}$ & $\begin{array}{l}\text { TIME } \\
\text { STOP } \\
\end{array}$ \\
\hline $\begin{array}{l}1 \\
2 \\
3 \\
4 \\
5 \\
6\end{array}$ & $\begin{array}{l}20.01 \\
20.01 \\
20.01 \\
20.01 \\
20.01 \\
20.01\end{array}$ & $\begin{array}{l}0.2990 \\
0.2990 \\
0.2990 \\
0.2990 \\
0.2990 \\
0.2990\end{array}$ & $\begin{array}{l}1.0 \\
1.0 \\
1.0 \\
1.0 \\
1.0 \\
1.0\end{array}$ & $\begin{array}{r}1 \\
3 \\
7 \\
14 \\
28 \\
28\end{array}$ & $\begin{array}{l}6 / 4 / 84 \\
6 / 1 / 84 \\
6 / 4 / 84 \\
n \\
n\end{array}$ & $\begin{array}{l}6 / 5 / 84 \\
6 / / 4 / 84 \\
6 / 11 / 84 \\
6 / 18 / 84 \\
7 / 2 / 84\end{array}$ \\
\hline $\begin{array}{r}9 \\
10 \\
11 \\
12 \\
13\end{array}$ & $\begin{array}{l}6.025 \\
6.025 \\
6.360 \\
6.025 \\
6.025\end{array}$ & $\begin{array}{l}0.0900 \\
0.0900 \\
0.0950 \\
0.0900 \\
0.0900\end{array}$ & $\begin{array}{l}.3 \\
.3 \\
.3 \\
.3 \\
.3\end{array}$ & $\begin{array}{l}3 \\
7 \\
14 \\
28.9 \\
61\end{array}$ & $\begin{array}{l}6 / 24 / 84 \\
6 / 20 / 84 \\
6 / 24 / 84 \\
6 / 20 / 84\end{array}$ & $\begin{array}{l}6 / 27 / 84 \\
7 / 8 / 84 \\
7 / 19 / 84 \\
8 / 20 / 84\end{array}$ \\
\hline $\begin{array}{l}14 \\
15 \\
16 \\
17 \\
18\end{array}$ & $\begin{array}{l}10.04 \\
10.04 \\
10.37 \\
10.04 \\
10.04\end{array}$ & $\begin{array}{l}0.1500 \\
0.1500 \\
0.1550 \\
0.1500 \\
0.1500\end{array}$ & $\begin{array}{l}.5 \\
.5 \\
.5 \\
.5 \\
.5\end{array}$ & $\begin{array}{l}3 \\
7 \\
14 \\
28.9 \\
61\end{array}$ & $\begin{array}{l}6 / 24 / 84 \\
6 / 20 / 84 \\
6 / 24 / 84 \\
6 / 20 / 84\end{array}$ & $\begin{array}{l}6 / 27 / 84 \\
7 / 8 / 84 \\
7 / 19 / 84 \\
8 / 20 / 84\end{array}$ \\
\hline
\end{tabular}




$$
\text { TEST - I }
$$

DEIONIZED HATER EXPERIMENT

DHPF GLASS (CRUSHED)

\begin{tabular}{|c|c|c|c|c|c|c|}
\hline No. & $\operatorname{HATER}(g)$ & $\begin{array}{l}\text { INITIAL } \\
\text { NT. }(0)\end{array}$ & $\begin{array}{l}\text { FIMAL } \\
\text { NT. (Q) }\end{array}$ & $\operatorname{LOSS}\left(\frac{\operatorname{LE}}{\mathrm{Q}}\right)$ & $\begin{array}{l}\text { IANT } \\
\text { LOSS(x) }\end{array}$ & 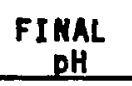 \\
\hline $\begin{array}{l}1 \\
2 \\
3 \\
4 \\
5 \\
6\end{array}$ & $\begin{array}{l}20.0 \\
20.0 \\
20.0 \\
20.0 \\
20.0 \\
20.0\end{array}$ & $\begin{array}{l}99.29 \\
98.08 \\
99.26 \\
98.75 \\
99.83 \\
98.46\end{array}$ & $\begin{array}{l}99.22 \\
97.97 \\
99.11 \\
98.47 \\
99.31 \\
98.07\end{array}$ & $\begin{array}{l}0.07 \\
0.11 \\
0.15 \\
0.28 \\
0.52 \\
0.39\end{array}$ & $\begin{array}{l}0.35 \% \\
0.55 \% \\
0.75 \% \\
1.40 \% \\
2.60 \% \\
1.95 \%\end{array}$ & $\begin{array}{r}8.33 \\
10.05 \\
9.98 \\
9.49 \\
9.51 \\
10.00\end{array}$ \\
\hline $\begin{array}{r}9 \\
10 \\
11 \\
12 \\
13\end{array}$ & $\begin{array}{l}20.0 \\
20.0 \\
20.0 \\
20.0 \\
20.0\end{array}$ & $\begin{array}{l}97.09 \\
97.49 \\
98.20 \\
97.38 \\
98.33\end{array}$ & $\begin{array}{l}96.98 \\
96.72 \\
97.91 \\
96.83 \\
97.30\end{array}$ & $\begin{array}{l}0.11 \\
0.77 \\
0.29 \\
0.55 \\
1.03\end{array}$ & $\begin{array}{l}0.55 \% \\
3.85 \% \\
1.45 \% \\
2.75 \% \\
5.15 \%\end{array}$ & $\begin{array}{l}8.20 \\
9.25 \\
9.52 \\
9.74 \\
9.73\end{array}$ \\
\hline $\begin{array}{l}14 \\
15 \\
16 \\
17 \\
18\end{array}$ & $\begin{array}{l}20.0 \\
20.0 \\
20.0 \\
20.0 \\
20.0\end{array}$ & $\begin{array}{l}97.45 \\
99.32 \\
97.65 \\
97.01 \\
99.33\end{array}$ & $\begin{array}{l}97.33 \\
99.15 \\
97.36 \\
96.49 \\
98.46\end{array}$ & $\begin{array}{l}0.12 \\
0.17 \\
0.29 \\
0.52 \\
0.87\end{array}$ & $\begin{array}{l}0.60 \% \\
0.85 \% \\
1.45 \% \\
2.60 \% \\
4.35 \%\end{array}$ & $\begin{array}{l}9.73 \\
9.76 \\
9.91 \\
9.80 \\
9.84\end{array}$ \\
\hline
\end{tabular}


TEST I

DE IONIZED HATER EXPERIMENT

DHPF GLASS (CRUSHED)

RAH DATA TABULATIOH - LEACHATE AMLLYSIS

OAY 1

Analytical Results

SAPLE IO

Start Experiment

Stop Interval

Initial $\mathrm{pH}$

Final pH

Diff (pH) $S A^{2}\left(\mathrm{~mm}^{2}\right)$

Volume (iil)

SAN

Temp $\left({ }^{\circ} \mathrm{C}\right)$

Initial Weight $(g)$

Final Neight $(g)$.

Weight Loss(g)

Zleach Loss

Initial Spec Wt $(g)$

Final Spec Ht (g)

Spec Wt Loss(g)
1

$6 / 4 / 84$

$6 / 5 / 84$

6.00

8.33

2.33

2000.

20.0

1.0

90.0

99.29

99.22

0.07

$0.35 \%$

0.2990

-

-

\section{CONSTITUENT ANALYSIS(mg/lițer)}

Analytical Result

Corrected for Blank

SAMPLE ID

1

C1

AI

2.75

2.61

2.73

B

0.652

2.59

2.79

0.632

Li

0.175

2.79

Mn

0.039

0.173

23.2

0.038

Si

$\$ 0.002$

23.1

0.014

$<0.002$

0.346

0.012

0.154

0.346

10.7

0.150

10.3 
TEST I

DEIONIZED WATER EXPERIMENT

DLPF GLASS (CRUSHED)

RAH ORTA TABULATION - LEACHATE AWL YSIS

DAY 3

Analytical Results

SAPLE ID

Start Experiment

Stop Interval

Initial pH

Final $\mathrm{pH}$

Diff (pH)
Spec SA $\left(\mathrm{mm}^{2}\right)$

Volume (mi)

SN/V

Temp $\left({ }^{\circ} \mathrm{C}\right)$

Intt fal Height $(g)$

Final Weight $(g)$

Neight Loss $(g)$

ZLeach Loss

Initial Spec $W t(g)$

Final Spec Wt(g)

Spec Wt Loss $(g)$
14

$6 / 24 / 84 \quad 6 / 1 / 84$

\section{$6 / 24 / 84$}

$6 / 27 / 84$

6.0

8.20

2.20

603.

20.0

0.3

90.0

97.09

96.98

0.11

$0.55 \%$

0.0900

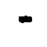

CONSTITUENT AMALYSIS(mg/liter)
$6 / 27 / 84 \quad 6 / 11 / 84$

6.0

9.73

3.73

1004 .

20.0

0.5

90.0

97.45

97.33

0.12

$0.60 \%$

0.1500
6.0

10.05

4.05

2001.

20.0

1.0

90.0

98.08

97.97

0.11

$0.55 \%$

0.2990
Analytical Result

SAMPLE ID

AI
B
Fe
Li
Mn
MI
Si
Sr
Zr
Ca
Mg
Ma
9

2.34

2.31

0.888

2.40

0.275

0.047

21.0

0.015

0.053

0. 389

0.074

9.01
14

2.95

3.10

0.820

3.24

0.227

0.039

27.3

0.012

0.050

0.191

0.051

12.1
Corrected for Blank

c9

C14

C2

2.32

2.93

4.35

2.29

3.08

4.42

4.44

0.868

2.40

0.800

1.35

4.68

0.378

0.273

3.24

4.68

0.058

0.046

0.225

0.376

38.4

20.9

0.038

0.057

$<0.002$

0.015

27.2

38.3

0.083

0.051

0.012

$<.002$

0.293

0.289

0.048

0.081

0.117

0.070

0.191

0.293

17.7
0.047

11.9
0.113

17.5 
TEST I

DEIONIZED WATER EXPERIMENT

DUPF GLASS (CRUSHED)

RAK DATA TABULATIOH - LEACHATE AKALYSIS

DAY 7

Analytical Result

SAPLE ID

Start Experiant

Stop Interval

Initial pH

Final pH

Diff $\left.\left(\mathrm{pH}^{H}\right) \mathrm{mon}^{2}\right)$

Volume (g)

SNY

Temp $\left({ }^{\circ} \mathrm{C}\right)$

Initial Weight(g).

Final Weight $(g)$

Weight Loss(g)

KLeach Loss

Initial Spec Wt(g)

Final Spec Wt $(g)$

Spec Wt Loss(g)
10

\section{$6 / 20 / 84$}

$6 / 27 / 84$

6.00

9.25

3.25

603.

20.0

0.3

90.0

97.49

96.72

0.77

3.85\%

0.0900

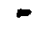

\section{CONSTITUENT ALALYSIS(mg/11ter)}

Analytical Result

$6 / 20 / 84 \quad 6 / 4 / 84$

$6 / 27 / 84 \quad 6 / 11 / 84$

6.00

9.76

3.76

1004.

20.0

0.5

90.0

99.32

99.15

0.17

$0.85 \%$

0.1500

6.00

9.98

3.98

2001.

20.0

1.0

90.0

99.11

99.26

0.15

$0.75 \%$

0.2990

\section{SAPLE ID}

A
$\mathrm{B}$
$\mathrm{Fe}$
$\mathrm{Li}$
$\mathrm{Mn}$
$\mathrm{II}$
$\mathrm{Si}$
$\mathrm{Sr}$
$\mathrm{Zr}$
$\mathrm{Co}$
$\mathrm{Hg}$
10

3.63

3.90

1.15

4.09

0.300

0.045

34.4

0.005

0.092

0.212

0.059

15.2
15

4.21

4.52

2.36

4.77

0.619

0.095

39.7

0.018

0.167

0.288

0.167

17.7
3

\subsection{2}

6.13

2.62

6.49

0.637

0.084

52.0

$<.002$

0.207

0.327

0.186

24.5
Corrected for Blank

C10

C15

C3

\subsection{1}

4.19

4.50

2.34

4.77

0.617

0.094

39.5

0.018

0.165

0.288

0.163

17.5
5.70

6.11

2.60

6.49

0.635

0.083

51.8

$<.002$

0.205

0.327

0.182

24.3 
TEST I

DEIONIZED MATER EXPERIMERT

DUPF CLASS (CRUSHED)

RAH DATA TABULATION - LEACHATE AWLYSIS

DAY 14

Analytical Results

\begin{tabular}{|c|c|c|c|}
\hline & 11 & 6 & 4 \\
\hline 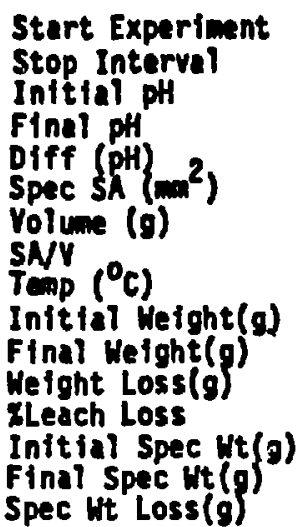 & $\begin{array}{l}6 / 24 / 84 \\
7 / 6 / 843 \\
6.00 \\
9.52 \\
3.52 \\
636 . \\
20.0 \\
0.3 \\
90.0 \\
98.20 \\
97.91 \\
0.29 \\
1.45 \% \\
0.0950\end{array}$ & $\begin{array}{l}6 / 24 / 84 \\
7 / 8 / 84 \\
6.00 \\
9.90 \\
3.90 \\
1037 . \\
20.0 \\
0.5 \\
90.0 \\
97.65 \\
97.36 \\
0.29 \\
1.45 \% \\
0.1550\end{array}$ & 8 \\
\hline
\end{tabular}

CONSTITUENT ANLYYSIS(mg/liter)

Analytical Result

Corrected for Blank

\begin{tabular}{|c|c|c|c|c|c|}
\hline SAPLE ID 11 & 16 & 4 & CII & C16 & C \\
\hline $\begin{array}{l}4.24 \\
4.80 \\
1.69 \\
4.99 \\
0.422 \\
0.049 \\
41.3 \\
0.013 \\
0.129 \\
0.232 \\
0.093 \\
18.6\end{array}$ & $\begin{array}{l}5.28 \\
6.01 \\
3.07 \\
6.29 \\
0.736 \\
0.081 \\
51.1 \\
0.020 \\
0.223 \\
0.348 \\
0.198 \\
23.4\end{array}$ & $\begin{array}{l}6.21 \\
7.23 \\
3.17 \\
7.57 \\
0.715 \\
0.055 \\
59.7 \\
0.008 \\
0.259 \\
0.346 \\
0.206 \\
29.0\end{array}$ & $\begin{array}{l}4.22 \\
4.78 \\
1.67 \\
4.99 \\
0.420 \\
0.048 \\
41.1 \\
0.013 \\
0.127 \\
0.232 \\
0.089 \\
18.4\end{array}$ & $\begin{array}{l}5.26 \\
5.99 \\
3.05 \\
6.29 \\
0.734 \\
0.080 \\
50.9 \\
0.020 \\
0.221 \\
0.348 \\
0.194 \\
23.2\end{array}$ & $\begin{array}{l}6.19 \\
7.21 \\
3.15 \\
7.57 \\
0.713 \\
0.054 \\
59.5 \\
0.008 \\
0.257 \\
0.346 \\
0.202 \\
28.8\end{array}$ \\
\hline
\end{tabular}




\section{TEST I}

DEIOMIZED WATER EXPERINERT

DUPF GLASS (CRUSHED)

RAH DATA TABULATION - LEACHATE AMALYSIS

DAY 28

Analytical Results

\begin{tabular}{|c|c|c|c|c|}
\hline & 12 & 17 & 5 & 6 \\
\hline 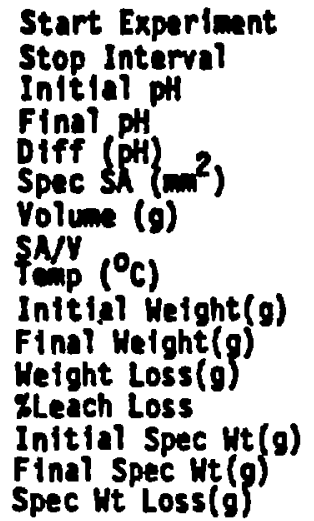 & $\begin{array}{l}6 / 20 / 84 \\
7 / 19 / 84 \\
6.00 \\
9.74 \\
3.74 \\
603 . \\
20.0 \\
0.3 \\
90.0 \\
97.38 \\
96.83 \\
0.55 \\
2.75 \% \\
0.0900\end{array}$ & $\begin{array}{l}6 / 20 / 84 \\
7 / 18 / 84 \\
6.00 \\
9.80 \\
3.80 \\
1004 \\
10.0 \\
9.5 \\
90.0 \\
97.01 \\
96.49 \\
0.52 \\
2.60 \% \\
0.1500\end{array}$ & $\begin{array}{l}6 / 4 / 84 \\
7 / 2 / 84 \\
6.00 \\
9.51 \\
3.51 \\
2001 . \\
20.0 \\
1.0 \\
90.0 \\
99.83 \\
99.31 \\
0.52 \\
2.501\end{array}$ & $\begin{array}{l}6 / 4 / 84 \\
7 / 2 / 84 \\
6.00 \\
10.00 \\
4.00 \\
2001 . \\
20.0 \\
1.0 \\
90.0 \\
98.46 \\
98.07 \\
0.39 \\
1.95 \% \\
0.2990\end{array}$ \\
\hline
\end{tabular}

CONSTITUENT AWLYSIS(ng/11ter)

Analytical Result

Corrected for Blank

\begin{tabular}{|c|c|c|c|c|c|c|c|c|}
\hline NPLE ID & 12 & 17 & 5 & 6 & C12 & a17 & C5 & $C 6$ \\
\hline $\begin{array}{l}\mathrm{Al} \\
8 \\
\mathrm{Fe} \\
\mathrm{LI} \\
\mathrm{Mn} \\
\mathrm{Mi} \\
\mathrm{Si} \\
\mathrm{Sr} \\
\mathrm{Zr} \\
\mathrm{Ca} \\
\mathrm{Hi}\end{array}$ & $\begin{array}{l}5.39 \\
6.10 \\
3.76 \\
6.49 \\
0.918 \\
0.078 \\
52.6 \\
0.033 \\
M \\
0.490 \\
0.265 \\
24.7\end{array}$ & $\begin{array}{l}5.73 \\
6.74 \\
3.58 \\
7.23 \\
0.842 \\
0.058 \\
57.1 \\
0.033 \\
M \\
0.466 \\
0.234 \\
27.5\end{array}$ & $\begin{array}{l}7.08 \\
8.99 \\
5.33 \\
9.34 \\
1.21 \\
0.098 \\
73.8 \\
0.042 \\
0.326 \\
0.504 \\
0.313 \\
34.9\end{array}$ & $\begin{array}{l}6.97 \\
8.73 \\
4.83 \\
9.20 \\
1.13 \\
0.106 \\
71.0 \\
0.029 \\
0.325 \\
0.463 \\
0.273 \\
34.1\end{array}$ & $\begin{array}{l}5.37 \\
6.08 \\
3.74 \\
6.49 \\
0.916 \\
0.077 \\
52.4 \\
0.033 \\
M \\
0.490 \\
0.261 \\
24.5\end{array}$ & $\begin{array}{l}5.71 \\
6.72 \\
3.56 \\
7.23 \\
0.840 \\
0.067 \\
56.9 \\
0.033 \\
11 \\
0.466 \\
0.230 \\
27.3\end{array}$ & $\begin{array}{l}7.06 \\
8.97 \\
5.31 \\
9.34 \\
1.21 \\
0.097 \\
73.6 \\
0.042 \\
0.324 \\
0.504 \\
0.309 \\
34.7\end{array}$ & $\begin{array}{l}6.95 \\
8.71 \\
4.81 \\
9.20 \\
1.13 \\
0.105 \\
70.8 \\
0.029 \\
0.323 \\
0.463 \\
0.269 \\
33.9\end{array}$ \\
\hline
\end{tabular}

Note: $M$ - elemental analys is not avallable. 
TEST I

DEIONIZED WATER EXPERIMENT

DWPF GLASS (CRUSHED)

RAN DATA TABULATIOH - LEACHATE AMLYSIS

DAY 61

Analytical Results

SNPLE ID

13

18

Start Experiment

$6 / 20 / 84 \quad 6 / 20 / 84$

Stop Interval

Inttlal pH

$8 / 20 / 84 \quad 8 / 20 / 84$

Final pH

Diff (pH) ${ }_{\text {Spec } S A}^{2}$ )

6.00

9.73

6.00

3.73

603.

20.0

0.3

SAV

Temp $\left({ }^{\circ} \mathrm{C}\right)$

90.0

Initial Weight(g)

98.33

9.84

3.84

1004.

Final Weight $(g)$

Helght Loss(g)

Eleach Loss

97.30

1.03

20.0

Initia) Spec Wt (g)

Final Spec Wt (g)

$5.15 \%$

0.5

90.0

Spec Wt Loss(g)

0.0900

99.33

98.46

0.87

$4.35 \%$

0.1500
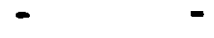

\section{CONSTITUENT ANALYSIS(mg// iter)}

Analyt lcal Result

SAMPLE ID

$A 1$
$\mathrm{~B}$
$\mathrm{Fe}$
$\mathrm{Li}$
$\mathrm{Mn}$
$\mathrm{Mi}$
$\mathrm{Si}$
$\mathrm{Sr}$
$\mathrm{Zr}$
$\mathrm{Ca}$
$\mathrm{Mg}$
$\mathrm{Ma}$
13

5.38

7.02

3.82

7.13

0.985

0.079

59.4

0.050

0.250

0.523

0.254

26.8
18

6.06

8.09

3.79

8.27

0.828

0.064

66.4

0.044

0.280

0.488

0.209

30.9
Corrected for Blank

C13

C18

5.36

6.04

7.00

3.82

7.13

0.983

0.078

59.2

0.050

0.248

0.523

0.250

26.6
8.07

3.79

8.27

0.826

0.063

66.2

0.044

0.278

0.488

0.205

30.7 


\section{Appendix C. \\ Test II: DWPF Glass-J-13 Water Experimen:, Monolith Samples}




\section{TEST II}

J-13 MATER EXPERIMENT

DIPF GLASS (MOMOLITH)

\begin{tabular}{|c|c|c|c|c|c|c|c|c|c|}
\hline \multicolumn{2}{|c|}{ MC. GLASS } & \multirow[b]{2}{*}{411.} & $\begin{array}{l}\text { InITIAL } \\
\text { mSSS(a) }\end{array}$ & \multirow{2}{*}{$\frac{\operatorname{FIMN}_{\text {mSS }}(0)}{2.1377}$} & \multirow{2}{*}{$\frac{\begin{array}{l}\text { MSs } \\
\operatorname{Loss}(a)\end{array}}{0.0001}$} & \multicolumn{2}{|c|}{$\begin{array}{l}\text { SNV INTERVA } \\
\text { TIME(d) }\end{array}$} & \multirow{2}{*}{$\begin{array}{l}\text { TINE } \\
\text { START } \\
2 / 28 / 84\end{array}$} & \multirow{2}{*}{$\frac{\begin{array}{c}\text { TIME } \\
\text { STOP }\end{array}}{3 / 2 / 8}$} \\
\hline 1 & 3 & & 2.1378 & & & .3 & 3 & & \\
\hline 2 & 4 & 403. & 1.3516 & 1.3512 & 0.0004 & .3 & 7 & - & $3 / 6$ \\
\hline 3 & 6 & 402. & 1.0858 & 1.0862 & $0.0004 t$ & .3 & 14 & $n$ & $3 / 13$ \\
\hline 4 & 8 & 404. & 1.2905 & 1.2904 & 0.0001 & .3 & 28 & " & $3 / 29$ \\
\hline 5 & 13 & 399. & 1.3628 & .1 .3629 & $0.0001+$ & .3 & 28 & $"$ & $3 / 29$ \\
\hline 6 & $28 / 19$ & 402. & 0.9273 & 0.9275 & $0.0002+$ & .3 & 56 & “ & $4 / 26$ \\
\hline
\end{tabular}

$\begin{array}{rlllllllll}7 & 2 & 612 . & 2.4224 & 2.4224 & 0.0000 & .5 & 3 & \cdots & 3 / 2 \\ 8 & 11 & 605 . & 2.2957 & 2.2953 & 0.0004 & .5 & 7 & \cdots & 3 / 6 \\ 9 & 12 & 611 . & 2.5058 & 2.5058 & 0.0000 & .5 & 14 & \cdots & 3 / 19 \\ 10 & 9 & 605 . & 2.6077 & 2.6080 & 0.0003+ & .5 & 28 & \cdots & 3 / 29 \\ 11 & 7 \pi / 15 & 584 . & 2.1626 & 2.1628 & 0.0002+ & .5 & 28 & \cdots & 3 / 29 \\ 12 & 17 / 20 & 574 . & 1.5234 & 1.5236 & 0.0002+ & .5 & 56 & \cdots & 4 / 26\end{array}$

13 blank

14 blank

15 blank

16 blank

17 blank

18 blank

$\begin{array}{lll}3 & \cdots & 3 / 2 \\ 7 & \cdots & 3 / 6 \\ 14 & \cdots & 3 / 13 \\ 28 & \cdots & 3 / 29 \\ 28 & \cdots & 3 / 29 \\ 56 & 2 / 28 / 84 & 4 / 26\end{array}$




\section{TEST II}

J-13 MATER EXPERIMENT

DWPF GLASS (MOHOLITH)

\begin{tabular}{|c|c|c|c|c|c|c|}
\hline \multirow[b]{2}{*}{ no. } & \multirow[b]{2}{*}{$J-13(q)$} & \multirow{2}{*}{$\begin{array}{l}\text { INITIAL } \\
\text { W. (g) }\end{array}$} & \multirow{2}{*}{$\begin{array}{l}\text { FIML } \\
\text { NT. (q) }\end{array}$} & \multicolumn{2}{|c|}{ LEACHNT } & \multirow{2}{*}{$\begin{array}{c}\text { FIMNL } \\
\text { pH }\end{array}$} \\
\hline & & & & $\operatorname{Los} 5(0)$ & $\operatorname{LosS}(8)$ & \\
\hline 1 & 13.70 & 98.35 & 98.25 & 0.10 & 0.738 & 9.17 \\
\hline 2 & 13.43 & 96.30 & 95.93 & 0.37 & 2.768 & 9.21 \\
\hline 3 & 13.39 & 97.21 & 96.96 & 0.25 & 2.878 & 9.28 \\
\hline 4 & 13.46 & 97.41 & 96.91 & 0.50 & 3.718 & 9.48 \\
\hline 5 & 13.46 & 97.90 & 97.33 & 0.57 & $4.23 \%$ & 9.41 \\
\hline 6 & 13.42 & 97.36 & 96.37 & 0.99 & $7.38 \%$ & 9.43 \\
\hline 7 & 12.25 & 97.21 & 97.07 & 0.14 & 1.148 & 9.21 \\
\hline 8 & 12.09 & 96.97 & 96.62 & 0.35 & $2.89 \%$ & 9.32 \\
\hline 9 & 12.22 & 95.79 & 96.47 & 0.17 & $2.62 x$ & 9.44 \\
\hline 10 & 12.11 & 97.60 & 96.83 & 0.77 & $6.36 \%$ & 9.64 \\
\hline 11 & 11.67 & 95.98 & 95.46 & 0.52 & 4.538 & 9.35 \\
\hline 12 & 11.48 & 95.51 & 94.40 & 1.11 & 9.678 & 9.51 \\
\hline 13 & 10.00 & 88.66 & 88.53 & 0.13 & $1.30 \%$ & 9.17 \\
\hline 14 & 10.00 & 88.83 & 88.61 & 0.22 & $2.20 \%$ & 9.31 \\
\hline 15 & 10.00 & 88.70 & 88.25 & 0.45 & $4.50 \%$ & 9.60 \\
\hline 16 & 10.00 & 88.62 & 87.89 & 0.73 & $7.30 \%$ & 9.50 \\
\hline 17 & 10.00 & 88.77 & 88.21 & 0.56 & $5.60 x$ & 9.41 \\
\hline 18 & 10.00 & 88.82 & 87.51 & 1.31 & $13.10 \%$ & 9.54 \\
\hline
\end{tabular}




\section{TEST II}

J-13 WATER EXPERIMENT

DUPF GLASS (MONOLITH)

DAY 3 - ICP Analysis

SAMPLE

$S N / V=0.3$

ELEM DET. LIM.

$\begin{array}{ll}\mathrm{Al} & .012 \\ \mathrm{~B} & .024 \\ \mathrm{Fe} & .003 \\ \mathrm{Li} & .002 \\ \mathrm{Hn} & .0003 \\ \mathrm{Hi} & .008 \\ \mathrm{Si} & .020 \\ \mathrm{Sr} & .016 \\ \mathrm{Zr} & .002 \\ \mathrm{Ca} & .004 \\ \mathrm{~K} & .820 \\ \mathrm{Hg} & .001 \\ \mathrm{Ka} & .028\end{array}$

0.5

7

.874

$.258(.10)$

.475

$.581(.57)$

.034

.011

40.5

0.053

NA

12.3

16.3

.207

47.7

BLANK

13

.804

$.193(.19)$

.028

$.048(.40)$

.003

.007

40.2

0.049

MA

12.5

17.0

.148

47.9

.157

47.6

Note: - Values in parentheses were analyzed on a different system. MA - elemental analysis not available. 


\section{TEST II}

J-13 HATER EXPERIMENT

DHPF GLASS (MONOLITH).

DAY 7- ICP Analysis

SAMPLES

SA/Y=0.3 $\quad 0.5 \quad$ BLANK

ELEM DET. LIM.

$\begin{array}{lllll}\text { Al } & .012 & .832 & .986 & .795 \\ \text { B } & .024 & .232(.24) & .223(.22) & .195(.20) \\ \text { Fe } & .003 & .283 & .068 & .028 \\ \text { Li } & .002 & .278(.28) & .438(.44) & .049(.07) \\ \text { Mn } & .0003 & .042 & .016 & .002 \\ \text { Wi } & .008 & .058 & .009 & 0.002 \\ \text { Si } & .020 & 40.1 & 40.9 & 39.9 \\ \text { Sr } & .016 & .051 & .049 & 0.045 \\ \text { Zr } & .002 & \text { HA } & \text { NA } & \text { HA } \\ \text { Ca } & .004 & 12.4 & 12.4 & 12.8 \\ \text { K } & .820 & 13.7 & 13.6 & 13.4 \\ \text { Hg } & .001 & 0.175 & 0.154 & 0.152 \\ \text { Wa } & .028 & 48.2 & 48.5 & 48.8\end{array}$

Note: Values in parentheses were analyzed on a different systen. MA - elemental analysis not avallable. 
TEST II

J-13 WATER EXPERIMENT

DUPF GLASS (MONOLITH)

DAY 24- Iep Analysis

SAMPLES

$\underline{S A} Y Y=0.3 \quad 0.5 \quad$ BLANK

ELEM DET. LIM. 3

9

15

$\begin{array}{lllll}\mathrm{Al} & .012 & .989 & .809 & .832 \\ \mathrm{~B} & .024 & .240 & .232 & .195 \\ \mathrm{Fe} & .003 & .201 & .135 & .066 \\ \mathrm{LI} & .002 & .358 & .406 & .046 \\ \mathrm{Hn} & .0003 & .030 & .023 & .004 \\ \mathrm{KI} & .008 & .023 & .018 & .011 \\ \mathrm{SI} & .020 & 35.0 & 36.2 & 36.5 \\ \mathrm{Sr} & .016 & .109 & .108 & .114 \\ \mathrm{Zr} & .002 & \mathrm{NA} & \mathrm{NA} & \mathrm{KA} \\ \mathrm{Ca} & .004 & 10.5 & 10.5 & 10.9 \\ \mathrm{~K} & .820 & 13.2 & 12.8 & 13.1 \\ \mathrm{Hg} & .001 & .137 & .123 & .131 \\ \mathrm{Ha} & .028 & 43.2 & 42.9 & 42.6\end{array}$

Note: MA - elemental analysis not avallable. 
TEST II

J-13 MATER EXPERIMENT

DUPF GLASS (MONOLITH)

DAY 28- ICp Analysis

\begin{tabular}{|c|c|c|c|c|c|c|}
\hline \multirow[b]{2}{*}{$\frac{\text { ELEM }}{\lambda 1}$} & \multicolumn{2}{|r|}{ SAMPLE } & \multicolumn{2}{|c|}{$S A M=0.5$} & \multicolumn{2}{|c|}{ BLANKS } \\
\hline & $\frac{4}{.492}$ & $\frac{5}{.518}$ & $\begin{array}{c}10 \\
.467\end{array}$ & $\frac{11}{.709}$ & $\begin{array}{c}16 \\
.550\end{array}$ & $\frac{17}{.699}$ \\
\hline - & $.304(.30)$ & $.261(.26)$ & $.546(.49)$ & $.235(.22)$ & $.208(.20)$ & $.212(.1$ \\
\hline Fe & .027 & .039 & 4.92 & .022 & .029 & .505 \\
\hline LI & $.521(.52)$ & $.499(.50)$ & .989 & .361 & $.052(.06)$ & .0521 .0 \\
\hline$M n$ & .002 & $<.002$ & .004 & $<.002$ & $<.002$ & .010 \\
\hline Mi & $<.008$ & $<.008$ & $<.008$ & $<.008$ & P.008 & $<.008$ \\
\hline si & 36.7 & 38.6 & 37.2 & 37.2 & 38.6 & 38.5 \\
\hline sr & .057 & .060 & .050 & .061 & .057 & .054 \\
\hline $2 r$ & $M A$ & MA & $M A$ & MA & MA & HA \\
\hline Ca & 10.7 & 8.01 & 7.41 & 5.95 & 5.65 & 7.06 \\
\hline $\mathbf{K}$ & 24.2 & 18.3 & 14.0 & 16.7 & 13.0 & 12.6 \\
\hline Mg & .038 & .049 & .029 & .030 & .028 & .161 \\
\hline Me & 51.5 & 53.3 & 54.8 & 51.5 & 51.5 & 52.0 \\
\hline
\end{tabular}

Note: The values in parentheses were analyzed on a different syster MA - elemental inalysis not avallable. 


\title{
TEST II
}

\author{
J-13 MATER EXPERIMENT \\ DKPF GLASS (MONOLITH)
}

DAY 56- Iep Analysis

SAMPLES

$S A / Y=0.3$

ELEM DET LIM

$\begin{array}{ll}\mathrm{Al} & .012 \\ \mathrm{~B} & .024 \\ \mathrm{Fe} & .003 \\ \mathrm{LI} & .002 \\ \mathrm{Hn} & .0003 \\ \mathrm{HI} & .008 \\ \mathrm{SI} & .020 \\ \mathrm{Sr} & .026 \\ \mathrm{Ir} & .002 \\ \mathrm{Ca} & .004 \\ \mathrm{~K} & .820 \\ \mathrm{Hg} & .001 \\ \mathrm{Ha} & .028\end{array}$

0.5

12

2.09

.517

.317

1.36

.098

.019

45.8

.028

.008

12.3

16.1

.113

60.4
BLANK

18

1.15

.230

.857

.053

.005

.003

45.6

.025

C.002

14.8

18.7

.179

59.1 
TEST II

J-12 WATER EXPERIMENT

DWPF GLASS (MONOLITH)

RAH DATA TABULATIOH - LEACHATE AULYSIS

DAY 3

Analytical Result

SAMPLE ID

Start Experiment

1

7

Stop Interval

$2 / 28 / 84$

$2 / 28 / 84$

Initial pH

$3 / 2 / 84$

$3 / 2 / 84$

Final pH

Diff (pH) ${ }^{2}$ )

8.78

8.78

9.17

0.39

411.

Volume(g)

SNV

13.70

0.3

90.0

9.21

0.43

Temp $\left({ }^{\circ} \mathrm{C}\right)$

98.35

612.

Inttial Weight/gr

Final Weight (g)

Weight Loss(g)

ZLeach Loss

98.25

0.10

12.25

0.5

0.738

Initial Spec Nt(g)

Final Spec wt $(g)$

Spec Wt Loss(g)

2.1378

2.1377

90.0

0.0001

97.21

97.07

0.14

$1.14 \%$

2.4224

2.4224

0.0000

CONSTITUENT AMALYSIS(mg/liter)

Analytical Result

Corrected for Blank

SAMPLE IO

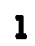

7

Cl

C7

A1

0.888

0.196

0.874

0.258

0.084

0.003

0.070

0.255

0.475

0.227

0.065

0.048

0.581

0.000

0.447

0.019

0.034

0.016

0.533

0.043

0.011

10.3

40.5

0.052

0.053

M

12.5

26.2

MA

Ca

K

0.148

12.3

16.3

47.9

0.207

47.7

0.036

0.1

0.031

0.004

0.003

0.3

M

0.004

0.0

$M$

9.2

$-.009$

0.3

$-.2$

$-0.7$

0.050

0.1

Note: MA - elemental analysis not avallable. 
TEST II

J-13 WATER EXPERIMENT

DWPF GLASS (MONOLITH)

RAW DATA TABULATION - LEACHATE AMALYSIS

DAY 7

Analytfeal Result

SAPLE ID

2

8

Start Experiment

$2 / 28 / 84$

$2 / 28 / 84$

Stop Interval

Inftial pH

final $\mathrm{PH}$

Diff (pH)

Spec SA $\left(\mathrm{m}^{2}\right)$

Yolume (g)

SNV

$\left({ }^{\circ} \mathrm{C}\right)$

Initfal Welght(g)

Final Weight(g)

Height Loss(g)

MLeach Loss

$3 / 6 / 84$

8.78

9.21

403.

13.43

0.3

90.0

96.30

95.93

0.37

$3 / 6 / 84$

Initial Spec Ut $(g)$

Final Spec Ut(g)

Spec Wt Loss(g)

$2.76 \%$

1.3516

1.3512

0.0004

8.78

9.32

605.

12.09

0.5

90.0

96.97

96.62

0.35

2.897

2.2957

2.2953

0.0004

CONSTITUENT ARALYSIS(mg/liter)

Analytical Result

Corrected for Blank

SAMPLE ID

2

8

C2

C8

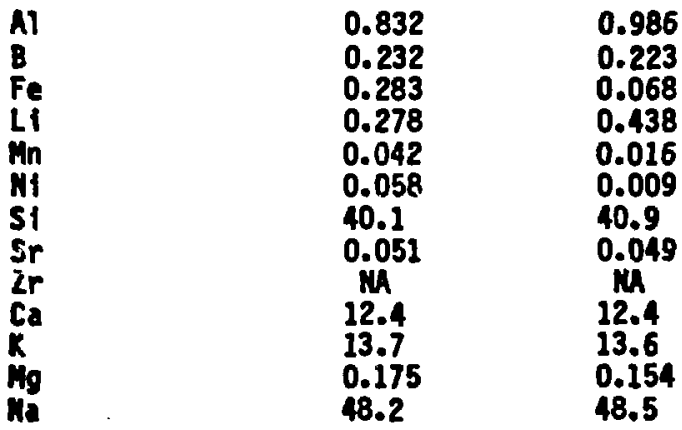

0.037

0.191

0.037

0.028

0.255

0.040

0.229

0.389

0.040

0.014

0.042

0.056

0.2

0.006

NA

$-0.4$

0.3

0.023

0.007

48.2

$-0.6$

1.0

0.004

MA

$-0.4$

0.2

0.002

$-0.3$

Hote: $M A$ - elemental analysis not avaflable. 
TEST II

J-13 WATER EXPERIMENT

DUPF GLASS (MONOLITH)

RAN DATA TABULATION - LEACHATE NHALSIS

DAY 14

Analytical Result

SAPLE IO

Start Experiment

Stop Interval

Inttial pH

Final $\mathrm{pH}$

Diff $\left.(\mathrm{pH}) \mathrm{m}^{2}\right)$

volume (g)

SNenp ( $\left.{ }^{\circ} \mathrm{C}\right)$

Inttial Welght (g)

Final Weight $(g)$

Welght Loss(g)

BLeach Loss

Initial Spec Wt(g)

Final Spec Wt(g)

Spec Wt Loss(g)
3

$\begin{array}{ll}2 / 28 / 84 & 2 / 28 / 84 \\ 3 / 13 / 84 & 3 / 13 / 84 \\ 8.78 & 8.78 \\ 9.18 & 9.44 \\ 0.40 & 0.66 \\ 402 . & 611 . \\ 13.39 & 12.22 \\ 0.3 & 0.5 \\ 90.0 & 90.0 \\ 97.21 & 96.79 \\ 96.96 & 96.47 \\ 0.25 & 0.17 \\ 1.87 & 2.62 \\ 1.0858 & 2.5058 \\ 1.0862 & 2.5058 \\ +0.0004 & 0.0000\end{array}$

CONSTITUENT ANR YSIS(ng/Itter)

Analytical Result

$$
3
$$

0.989

0.240

0.201

0.358

0.030

0.023

35.0

D.109

$M$

10.5

13.2

0.137

43.2
9

0.809

0.232

0.135

0.406

0.023

0.018

36.2

0.108

M.

10.5

12.8

0.123

42.9
Corrected for Blank

C3

C9

$\begin{array}{ll}0.157 & -0.023 \\ 0.045 & 0.037 \\ 0.135 & 0.069 \\ 0.312 & 0.360 \\ 0.026 & 0.019 \\ 0.012 & 0.007 \\ -1.5 & -. .3 \\ -.005 & -.006 \\ M & -.4 \\ -.04 & -.4 \\ 0.1 & -0.33 \\ 0.006 & -.008 \\ 0.6 & 0.3\end{array}$

Note: M - elemental analysis not avaflable. 
TEST II

\section{J-13 WATER EXPERIMENT}

DUPF GLASS (MONOLITH)

RAW DATA TABULATION - LEACHATE ANALYSIS

DAY 28 (PAGE 1)

Analytical Result

SNMPLE ID

4

5

Start Experiment

Stop Interval

$2 / 28 / 84$

$2 / 28 / 84$

$3 / 29 / 84 \quad 3 / 29 / 84$

Initial pH

8.78

8.78

Final pH

9.48

9.41

Diff $\left.(\mathrm{pH}) \mathrm{m}^{2}\right)$

0.70

404.

13.46

Volume (g)

SAV

0.3

90.0

Initial We Ight (g)

Fina! Weight(g)

Weight Loss(g)

WLeach Loss

97.41

96.91

0.50

3.71

Initial Spec Wt $(g)$

Final Spec Wt (g)

Spec Wt Loss(g)

1.2905

1.2904

0.0001

0.63

399.

13.46

0.3

90.0

97.90

97.33

0.57

4.23

1.3628

1.3629

$+0.0001$

CONSTITUENT ANALYSIS(mg/liter)

Analytical Result

SAMPLE ID

A]

B

Fe

Li

Nn

Ni

Si

Sr

Zr

Ca

$k$

$\mathrm{Mg}$

$\mathrm{Na}$
4

0.492

0.304

0.027

0.521

0.002

$<.008$

36.7

0.057

NA

10.7

24.2

0.038

$\mathbf{5 1 . 5}$
5

0.518
0.261
0.039
0.499
$<0.002$
$<.008$
38.6
0.060
NA

8.01

18.3

0.049

53.3
Corrected for Blank

$\mathrm{CO4}$

CO5

$\begin{array}{ll}-0.133 & -0.107 \\ 0.094 & 0.051 \\ -0.240 & -0.228 \\ 0.469 & 0.447 \\ 0.002 & <.002 \\ 5.008 & <.008 \\ -1.85 & 0.05 \\ 0.001 & 0.004 \\ \text { NA } & \text { NA } \\ 4.35 & 1.66 \\ 11.4 & 5.5 \\ -0.056 & 0.046 \\ -0.25 & 1.55\end{array}$

Note: NA - elemental analysis not available. 
TEST II

J-13 MATER EXPERIMENT

DHPF GLASS (MONOLITH)

RAH DATA TABULATIOH - LEACHATE AML YSIS

DAY 28 (PAEE 2)

Analytical Result

SAPLE ID

Start Experiment

Stop Interval

Initfal pH

Final $\mathrm{pH}$

Diff (pH) ${ }^{2}$ )

Volume (g)

SNY

Temp ( $\left.{ }^{\circ} \mathrm{C}\right)$

Initial Weight(g)

Finat Height $(g)$

Height Loss(g)

\#leach Loss

Initial Spec Wt(g)

Final Spec Wt(g)

Spec Wt Loss
10

21

2/28/84

$3 / 29 / 84$

8.78

9.64

0.86

605.

12.11

0.5

90.C

97.60

96.83

0.77

6.36

2.6077

2.6080

$+0.0003$
2/28/84

$3 / 29 / 84$

8.78

9.35

0.57

584.

11.67

0.5

90.0

95.98

95.46

0.52

4.53

2.1626

2.1628

$+0.0002$

CONSTITUENT AKALYSIS(mg/liter)

Analytical Resuit

SAMPLE ID

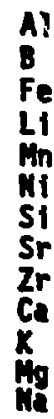

10

0.467

0.546

4.92

0.989

0.004

$\$ 0.008$

37.2

0.050

M

7.41

14.0

0.029

54.8
11

0.709

0.235

0.022

0.361

$<0.002$

$<0.008$

37.2

0.061

$M$

5.95

16.7

0.030

51.5
Corrected for Blank

C10 C11

$\begin{array}{ll}-0.158 & 0.085 \\ 0.336 & 0.025 \\ 4.65 & -0.25 \\ 0.937 & 0.309 \\ 0.004 & 2.002 \\ 8.008 & 5.008 \\ -1.35 & -1.35 \\ 0.001 & 0.004 \\ 1 M & M \\ 1.06 & -0.41 \\ 1.2 & 3.9 \\ -0.066 & -0.065 \\ 3.05 & -0.25\end{array}$

Note: $M$ - elemental analysis not avallable. 
TEST 1 I

J-13 MATER EXPERIMEMT

DUPF GLASS (MNOLITH)

RAW DATA TABULATIOH - LEACHATE AWLYSIS

Dil 56

Analytical Result

SAPLE ID

$6 \quad 12$

Start Experiment

Initial pH

Final pH

Diff (pH)

Stop Intervg!

Spec SA ( $\left.\mathrm{mm}^{2}\right)$

Yolume (g)

SNY

Temp $\left({ }^{\circ} \mathrm{C}\right)$

Initial Welght(g)

Final Height $(g)$

Meight Loss(g)

ILeach Loss

Initial Spec Wt(g)

Final Spec Wt (g)

Spec Ut Loss (g)

$2 / 28 / 84 \quad 2 / 28 / 84$

8.78 .8 .78

9.439 .51

$0.65 \quad 0.73$

$4 / 26 / 84 \quad 4 / 26 / 84$

402.5574.

$13.41 \quad 11.48$

$0.3 \quad 0.5$

$90.0 \quad 90.0$

$97.36 \quad 95.51$

$96.37 \quad 94.40$

$0.99 \quad 1.11$

$7.38 \quad 9.67$

$0.9273 \quad 1.5234$

$0.9275 \quad 1.5236$

$+0.0002 \quad+0.0002$

CONSTITUENT ANLAYSIS(mg/11ter)

Analyt ical Result Corrected for Blank

SAPLE IO

6

12

$-.-\quad 66 \quad C 12$

Al

1.14

0.499

1.09

0.573

0.517

Fe

0.965

0.317

0.121

1.36

in

0.027

0.098

$45 . \mathrm{C}$

D.019

S1

0.056

45.8

Sr

0.009

0.028

10.4

0.008

ca

25.9

0.130

12.3

16.1

$\mathrm{Mg}$

58.1

0.113

60.4

$\begin{array}{ll}-.01 & -.06 \\ 0.259 & 0.287 \\ -.284 & -.540 \\ 0.912 & 1.31 \\ 0.116 & 0.093 \\ 0.024 & 0.016 \\ -.6 & -.2 \\ 0.001 & 0.003 \\ 0.009 & 0.008 \\ -4.4 & -2.5 \\ 7.2 & -2.6 \\ -.049 & -.066 \\ -1.0 & 1.3\end{array}$




\section{Appendix : . \\ Test III: DWPF Glass-J-13 Water + Tuff Experiment, Monolith Samples}


TEST III

\section{\-13 WATER EXPERIMENT WITH TUFF}

DUPF GLASS (MOMOLITH)

\begin{tabular}{|c|c|c|c|c|c|c|c|c|}
\hline ). & $\begin{array}{c}\text { GLASS } \\
\text { (1) }\end{array}$ & $\begin{array}{l}\text { S.R. } \\
\text { (mm2) }\end{array}$ & $\begin{array}{r}\text { INITLAL } \\
\text { NT(g) }\end{array}$ & $\begin{array}{l}\text { FINAL } \\
\text { NT(g) }\end{array}$ & $\begin{array}{l}\text { MSS } \\
105 S(g)\end{array}$ & SNN INTE & $\begin{array}{l}\text { TIME } \\
\text { START }\end{array}$ & $\begin{array}{l}\text { TIME } \\
\text { STOP } \\
\end{array}$ \\
\hline $\begin{array}{l}5 \\
6 \\
1 \\
7\end{array}$ & $\begin{array}{c}8 \\
5 \\
134 \\
4 \\
2\end{array}$ & $\begin{array}{l}298 \\
252 \\
212 \\
305 \\
355\end{array}$ & $\begin{array}{l}0.4094 \\
0.6230 \\
0.3920 \\
0.8025 \\
0.9009\end{array}$ & $\begin{array}{l}0.4095 \\
0.6230 \\
0.3919 \\
0.8024 \\
0.9001\end{array}$ & $\begin{array}{l}+.0001 \\
0.0000 \\
0.0001 \\
0.0001 \\
0.0008\end{array}$ & {$\left[\begin{array}{cc}0.1 & 7 . \\
0.1 & 14 . \\
0.1 & 28 . \\
0.1 & 28 . \\
0.1 & 56 .\end{array}\right.$} & 11/14/83 & $\begin{array}{l}11 / \\
11 \\
12 / \\
12 / \\
1 /\end{array}$ \\
\hline 9 & $\begin{array}{l}3 / 6 \\
1 \\
135 / 137 \\
138 / 142 \\
11 / 12 \\
9 / 10 \\
5 a \\
7 b \\
5 b\end{array}$ & $\begin{array}{l}501 \\
614 \\
421 \\
423 \\
416 \\
422 \\
296 \\
299 \\
279\end{array}$ & $\begin{array}{l}1.1174 \\
1.9387 \\
0.7913 \\
0.7003 \\
0.9776 \\
0.9784 \\
0.7766 \\
0.7381 \\
0.6269\end{array}$ & $\begin{array}{l}1.1174 \\
1.9387 \\
0.7911 \\
0.7003 \\
0.9774 \\
0.9785 \\
0.7767 \\
0.7381 \\
0.6266\end{array}$ & $\begin{array}{l}0.0000 \\
0.0000 \\
0.0002 \\
0.0000 \\
0.0002 \\
+.0001 \\
+.0001 \\
0.0000 \\
0.0003\end{array}$ & $\begin{array}{rr}0.3 & 7 . \\
0.3 & 14 . \\
0.3 & 28 . \\
0.3 & 28 . \\
0.3 & 28 . \\
0.3 & 56 . \\
0.3 & 91 . \\
0.3 & 91 . \\
0.3 & 182 .\end{array}$ & $2 / 08 / 84$ & $\begin{array}{c}11 / 21 / 83 \\
11 / 28 / 83 \\
\vdots \\
12 / 12 / 83 \\
1 / 17 / 84 \\
5 / 09 / 84 \\
8 / 08 / 84\end{array}$ \\
\hline & $\begin{array}{l}\text { BLANK } \\
\text { BLANK } \\
\text { BLANK } \\
\text { BLANK } \\
\text { BLANK } \\
\text { BLANK }\end{array}$ & & & & & $\begin{array}{l}7 . \\
14 . \\
28 . \\
28 . \\
56 . \\
91 . \\
182 .\end{array}$ & $\begin{array}{c}1 / 19 / 84 \\
\vdots \\
2 / 08 / 84 \\
2 / 08 / 84\end{array}$ & $\begin{array}{l}1 / 21 / 84 \\
1 / 18 / 84 \\
2 / 1 / 84 \\
2 / 1 / 84 \\
2 / 29 / 84 \\
5 / 09 / 84 \\
8 / 8 / 84\end{array}$ \\
\hline
\end{tabular}


TEST III

J-13 MATER EXPERIMENT WITH TUFF

DUPF GLASS (MONOLITH)

\begin{tabular}{|c|c|c|c|c|c|c|}
\hline $\begin{array}{l}\text { No. } \\
\text { NT(g) }\end{array}$ & $\operatorname{IMITINL}_{\text {WEIGHT }}$ & $\begin{array}{l}\text { FINN } \\
\text { SEIGHT }\end{array}$ & $\begin{array}{r}\text { LEA } \\
\text { LOSS } 16 \\
\end{array}$ & $\begin{array}{l}T \\
\text { OSS( }(x)\end{array}$ & $\begin{array}{c}\text { FINLL } \\
\text { PHI }\end{array}$ & $\begin{array}{l}\text { Rock } \\
i \pi(0)\end{array}$ \\
\hline $\begin{array}{l}29.80 \\
25.20 \\
21.00 \\
30.50 \\
35.50\end{array}$ & $\begin{array}{l}115.11 \\
110.25 \\
105.85 \\
116.15 \\
121.56\end{array}$ & $\begin{array}{l}114.05 \\
109.51 \\
104.99 \\
114.85 \\
119.63\end{array}$ & $\begin{array}{l}1.06 \\
0.74 \\
0.86 \\
1.30 \\
1.93\end{array}$ & $\begin{array}{l}3.93 \\
3.30 \\
4.35 \\
4.70 \\
6.20\end{array}$ & $\begin{array}{l}9.21 \\
9.04 \\
9.26 \\
9.25 \\
9.25\end{array}$ & $\begin{array}{l}1.2 \\
1.0 \\
1.5 \\
1.7\end{array}$ \\
\hline $\begin{array}{r}16.70 \\
20.50 \\
14.01 \\
14.10 \\
13.90 \\
14.10 \\
9.87 \\
9.63 \\
9.28\end{array}$ & $\begin{array}{r}100.50 \\
106.99 \\
97.40 \\
98.23 \\
97.79 \\
97.83 \\
110.11 \\
109.80 \\
109.71\end{array}$ & $\begin{array}{r}99.82 \\
105.60 \\
95.90 \\
95.29 \\
97.06 \\
88.74 \\
109.78 \\
109.45 \\
109.14\end{array}$ & $\begin{array}{l}0.68 \\
1.39 \\
1.50 \\
2.94 \\
0.73 \\
9.09 \\
0.33 \\
0.35 \\
0.57\end{array}$ & $\begin{array}{r}7.14 \\
8.01 \\
13.03 \\
29.35 \\
6.19 \\
9.52 \\
3.46 \\
3.63 \\
6.18\end{array}$ & $\begin{array}{l}9.08 \\
9.37 \\
9.40 \\
9.50 \\
9.22 \\
9.55 \\
9.26 \\
9.30 \\
9.30\end{array}$ & $\begin{array}{l}1.0 \\
0.7 \\
0.7 \\
0.7 \\
0.7 \\
0.4 \\
0.5 \\
0.4\end{array}$ \\
\hline $\begin{array}{l}19.88 \\
20.01 \\
19.99 \\
20.00 \\
20.00 \\
20.00 \\
20.00\end{array}$ & $\begin{array}{r}99.56 \\
99.71 \\
99.56 \\
99.84 \\
99.66 \\
117.68 \\
118.21\end{array}$ & $\begin{array}{r}99.35 \\
97.94 \\
97.20 \\
97.96 \\
98.26 \\
117.34 \\
118.11\end{array}$ & $\begin{array}{l}0.21 \\
1.77 \\
2.46 \\
0.88 \\
1.04 \\
0.34 \\
0.10\end{array}$ & $\begin{array}{r}1.11 \\
10.69 \\
14.95 \\
4.98 \\
5.94 \\
1.73 \\
1.00\end{array}$ & $\begin{array}{l}9.02 \\
9.35 \\
9.77 \\
9.75 \\
9.55 \\
8.75 \\
8.44\end{array}$ & $\begin{array}{l}1 . \\
1 . \\
1 . \\
1 . \\
1 . \\
1 . \\
1 .\end{array}$ \\
\hline
\end{tabular}




$$
\text { TEST - III }
$$

J-13 MATER EXPERIMENT WITH TUFF

DWPF GLASS (MONOLITH)

DAY 7 - ICP Analys is

SAMLES

$\underline{S A / Y=0.3 \quad 0.5} \quad$ BLANK

\section{ELEM DET. LIM.}

$\begin{array}{ll}\text { Al } & .020 \\ \text { B } & .020 \\ \text { Fe } & .004 \\ \text { LI } & .001 \\ \text { Mn } & .0005 \\ \mathrm{NI} & .008 \\ \text { S1 } & .008 \\ \text { Sr } & .012 \\ \text { Zr } & .002 \\ \text { Ca } & .020 \\ \text { K } & .536 \\ \text { Mg } & .004 \\ \text { Ma } & .024\end{array}$

9

\subsection{7}

0.223

0.167

0.285

0.013

0.010

48.7

0.059

M

11.1

13.8

0.083

54.6
22

1.27

0.178

0.079

0.045

0.005

0.011

39.7

0.056

$\mathrm{MA}$

11.7

13.3

0.104

50.1

NOTE: Detection limit is 4 times the standard deviation of the blank.

Results are in micrograns per millititer (or ppm). M - elemental analysis not available. 
TEST - III

\section{J-13 WATER EXPERIMENT HITH TUFF}

DWP̈̈ GLASS (MONOLITH)

DAY 14 - ICP Analys is

SAMPLES

$S N / V=0.3$

0.5

BLANK

\section{ELEM DET. LIM.}

6

10

18

$\begin{array}{lllll}\text { A1 } & .020 & 1.13 & 1.14 & .936 \\ \mathrm{~B} & .020 & .218 & .280 & .201 \\ \mathrm{Fe} & .004 & .146 & .317 & .060 \\ \mathrm{LI} & .001 & .149 & .448 & .048 \\ \mathrm{Mn} & .0005 & .004 & .004 & .002 \\ \mathrm{Ni} & .008 & .017 & .024 & .004 \\ \mathrm{S1} & .008 & 46.3 & 52.5 & 51.0 \\ \mathrm{Sr} & .012 & .058 & .052 & .033 \\ \mathrm{Zr} & .002 & \mathrm{MA} & \mathrm{MK} & \mathrm{Mh} \\ \mathrm{Ca} & .020 & 8.88 & \mathrm{B.53} & 9.22 \\ \mathrm{~K} & .536 & 14.1 & 13.0 & 12.1 \\ \mathrm{Mg} & .0004 & .033 & .022 & .034 \\ \mathrm{Ma} & .024 & 51.7 & 54.5 & 56.1\end{array}$

Note: MA - elemental analys is not avallable. 


\section{TEST - III}

J-13 WATER EXPERIMENT WITH TUFF

DUPF GLASS (MOHOLITH)

DAY 28 - ICP Analysis

SAPLES

$\begin{array}{llllll}S A / V=0.1 & 0.3 & 0.3 & 0.2 & 0.3\end{array}$

BLAMKS

\begin{tabular}{lcccccccc} 
ELEM & DET LIM & 1 & 2 & 3 & 7 & 11 & 19 & 20 \\
\hline Al & .020 & 1.51 & 4.25 & .960 & .933 & .932 & 1.25 & 3.10 \\
$\mathrm{~B}$ & .020 & .206 & .244 & .333 & .219 & .251 & 0.209 & 0.205 \\
$\mathrm{Fe}$ & .004 & .112 & .311 & .088 & .479 & .387 & 0.080 & 0.185 \\
$\mathrm{Lf}$ & .001 & .155 & .394 & .406 & .208 & .499 & 0.051 & 0.049 \\
$\mathrm{Mn}$ & .00105 & .007 & .024 & .006 & .002 & .005 & 0.006 & 0.017 \\
$\mathrm{MI}$ & .0018 & .012 & .033 & .014 & .020 & .021 & 0.009 & 0.038 \\
$\mathrm{Si}$ & .008 & 46.5 & 61.5 & 63.4 & 48.1 & 48.4 & 58.9 & 59.2 \\
$\mathrm{Sr}$ & .012 & 0.055 & 0.057 & 0.058 & 0.037 & 0.035 & 0.034 & 0.042 \\
$\mathrm{Zr}$ & .002 & $\mathrm{MA}$ & $\mathrm{MA}$ & $\mathrm{MA}$ & $\mathrm{MA}$ & $\mathrm{MA}$ & $\mathrm{MA}$ & $\mathrm{NA}$ \\
$\mathrm{Ca}$ & .020 & $\mathbf{8 . 0 3}$ & $\mathbf{8 . 9 9}$ & 9.67 & 7.68 & 7.39 & 8.46 & 8.37 \\
$\mathrm{~K}$ & .536 & 12.2 & 14.7 & 13.3 & 11.5 & 11.5 & 16.8 & 14.7 \\
$\mathrm{Mg}$ & .004 & .053 & .080 & .030 & .023 & .027 & 0.047 & 0.083 \\
$\mathrm{Ma}$ & .024 & 52.0 & 59.0 & 65.0 & 54.0 & 55.1 & 59.6 & 56.2
\end{tabular}

Mote: $M A$ - elemental analysis not avallable. 


\section{TEST - IIJ}

J-13 HATER EXPERIMENT HITH TUFF

DUPF GLASS (MONOLITH)

DAY 56 - ICP Analys is

\section{SAMPLES}

$S N Y=0.1 \quad 0.3$

BLAMK

ELEM DET LIM

$12^{(1)}$

21

$\begin{array}{lllll}\text { A1 } & .020 & 0.783 & 0.190 & 2.55 \\ \text { B } & .020 & 0.222 & 0.705 & 0.211 \\ \text { Fe } & .004 & 0.039 & 0.210 & 0.177 \\ \text { L1 } & .001 & 0.260 & 1.68 & 0.050 \\ \text { Mn } & .0005 & 0.002 & 0.003 & 0.014 \\ \text { NI } & .008 & <.008 & <.008 & 0.009 \\ \text { S1 } & .008 & 50.0 & 101 . & 57.6 \\ \text { Sr } & .012 & .0 .024 & 0.072 & 0.031 \\ \text { Zr } & .002 & <.002 & <.002 & <.002 \\ \text { Ca } & .020 & 6.54 & 16.6 & 6.91 \\ \text { K } & .536 & 10.9 & 25.0 & 14.6 \\ \mathrm{Mg} & .004 & 0.035 & 0.087 & 0.077 \\ \mathrm{Ka} & .024 & 55.5 & 150 . & 56.3\end{array}$

Note: (1) Leachant lost >10\%. 


$$
\text { TEST - III }
$$

J-13 WATER EXPERIMENT HITH TUFF

DWPF GLASS (MONOLITH)

DAY 91 - ICP Analysis

\section{SAPLES}

$S A / V=0.3$

ELEM DET LIM

$\begin{array}{lll}\text { Al } & .012 & .486 \\ \text { B } & .024 & .285 \\ \mathrm{Fe} & .003 & <.003 \\ \mathrm{LI} & .002 & .802 \\ \mathrm{Mn} & .0003 & <.0003 \\ \mathrm{N1} & .008 & <.008 \\ \mathrm{SI} & .020 & 56.2 \\ \mathrm{Sr} & .016 & .022 \\ \mathrm{Zr} & .002 & <.002 \\ \mathrm{Ca} & .004 & 7.09 \\ \mathrm{~K} & .820 & 15.9 \\ \mathrm{Mg} & .001 & .028 \\ \mathrm{Na} & .028 & 63.8\end{array}$

0.3 25

.509

.129

.291

$<.003$

.886

$<.0003$

<.008

57.2

$<.016$

$<.002$

4.92

22.9

.012

64.0

.211

<.003

.053

$<.008$

52.5

.020

$<.002$

5.68

20.2

.017

58.0
BLAHK

$<.0003$ 


$$
\text { TEST - III }
$$

J-13 NATER EXPERIMENT MITH TUFF

DWPF GLASS (MOMOLITH)

DAY 162 - ICP Analysis

SAMPLE

SNY 0.3

BLANK

ELEM DET LIM

26

28

$\begin{array}{llll}\text { Al } & .012 & .839 & .412 \\ \text { B } & .024 & .396 & .215 \\ \text { Fe } & .003 & .122 & .062 \\ \text { LI } & .002 & 1.13 & .054 \\ \text { Mn } & .0003 & .002 & .004 \\ \text { Mi } & .008 & .015 & .018 \\ \text { S1 } & .020 & 57.4 & 47.7 \\ \text { Sr } & .016 & .037 & .073 \\ \text { Zr } & .002 & <.002 & <.002 \\ \text { Ca } & .004 & 6.62 & 13.4 \\ \text { K } & .820 & 17.5 & 22.5 \\ \mathrm{Mg} & .001 & .069 & .456 \\ \mathrm{Ma} & .028 & 57.6 & 53.4\end{array}$


TEST III

J-13 WATER EXPERIMENT MITH TUFF

DUPF GLASS (MONOLITH)

RAN DATA TABULATION - LEACHATE MIL YSIS

DAY 7

Analytical Result

SAMPLE ID

5

$9-$

Start Experinent

Stop Interval

Inttlal $\mathrm{pH}$

Final PH

Diff (pH)
Spec SA

Volume (g)

SNY

Tenp $\left({ }^{\circ} \mathrm{C}\right)$

Inttial Weight(g)

Final Height(g)

Weight Loss(g)

Zleach Loss

Initial Spec wt (g)

Final Spec Wt (g)

Spec Wt Loss(g)

Rock Weight(g)

$\begin{array}{ll}11 / 14 / 83 & 11 / 14 / 83 \\ 11 / 21 / 83 & 11 / 21 / 83 \\ 7.23 & 7.23 \\ 9.21 & 9.08 \\ 1.98 & 1.85 \\ 298 . & 501 . \\ 29.80 & 16.70 \\ 0.1 & 0.3 \\ 90.0 & 90.0 \\ 115.11 & 100.50 \\ 114.05 & 99.82 \\ 1.06 & 0.68 \\ 3.56 & 4.07 \\ 0.4094 & 1.1174 \\ 0.4095 & 1.1174 \\ +0.0001 & 0.0000 \\ 1.490 & 0.835\end{array}$

CONSTITUENT AMLLYIS(mg/liter)

Analyt ical Result

SAMPLE ID

$\begin{array}{lll}\text { A1 } & 1.27 & 1.17 \\ \text { B } & 0.202 & 0.223 \\ \text { Fe } & 0.698 & 0.167 \\ \text { Li } & 0.130 & 0.285 \\ \text { Mn } & 0.005 & 0.013 \\ M & 0.052 & 0.010 \\ \text { Si } & 45.8 & 48.7 \\ \text { Sr } & 0.061 & 0.059 \\ \text { Zr } & M . & M \\ \text { Ca } & 10.3 & 11.1 \\ K & 12.5 & 13.8 \\ \text { Mg } & 0.062 & 0.083 \\ M & 52.1 & 54.6\end{array}$

Corrected for Blank

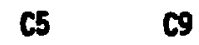

Mote: MA - elenental analysis not avallable. 
TEST III

J-13 WATER EXPERIMENT MITH TUFF

DUFF GLASS (MONOLITH)

RAH DATA TABULATION - LEACHATE ANALYSIS

DAY 14

Analytical Result

SPEC 10

6

10

Start Experiment

$11 / 14 / 83$

$11 / 14 / 83$

Stop Interval

Initlal pH

Final pH

$11 / 28 / 83$

7.23

9.04

Diff $\left.(\mathrm{pH}) \mathrm{Sm}^{2}\right)$

volume (g)

SNY

Temp $\left({ }^{\circ} \mathrm{C}\right)$

1.81

252.

25.20

0.1

90.0

Initial Height(g)

Final Helght $(g)$

Weight Loss(g)

ZLeach Loss

110.25

109.51

0.74

2.94

$11 / 28 / 83$

7.23

9.37

2.14

614.

20.50

Initfal Spec Wt (g)

Final Spec Ht (g)

Spec Wt Loss(g)

0.6230

0.3

90.0

105.99

105.60

1.39

6.78

0.6230

1.9387

0.0000

1.9385

Rock Welgint(g)

1.260

0.0002

1.025

CONSTITUENT ANH YSIS(mg/liter)

Analytical Result Corrected for Blank

SPEC ID

6

10

C6

C10

A)

1.13

0.218

1.14

0.280

0.146

0.317

0.149

0.448

0.004

0.004

0.017

0.024

46.3

52.5

0.058

0.052

Zr

$M$

M

Ca

8.8B

8.53

K

ing

14.1

13.0

0.033

0.022

Ma

51.7

54.5

$\begin{array}{ll}0.194 & 0.204 \\ 0.017 & 0.079 \\ 0.086 & 0.257 \\ 0.101 & 0.400 \\ 0.002 & 0.002 \\ 0.013 & 0.020 \\ -4.7 & 1.5 \\ 0.019 & 0.013 \\ M A & M 1 \\ -.34 & -.69 \\ 2.0 & 0.9 \\ -.001 & -.012 \\ -4.4 & -1.6\end{array}$

Hote: $M$ - elemental analys is not avaflabie. 
TEST III

J-13 MATER EXPERIMENT MITH TUFF

DWPF GLASS (MONOLITH)

RAH DATA TABULATION - LEACHATE ARALYSIS

DAY 28 (PAGE 1)

Analytical Result

\begin{tabular}{|c|c|c|}
\hline MPLE ID & 1 & 7 \\
\hline 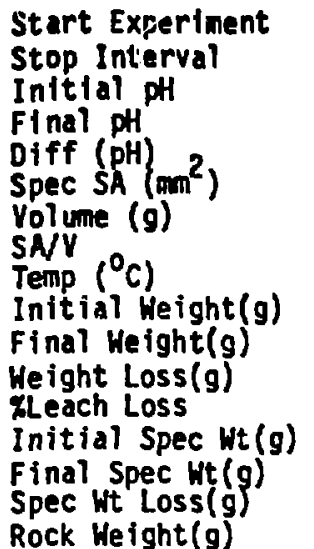 & $\begin{array}{l}11 / 14 / 83 \\
12 / 12 / 83 \\
7.23 \\
9.26 \\
2.03 \\
212 . \\
21.00 \\
0.1 \\
90.0 \\
105.85 \\
104.99 \\
0.86 \\
4.10 \\
0.3920 \\
0.3919 \\
0.0001 \\
1.050\end{array}$ & $\begin{array}{l}11 / 14 / 83 \\
12 / 12 / 83 \\
7.23 \\
9.25 \\
2.02 \\
305 . \\
30.50 \\
0.1 \\
90.0 \\
116.15 \\
114.85 \\
1.30 \\
4.26 \\
0.8025 \\
0.8024 \\
0.0001 \\
1.525\end{array}$ \\
\hline
\end{tabular}

\section{CONSTITUENT ANALYSIS(mg/liter)}

Analytical Result

Corrected for Blank

SAMPLE ID

1

7

$\mathrm{COI}$

$\mathrm{CO}$

$\begin{array}{lllll}\text { A1 } & 1.51 & 0.933 & -.665 & -1.242 \\ \text { B } & 0.206 & 0.219 & -.001 & -012 \\ \text { Fe } & 0.112 & 0.479 & -.021 & 0.346 \\ \text { Li } & 0.155 & 0.208 & 0.105 & 0.158 \\ \text { Mn } & 0.007 & 0.002 & -.005 & -.010 \\ \text { Ni } & 0.012 & 0.020 & -.012 & -.004 \\ \text { Si } & 46.5 & 48.1 & -12.6 & -11.1 \\ \text { Sr } & 0.055 & 0.037 & 0.017 & -.001 \\ \text { Zr } & \text { MA } & \text { NA } & \text { MA } & \text { MA } \\ \text { Ca } & 8.03 & 7.68 & -0.39 & -0.74 \\ \text { K } & 12.2 & 11.5 & -3.55 & -4.25 \\ \mathrm{Mg} & 0.053 & 0.023 & -.012 & -.042 \\ \mathrm{Ha} & 52.0 & 54.0 & -5.9 & -3.9\end{array}$

Hote: NA - elemental analysis not available. 
TEST III

J-13 WATER EXPERIMENT MITH TUFF

DNPF GLASS (MOHOLITH)

RAH DATA TABULATIOH, - LEACHATE MHLYSIS

DAY 28 (PAGE 2)

Analytical Result

SAPLE ID

Start Experfaent

Stop Interval

Initial pH

Final PH

Diff (pH) 2

Volume (g)

SNV

Teap $\left({ }^{\circ} \mathrm{C}\right)$

Inttral Weight(g)

Final Weight $(g)$

Neight Loss(g)

KLeach Loss

Inftial Spec Wt(g)

Final Spec Wt $(g)$

Spec Wt Loss(g)

Rock Weight (g)
2

11/14/83

$12 / 12 / 83$

7.23

9.40

2.17

421.

14.01

0.3

90.0

97.40

95.90

1.50

10.71

0.7913

0.7911

0.0002

0.700

3

11

$11 / 14 / 83 \quad 11 / 14 / 83$

$12 / 12 / 8312 / 1 c / 83$

$7.23 \quad 7.23$

$9.50 \quad 9.22$

$2.27 \quad 1.99$

423.416.

$14.10 \quad 13.90$

$0.3 \quad 0.3$

$90.0 \quad 90.0$

$98.23 \quad 97.79$

$95.29 \quad 97.06$

$2.94 \quad 0.73$

$20.85 \quad 5.25$

0.70030 .9776

$0.7003 \quad 0.9774$

$0.0000 \quad 0.0002$

$\begin{array}{ll}0.705 & 0.700\end{array}$

CONSTITUENT ANALYSIS(mg// Iter)

Analytical Result

SAMPLE ID

02

03

11

0.033

61.5

0.057

M

8.99

14.7

0.080

59.0
0.932

0.251

0.387

0.499

0.005

0.021

$\begin{array}{ll}0.960 & 0.932 \\ 0.333 & 0.251 \\ 0.088 & 0.387 \\ 0.406 & 0.499 \\ 0.006 & 0.005 \\ 0.014 & 0.021 \\ 63.4 & 48.1 \\ 0.058 & 0.035 \\ 14 & 14 \\ 9.67 & 7.39 \\ 13.3 & 11.5 \\ 0.030 & 0.027 \\ 65.0 & 55.1\end{array}$

$65.0 \quad 55.1$
Corrected for Blank

$\mathrm{CO} 2 \mathrm{CO} \quad \mathrm{Cl}$

$\begin{array}{lll}2.42 & -0.87 & -0.90 \\ 0.037 & 0.126 & 0.044 \\ 0.178 & -0.025 & 0.254 \\ 0.344 & 0.356 & 0.449 \\ -0.012 & -0.006 & -0.007 \\ 0.009 & -0.010 & -0.003 \\ 2.3 & 4.3 & -10.8 \\ 0.019 & 0.020 & -0.003 \\ M & M & M \\ 0.39 & 0.75 & -0.97 \\ -1.05 & -2.45 & -4.25 \\ 0.015 & -0.035 & -.038 \\ 1.1 & 7.1 & -2.8\end{array}$

Note: $M$ - elemental analys is not avaflable. 
TEST III

J-13 HATER EXPERIMENT WITH TUFF

DUPF GLASS (MONOLITH)

RAW DRTA TABULATION - LEACHRTE MUALYSIS

DAY 56

Analyt lcal Result

SNPLE ID

8

12

Start Experiment

Stop interval

Initial pH

Final pH

Diff (pH) ${ }^{2}$ )

Yolume ( $(9)$

SNY

Temp $\left({ }^{\circ} \mathrm{C}\right)$

Inttial Weight(g)

Final Weight $(g)$

Height Loss $(g)$

KLeach Loss

Initial Spec Wt (g)

Final Spec Wt(g)

Spec Wt Loss (g)

Rock Height (g)

$11 / 14 / 83 \quad 11 / 14 / 83$

$1 / 17 / 84 \quad 1 / 17 / 84$

$7.23 \quad 7.23$

$9.25 \quad 9.55$

$2.02 \quad 2.32$

355. 422.

$35.50 \quad 14.10$

$0.1 \quad 0.3$

$90.0 \quad 90.0$

$121.56 \quad 97.83$

119.6388 .74

$1.93 \quad 9.09$

$5.44 \quad 64.46$

$0.9009 \quad 0.9784$

0.90010 .9785

$0.0008+0.0001$

$1.775 \quad 0.705$

CONSTITUENT ANAL YSIS (mg/l fter)

Analytical Result

Corrected for Blank

SAMPLE ID

8

12

$-.-$

C8

$\mathrm{C12}$

$\begin{array}{lll}\text { A1 } & 0.783 & 0.190 \\ \text { B } & 0.222 & 0.705 \\ \text { Fe } & 0.039 & 0.210 \\ \text { Li } & 0.260 & 1.68 \\ \text { Mn } & 0.002 & 0.003 \\ \text { Mi } & 5.008 & <.008 \\ \text { Si } & 50.0 & 101 . \\ \text { Sr } & 0.024 & 0.072 \\ \text { Zr } & <0.002 & <0.002 \\ \text { Co } & 5.00 & 16.6 \\ \text { K } & 10.9 & 25.0 \\ \text { Mg } & 0.035 & 0.087 \\ \text { Ma } & 55.5 & 150 .\end{array}$

$\begin{array}{ll}-1.767 & -2.36 \\ 0.011 & 0.494 \\ -0.138 & 0.023 \\ 0.210 & 1.18 \\ -0.012 & -0.011 \\ <.008 & <.008 \\ -7.6 & 43.4 \\ -0.007 & 0.041 \\ <0.002 & <0.002 \\ -0.37 & 6.97 \\ -3.7 & 10.4 \\ -0.042 & 0.010 \\ -0.80 & 93.7\end{array}$


TEST 1 II

J-13 WATER EXPERIMENT HITH TUFF

DHPF GLASS (MONOLITH)

RAW DATA TABULATION - LEACHATE ANALYSIS

DAY 91

Analytical Result

SAPLE ID

24

25

Start Experiment

Stop Interval

Inftial pH

Final pH

Diff (pH) ${ }_{\text {Spec } S A}^{2}$ )

Volume (g)

SAY $\left({ }^{\circ} \mathrm{C}\right)$

Initiar Neight(g)

Final Weight $(g)$

Weight Loss(g)

ZLeach Loss

Initial Spec Wt (g)

Final Spec Ht $(g)$

Spec Wt Loss(g)

Rock Welght $(g)$

$11 / 14 / 83 \quad 11 / 14 / 83$

$5 / 9 / 84 \quad 5 / 9 / 84$

$7.23 \quad 7.23$

$9.26 \quad 9.30$

$2.03 \quad 2.07$

$296.1 \quad 299.4$

$9.87 \quad 9.98$

$0.3 \quad 0.3$

$90.0 \quad 90.0$

$110.11 \quad 109.80$

$109.78 \quad 109.45$

$0.33 \quad 0.35$

$3.0 \quad 2.8$

$0.7766 \quad 0.7381$

$\begin{array}{ll}0.7767 & 0.7381\end{array}$

$+0.0001 \quad 0.0000$

$0.495 \quad 0.500$

CONSTITUENT ANALYSIS(mg/lfter)

Analytical Result

SAPPLE ID

Al

B

Fe

11

Pin

Ni

Si

Sr

Zr

Ca

K

Mg

Ma
24 (d) 25 (d)

$.486(<.028) \quad .509(<.028)$

$.285(0.60) \quad .291(0.50)$

$<.003$

$.802(.50)$

$<.0003$

$<.008$

$56.2(48$.

0.022 .

$\$ .002$

7.09

15.9

.028 ( .001 )

63.8(50.)
$<.003$

$.886(.70)$

$<.0003$

<.008

57.2(49.)

$<0.016$

$<0.002$

4.92

22.9

$.012(<.001$

64.0(53.)
Corrected for Blank

C24 (d) C25 (d)

$.357(<-) \quad .380(<-)$

$.074(.39) \quad .080(.29)$

$.749(.50) \quad .833(.70)$

$<.0003<.0003$

$<.008<.008$

3.7(-1.) 4.7 $(0.0)$

$0.002<0.016$

$<0.002<0.002$

$1.41 \quad-.76$

$-4.3 \quad 2.7$

$.011(<-) \quad-.005(<-)$

$5.8(-1.0) \quad 6.0(+2$.

NOTE: Values in parentheses were analyzed on another instrument. 
TEST III

J-13 WATER EXPERIMENT WITH TUFF

DUPF GLASS (MONOLITH)

RAW DATA TABULATION - LEACHATE AHLYYSIS

DAY 182

Analytical Result

SAPLE ID

26

Start Experiment

Stop Interval

Initial pH

Final pH

Diff (pH)

Spec SA $\left(\operatorname{mon}^{2}\right)$

Volume (g)

SNY

Tenp ${ }^{\circ} \mathrm{C}$

Initial Weight $(g)$

Final Height $(g)$

Weight Loss(g)

zLeach Loss

$2 / 8 / 84$

$8 / 08 / 84$

7.23

9.30

2.07

278.6

9.285

0.3

90.0

109.71

109.14

Initial Spec $W($ (g)

0.57

6.18

Final Spec it(g)

Spec Wt Loss $(g)$

0.6269

Rock Weight $(g)$

0.6266

0.0003

0.465

CONSTITUENT ARALYSIS(mg/litEr)

Analytical Result Corrected for Blank

SAMPLE ID

26

C26
A1
0.839
0.427
0.396
0.122
0.181
Fe
1.13
0.060
LI
0.002
1.08
Mn
0.015
57.4
0.037
$-.002$
Ni
$<0.002$
$-.003$
9.7
Sr
6.62
17.5
0.069
$-0.036$
$<0.002$
$\mathrm{Ca}$
Mg
57.6
$-6.8$
$-5.0$
$-.387$
Na
4.2

Mote: Used Parr Bombs 


\section{Appendix E. \\ Test IV: DWPF Glass-J-13 Water + Tuff + Stainless Steel Experiment, Monolith Samples}




\section{TEST IV}

J-13 MATER EXPERIMEMT MITH TUFF AKD STAIMLESS STEEL

DUPF GLASS (MOMOLITH)

\begin{tabular}{|c|c|c|c|c|c|c|c|c|c|}
\hline & $\begin{array}{l}\text { GLAss } \\
\text { No. }\end{array}$ & $\left(\mathrm{m}^{2}\right)$ & $\begin{array}{l}T I A L \\
S(0)\end{array}$ & $\operatorname{FIm}_{\operatorname{MSS}(g)}$ & $\begin{array}{l}\text { MSSS } \\
\text { LOSS(0) }\end{array}$ & SNY & $\begin{array}{l}\text { INTERYAL } \\
\text { TIME (d) }\end{array}$ & $\begin{array}{l}\text { TIMEE } \\
\text { START } \\
\end{array}$ & $\begin{array}{l}\text { TIME } \\
\text { STOP } \\
\end{array}$ \\
\hline & $\begin{array}{l}35+36 \\
14+37 \\
16+38 \\
39 \\
22+23 \\
24+25 \\
27+29 \\
26+33 \\
32\end{array}$ & $\begin{array}{l}404 . \\
439 . \\
458 . \\
364 . \\
440 . \\
408 . \\
464 . \\
452 . \\
323 .\end{array}$ & $\begin{array}{l}1.0470 \\
1.0521 \\
1.0816 \\
1.7904 \\
1.1358 \\
0.9309 \\
1.0007 \\
0.8545 \\
0.9263\end{array}$ & $\begin{array}{l}1.0467 \\
1.0520 \\
1.0817 \\
1.7903 \\
1.1355 \\
0.9315 \\
1.0008 \\
0.8549 \\
0.9264\end{array}$ & $\begin{array}{l}0.0003 \\
0.0001 \\
0.0001+ \\
0.0001 \\
0.0003 \\
0.0006+ \\
0.0001+ \\
0.0004+ \\
0.0001+\end{array}$ & $\begin{array}{l}.3 \\
.3 \\
.3 \\
.3 \\
.3 \\
.3 \\
.3\end{array}$ & $\begin{array}{r}3 \\
7 \\
14 \\
28 \\
28 \\
57 \\
91 \\
91 \\
182\end{array}$ & $\begin{array}{c}5 / 07 / 84 \\
\vdots \\
\vdots \\
\vdots\end{array}$ & $\begin{array}{l}5 / 10 / 84 \\
5 / 14 / 84 \\
5 / 21 / 84 \\
6 / 04 / 84 \\
7 / 03 / 84 \\
8 / 06 / 84 \\
11 / 5 / 84\end{array}$ \\
\hline
\end{tabular}

\begin{tabular}{|c|c|c|c|c|c|c|}
\hline $\begin{array}{ll}10 & 30 \\
11 & 31 \\
12 & 40 \\
13 & 41 \\
14 & 42 \\
15 & 43 \\
16 & 44 \\
17 & 45 \\
18 & 21+28+34\end{array}$ & $\begin{array}{l}564: \\
591 . \\
584 . \\
583 . \\
599 . \\
597 . \\
618 . \\
615 . \\
435 .\end{array}$ & $\begin{array}{l}1.5204 \\
2.0428 \\
1.3456 \\
1.4772 \\
1.2733 \\
1.4150 \\
1.6354 \\
2.7131 \\
0.8719\end{array}$ & $\begin{array}{l}1.5205 \\
2.0429 \\
1.3434 \\
1.4771 \\
1.2734 \\
1.4153 \\
1.6363 \\
2.7133 \\
0.8722\end{array}$ & $\begin{array}{l}0.0001+ \\
0.0001 t \\
0.0022 \\
0.0001 \\
0.0001+ \\
0.0003+ \\
0.0009+ \\
0.0002 \\
0.0003+\end{array}$ & $\begin{array}{r}3 \\
7 \\
14 \\
28 \\
28 \\
57 \\
91 \\
91 \\
182\end{array}$ & $\begin{array}{ll}* & 5 / 10 / 84 \\
* & 5 / 14 / 84 \\
* & 5 / 21 / 84 \\
* & 6 / 04 / 84 \\
* & 7 / 03 / 84 \\
* & 8 / 06 / 84 \\
* & 11 / 5 / 84\end{array}$ \\
\hline $\begin{array}{ll}19 & \text { BLANK } \\
20 & \text { BLANK } \\
21 & \text { BLANK } \\
22 & \text { BLANK } \\
23 & \text { BLANK } \\
24 & \text { BLANK } \\
25 & \text { BLANK } \\
26 & \text { BLANK } \\
27 & \text { BLANK }\end{array}$ & $\begin{array}{l}- \\
- \\
- \\
- \\
- \\
-\end{array}$ & & & & $\begin{array}{r}3 \\
7 \\
14 \\
28 \\
28 \\
57 \\
91 \\
91 \\
182\end{array}$ & $\begin{array}{ll} & 5 / 10 / 84 \\
* & 5 / 14 / 84 \\
* & 5 / 21 / 84 \\
* & 6 / 04 / 84 \\
* & 7 / 03 / 84 \\
* & 8 / 06 / 84 \\
-\quad & 11 / 5 / 84\end{array}$ \\
\hline
\end{tabular}


TEST IV

J-13 WATER EXPERIMENT HITH TUFF AND STAINLESS STEEL

DWPF GLASS (MONOLITH)

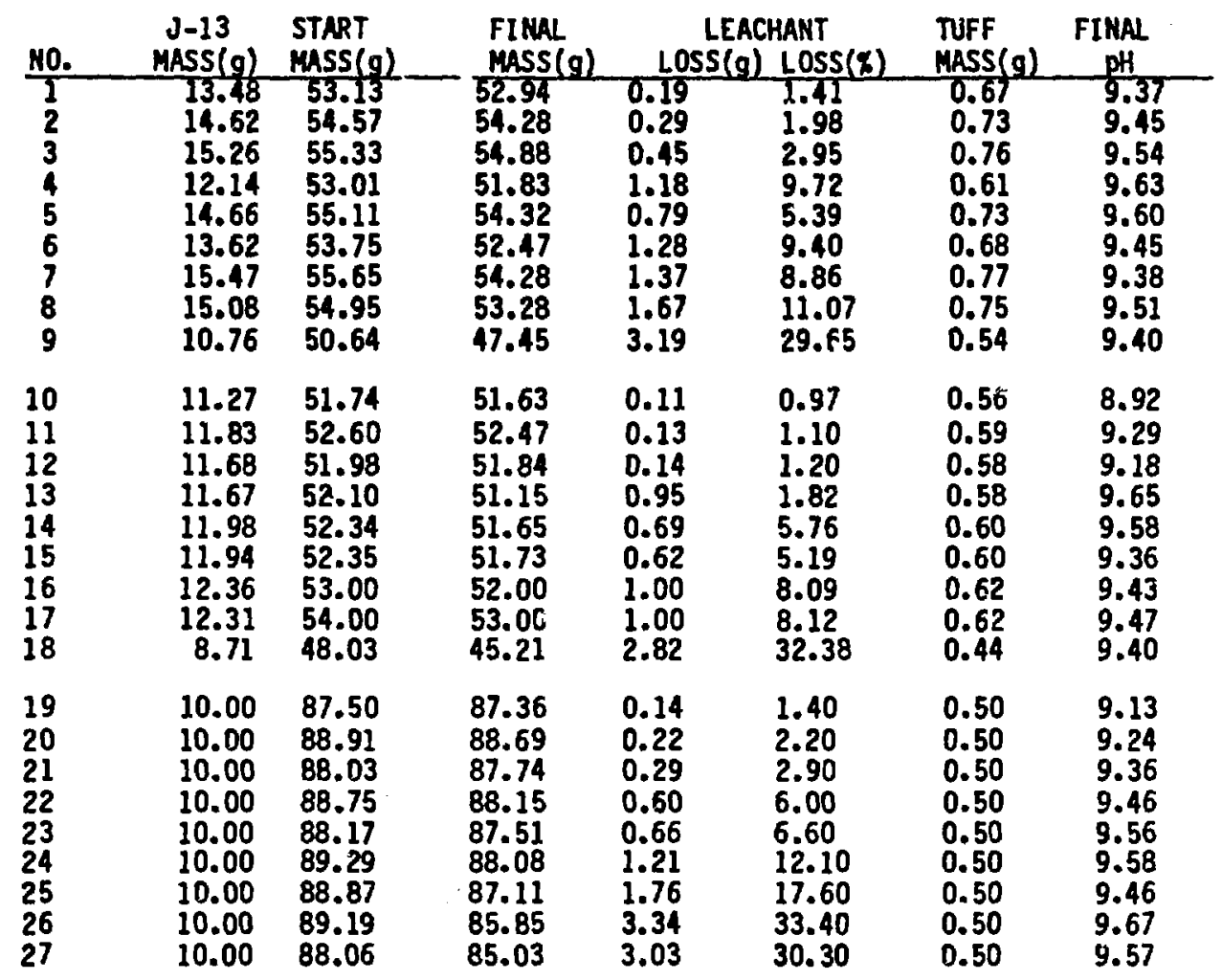


TEST IV

J-13 MATER EXPERIMENT HITH TUFF AND STAIALLSS STEEL

DWPF GLASS (MONOITH)

DAY 3 - ICP Analysis

SAMPLES

$\underline{S A Y V=0.3 \quad 0.5 \quad \text { BLANK } \quad \text { J.13 }}$

ELEM DET. LIM.

10

19

$\begin{array}{llllll}\text { Al } & .012 & 1.60 & .628 & .580 & .677 \\ \mathrm{~B} & .024 & .280 & .289 & .273 & .240 \\ \mathrm{Fe} & .003 & .697 & .048 & .240 & .021 \\ \mathrm{LI} & . .002 & .120 & .281 & .046 & .043 \\ \mathrm{Mn} & .0003 & .010 & .002 & .004 & .003 \\ \mathrm{Ni} & .008 & .011 & <.008 & .011 & <.008 \\ \mathrm{Si} & .020 & 44.4 & 42.1 & 43.3 & 39.3 \\ \mathrm{Sr} & .016 & .036 & .030 & .033 & .036 \\ \mathrm{Zr} & .002 & .002 & .003 & <.002 & .004 \\ \mathrm{Ca} & .004 & 9.97 & 9.80 & 11.1 & 10.6 \\ \mathrm{~K} & .920 & 18.2 & 63.5 & 21.0 & 11.7 \\ \mathrm{Mg} & .001 & .341 & .084 & .612 & .149 \\ \mathrm{Ha} & .028 & 51.1 & 52.1 & 53.6 & 49.1\end{array}$




\section{TEST IV}

J-13 WATER EXPERIMENT HITH TUFF AND STAIRLESS STEEL

DHPF GLASS (MONOLITH)

DAY 7 - ICP Analysis

SAPLES

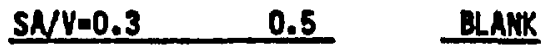

ELEM DET. LIM.

211

20

$\begin{array}{lllll}\text { Al } & .012 & 2.12 & .827 & .404 \\ \mathrm{~B} & .024 & .309 & .328 & .288 \\ \mathrm{Fe} & .003 & .118 & .171 & .043 \\ \mathrm{LI} & .002 & .256 & .417 & .047 \\ \mathrm{Mn} & .0003 & .015 & .005 & <.0003 \\ \mathrm{Mi} & .008 & .009 & <.008 & <.008 \\ \mathrm{Si} & .020 & 49.3 & 44.5 & 45.3 \\ \mathrm{Sr} & .016 & .045 & .040 & .040 \\ \mathrm{Zr} & .002 & <.002 & <.002 & <.002 \\ \mathrm{Ca} & .004 & 10.1 & 9.88 & 11.3 \\ \mathrm{~K} & .820 & 19.8 & 23.0 & 16.4 \\ \mathrm{Mg} & .001 & .075 & .058 & .065 \\ \mathrm{Ma} & .028 & 55.1 & 55.0 & 55.2\end{array}$




\section{TEST IV}

J-13 WATER EXPERINENT WITH TUFF AND STAIMLESS STEEL

DWPF GLASS (MONOLITH)

DAY 14 - ICP Analysis

SAPLES

$\underline{S A} / V=0.3 \quad 0.5 \quad$ BLAHK

ELEM DET. LIM.

3

12

21

$\begin{array}{lllll}\text { Al } & .012 & .755 & .460 & .464 \\ \text { B } & .024 & .348 & .388 & .289 \\ \text { Fe } & .003 & .045 & .170 & .117 \\ \text { L1 } & .002 & .422 & .739 & .048 \\ \text { Nn } & .0003 & .002 & .003 & .003 \\ \text { Ni } & .008 & <.008 & <.008 & <.008 \\ \text { Si } & .020 & 49.7 & 46.1 & 46.8 \\ \text { Sr } & .016 & .043 & .033 & .028 \\ \text { Zr } & .002 & 4.002 & 4.002 & <.002 \\ \text { Ca } & .004 & 9.69 & 10.1 & 9.96 \\ \text { K } & .820 & 19.7 & 26.1 & 17.1 \\ \mathrm{Mg} & .001 & .044 & .049 & .214 \\ \mathrm{Na} & .028 & 55.3 & 58.2 & 55.1\end{array}$




\section{TEST IV}

J-13 WATER EXPERIMENT WITH TLFF AND STAINLESS STEEL

DHPF GLASS (MONOLITH)

DAY $2 B$ - ICP Analysis

SAPLES

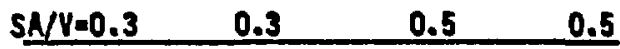

BLANKS

ELEM-D.L.

5

13

14

22

23

$\begin{array}{llllllll}\text { Al } & .012 & .679 & .613 & .588 & .673 & .543 & .495 \\ \mathrm{~B} & .024 & .353 & .375 & .423 & .435 & .329 & .316 \\ \mathrm{ie} & .003 & .039 & .031 & .051 & .051 & .037 & .017 \\ \mathrm{LI} & .002 & .053 & .524 & .852 & .946 & .049 & .049 \\ \mathrm{Mn} & .0003 & .002 & .001 & .003 & .006 & .002 & .001 \\ \mathrm{Mi} & .008 & <.008 & <.008 & <.008 & <.008 & <.008 & <.008 \\ \mathrm{Si} & .020 & 57.5 & 54.4 & 56.5 & 54.9 & 52.0 & 53.6 \\ \mathrm{Sr} & .016 & .032 & .033 & .015 & .025 & .022 & .025 \\ \mathrm{Zr} & .002 & <.002 & <.002 & <.002 & <.002 & <.002 & <.002 \\ \mathrm{Ca} & .004 & 9.29 & 9.25 & 8.12 & 8.54 & 10.3 & 8.93 \\ \mathrm{~K} & .820 & 41.0 & 21.6 & 26.1 & 29.5 & 20.6 & 19.8 \\ \mathrm{Mg} & .001 & .021 & .019 & .025 & .056 & .128 & .012 \\ \mathrm{Ha} & .028 & 61.3 & 58.5 & 63.2 & 62.2 & 57.8 & 58.4\end{array}$




\section{TEST IV}

J-13 MATER EXPERIMENT UITH TUFF AND STAIMLESS STEEL

DUPF GLASS (MONOLITH)

DAY 57- Icp Analysis

SAMLES

$\begin{array}{lll}S A / V=0.3 \quad 0.5 & \text { BLANK }\end{array}$

ELEM DET. LIM.

6

15

24

$\begin{array}{lllll}\text { Al } & .012 & .059 & .553 & .521 \\ \text { B } & .024 & .394 & .437 & .322 \\ \mathrm{Fe} & .003 & .028 & .039 & .018 \\ \mathrm{LI} & .002 & .547 & 1.08 & .056 \\ \mathrm{Mn} & .0003 & .003 & .002 & .003 \\ \mathrm{Hi} & .008 & .032 & <.008 & <.008 \\ \mathrm{Si} & .020 & 61.4 & 57.0 & 60.5 \\ \mathrm{Sr} & .016 & .070 & .068 & .061 \\ \mathrm{Ir} & .002 & .005 & <.002 & <.002 \\ \mathrm{Ca} & .004 & 9.39 & 7.88 & 7.55 \\ \mathrm{~K} & .820 & 30.2 & 37.1 & 18.2 \\ \mathrm{Mg} & .001 & .009 & .009 & .006 \\ \mathrm{Na} & .028 & 60.0 & 59.6 & 59.8\end{array}$




\section{TEST IV}

J-13 WATER EXPERIMENT WITH TUFF AND STAINLESS STEEL

DHPF GLASS (MONOLITH)

DAY 91- Icp Analysis

SNPLES

\begin{tabular}{llll} 
SNVM=0.3 & 0.3 & 0.5 & 0.5 \\
\hline
\end{tabular}

16

ELEM-D.L.

A1 $\quad 012$

.477

.713

B .024

.395

.409

Fe .003

.053

.077

.760

.003

Mn .0003 .001

Ni .008

$<.008$

$<.008$

Si .020

64.7

66.4

.054

Sr $\quad .016 \quad .055$

Zr .002

Ca .004

.003

.002

8.72

26.8

9.04

K .820

22.0

Hg .001

.042

Na $\quad .028$

60.8

.019

63.1

.501

.430

.015

1.18

.002

$<.008$

62.9

.047

.002

8.19

20.7

.013

62.7

.475

.998

.487

.445

.421

.441

.032

.035

.018

1.31

.063

.075

.002

.015

.003

$<.008$

.561

.014

62.5

67.5

82.9

.052

.062

.063

.002

.002

.002

8.91

10.6

10.6

20.7

20.5

22.7

.013

.013

.018

62.6

68.7

76.5 


\section{TEST IV}

J-13 WATER EXPERIMENT WITH TUFF AND STAINLESS STEEL

DWPF GLASS (MONOLITH)

RAW DATA TABULATION - LEACHATE AMLLYSIS

DAY 3

Anarytical Result

SAMPLE ID

1

10

Start Experiment

Stop Interval

Intial pH

Final $\mathrm{pH}$

Diff $\left.(\mathrm{pH}) \mathrm{m}^{2}\right)$

Volume (g)

SA/V

Temp $\left({ }^{\circ} \mathrm{C}\right)$

Initial Weight(g)

Final Weight(g)

He ight Loss (g)

Xleach Loss

Initial Spec Wt $(g)$

Final Sec Wt $(g)$

Spec Wt Loss(g)

Rock Weight (g)

$\begin{array}{ll}5 / 7 / 84 & 5 / 7 / 84 \\ 5 / 10 / 84 & 5 / 10 / 84 \\ 7.98 & 7.98 \\ 9.37 & 8.92 \\ 1.39 & 0.96 \\ 404 . & 564 . \\ 13.48 & 11.27 \\ 0.3 & 0.5 \\ 90.0 & 90.0 \\ 53.13 & 51.74 \\ 52.94 & 51.63 \\ 0.19 & 0.11 \\ 1.41 & 0.97 \\ 1.0470 & 1.5204 \\ 1.0467 & 1.5205 \\ 0.0003 & +0.0001 \\ 0.670 & 0.560\end{array}$

CONSTITUENT ANALYSIS(mg/liter)

Analytical Result

Corrected for Blank

SAMPLE ID

\section{1}

10

1.60

0.280

0.697

0.120

0.010

0.011

44.4

0.036

0.002

9.97

18.2

0.341

51.1
0.628

0.289

0.048

0.281

0.002

$<.008$

42.1

0.030

0.003

9.80

63.5

0.084

52.1
C1

C10

$\begin{array}{ll}1.02 & 0.048 \\ 0.007 & 0.016 \\ 0.457 & -0.192 \\ 0.074 & 0.235 \\ 0.006 & -.002 \\ 0.000 & <.008 \\ 1.1 & -1.2 \\ 0.003 & -.003 \\ 0.002 & 0.003 \\ -1.13 & -1.3 \\ -2.8 & 42.5 \\ -.271 & -.528 \\ -2.5 & -1.5\end{array}$


TEST IV

J-13 WATER EXPERIMENT WITH TUFF AND STAIRLESS STEEL

DUPF GLASS (MONOLITH)

RAH DATA TABULATION - LEACHATE AMLYSIS

DAY 7

Analytical Result

SAMPLE ID

2

11

Start Experiment

Stop Interval

Initial $\mathrm{pH}$

Final pH

Diff (pH)

Spec SA $\left(\mathrm{mm}^{2}\right)$

Volume $(g)$

SNV

Temp $\left({ }^{\circ} \mathrm{C}\right)$

Initial Weight(g)

Final Height $(g)$

Weight Loss (g)

XLeach Loss (g)

Initial Spec Wt (g)

Final Spec Wt (g)

Spec Ut Loss $(g)$

Rock Weight(g)

$5 / 7 / 84 \quad 5 / 7 / 84$

$5 / 14 / 84 \quad 5 / 14 / 84$

$7.98 \quad 7.98$

$9.45 \quad 9.29$

$1.47 \quad 1.31$

439.559.

$14.62 \quad 11.83$

$0.3 \quad 0.5$

$90.0 \quad 90.0$

$54.57 \quad 52.60$

$54.28 \quad 52.47$

$\begin{array}{ll}0.29 & 0.13\end{array}$

$1.98 \quad 1.10$

$1.0521 \quad 2.0428$

$1.0520 \quad 2.0429$

$0.00010 .0001+$

$\begin{array}{ll}0.73 & 0.59\end{array}$

CONSTITUENT ANALYSIS(ng/liter)

Anaiyt fcal Result

Corrected for slank

SNAPLE ID

2

11

C2

CII

$\begin{array}{lll}A 1 & 2.12 & 0.827 \\ \text { B } & 0.309 & 0.328 \\ \text { Fe } & 0.118 & 0.171 \\ \text { Li } & 0.256 & 0.417 \\ \text { Mn } & 0.015 & 0.005 \\ \text { Mi } & 0.009 & 2.008 \\ \text { Si } & 49.3 & 44.5 \\ \text { Sr } & 0.045 & 0.040 \\ \text { Zr } & 5.002 & 2.002 \\ \text { C. } & 10.1 & 9.88 \\ K & 19.8 & 23.0 \\ \text { Mg } & 0.075 & 0.058 \\ \text { M. } & 55.1 & 55.0\end{array}$

1.716
0.021
0.075
0.209
0.025
0.009
4.0
0.005
$<.002$
-1.2
3.4
0.010
-0.1

0.423

0.040

0.128

0.370

0.005

$<.008$

$-0.8$

0.000

5.002

$-1.4$

6.6

$-0.007$

$-0.2$ 
TEST IV

J-13 HATER EXPERIMENT MITH TUFF AHD STAIMLESS STEEL

DHPF GLASS (MOMOLITH)

RAH DATA TABULATIOH - LEACHATE AMALYSIS

DAY 14

Analytical Result

SAMPLE ID

$3 \quad 22$

Start Experiment

Stop Interval

Initial pH

Final $\mathrm{pH}$

Diff (pH)
Spec SA

volume (g)

SAN

Temp ( $\left.{ }^{\circ} \mathrm{C}\right)$

Inftial Weight(g)

Final Weight $(g)$

Height Loss $(g)$

ZLeach Loss

Initial Spec Wt(g)

Final Spec Wt (g)

Spec Wt Loss (g)

Rock Weight $(g)$

$\begin{array}{ll}5 / 7 / 84 & 5 / 7 / 84 \\ 5 / 21 / 84 & 5 / 21 / 84 \\ 7.98 & 7.98 \\ 9.54 & 9.18 \\ 1.56 & 1.20 \\ 458 . & 584 . \\ 15.26 & 11.68 \\ 0.3 & 0.5 \\ 90.0 & 90.0 \\ 55.33 & 51.98 \\ 54.88 & 51.84 \\ 0.45 & 0.14 \\ 2.95 & 1.20 \\ 1.0816 & 1.3456 \\ 1.0817 & 1.3434 \\ 0.0001+ & 0.0022 \\ 0.76 & n .58\end{array}$

CONSTITUENT ANALYSIS(mg/liter)

Analyt Ica? Result

Corrected for Blank

SANPLE ID

3

12

C3

C12

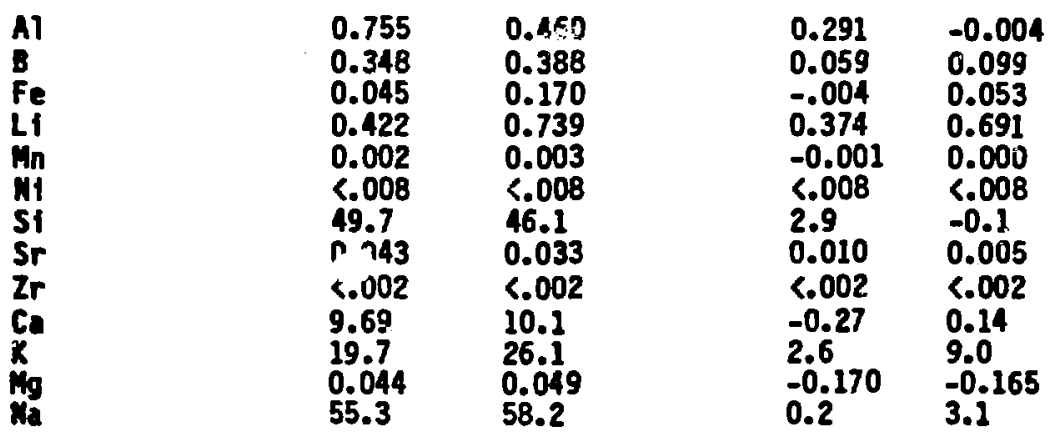


TEST IV

J-13 WATER EXPERIMENT WITH TUFF AND STAIMLESS STEEL

DUPF GLASS (MOHOLITH)

RAN DATA TABULATION - LEACHATE ANALYSIS

DAY 28 (PAGE 1)

Analytical Result

SAMFLE ID

Start Experiment

Stop Irite yal

Initial pil

Final pH

Diff $(\mathrm{pH}) \mathrm{m}^{2}$

Volune $(g)$

SA/V

Temp $\left({ }^{\circ} \mathrm{C}\right)$

Initial Weight(g)

Final Weight $(g)$

Height Loss(g)

ZLeach Loss

Initial Spec Ht(g)

Final Spec Wt $(g)$

Spec Wt Loss $(g)$

Rock Weight $(g)$
4

$\begin{array}{ll}5 / 7 / 84 & 5 / 7 / 84 \\ 6 / 4 / 84 & 6 / 4 / 84 \\ 7.98 & 7.98 \\ 9.63 & 9.60 \\ 1.74 & 1.62 \\ 364 . & 440 . \\ 12.14 & 14.66 \\ 0.3 & 0.3 \\ 90.0 & 90.0 \\ 53.01 & 55.11 \\ 51.83 & 54.32 \\ 1.18 & 0.79 \\ 9.72 & 5.39 \\ 1.7904 & 1.1358 \\ 1.7903 & 1.1355 \\ 0.0001 & 0.0003 \\ 0.61 & 0.73\end{array}$

CONSTITUENT ANALYSIS(mg/liter)
Analytical Result Corrected for Blank

\begin{tabular}{|c|c|c|c|c|}
\hline SAMPLE ID & 4 & 5 & $c 4$ & $C 5$ \\
\hline $\begin{array}{l}A 1 \\
B \\
\mathrm{Fe} \\
\mathrm{Li} \\
\mathrm{Hin} \\
\mathrm{Mi} \\
\mathrm{Si} \\
\mathrm{Sr} \\
\mathrm{Zr} \\
\mathrm{Ca} \\
\mathrm{Mg} \\
\mathrm{Ma}\end{array}$ & $\begin{array}{l}.679 \\
.353 \\
.039 \\
.053 \\
.002 \\
<.008 \\
57.5 \\
0.032 \\
6.002 \\
9.29 \\
.021 \\
61.3\end{array}$ & $\begin{array}{l}.613 \\
.375 \\
.031 \\
.524 \\
.001 \\
<.008 \\
54.4 \\
0.033 \\
<.002 \\
9.25 \\
.019 \\
58.5\end{array}$ & $\begin{array}{l}.160 \\
.030 \\
.011 \\
.004 \\
.000 \\
.0 .008 \\
5.0 \\
0.008 \\
<.002 \\
. .3 \\
.009 \\
3.2\end{array}$ & $\begin{array}{l}.094 \\
.052 \\
.004 \\
.475 \\
.000 \\
5.008 \\
1.9 \\
0.009 \\
<.002 \\
. .40 \\
.007 \\
.4\end{array}$ \\
\hline
\end{tabular}


TEST IV

J-13 MATER EXPERIMENT MITH TUFF NRO STAINLESS STEEL

DUPF GLASS (MONOLITH)

RAW DATA TABULATION - LEACHATE AULLYSIS

DAY 28 (PAGE 2)

Analytical Result

SAMPLE ID

13

14

Start Experiant

Stop Intefral

Initial $\mathrm{pH}$

Final pH

Diff (pH)

Spec SA $\left(\mathrm{m}^{2}\right)$

Volume (g)

SAi:

Temp $\left({ }^{\circ} \mathrm{C}\right)$

Initial Weight(g)

Final Weight $(g)$

Weight Loss(g)

\%leach Loss

Initial Spec Ut(g)

Final Spec $\mathrm{Ht}(g)$

Spec Wt Loss (g)

Rock Weight(g)

$\begin{array}{ll}5 / 7 / 84 & 5 / 7 / 84 \\ 6 / 4 / 84 & 6 / 4 / 84 \\ 7.98 & 7.98 \\ 9.65 & 9.58 \\ 1.67 & 1.60 \\ 583 . & 599 \\ 11.67 & 11.98 \\ 0.5 & 0.5 \\ 90.0 & 90.0 \\ 52.10 & 52.34 \\ 51.15 & 51.65 \\ 0.95 & 0.69 \\ 1.82 & 5.76 \\ 1.4772 & 1.2733 \\ 1.4771 & 1.2734 \\ 0.0001 & 0.0001+ \\ 0.58 & 0.60\end{array}$

CONSTITUENT AMALYSIS(mg/l ZER)

Analytical Result

Corrected for Blank

SAMPLE ID

13

14

613

C14

AI
$B$
Fe
LI
Mn
MI
SI
Sr
Zr
$\mathrm{Ca}$
$\mathrm{Mg}$
$\mathrm{Ma}$

.588

.673

.435

.051

.852

.051

.946

.003

$<.008$

56.5

.003

$<.008$

0.015

54.9

0.025

C.002

$<.002$

8.12

8.54

.025

.056

62.2

.069

.154

.100

.112

.024

.803

.001

$<.008$

4.0

$-.009$

$<.002$

1.5

.013

5.1

<.008

2.4

.001

$<.002$

1.1

.044

4.1 
TEST IV

J-13 MATER EXPERIMERT UITH TUFF NWD STAINLESS STEEL

DUPF GLASS (MONOLITH)

RAW DATA TABULATION - LEACHATE AMALYSIS

DAY 57

Analytical Result

SAPLE ID

6

15

Start Experiment

\begin{tabular}{ll}
\multicolumn{1}{c}{6} & \multicolumn{1}{c}{15} \\
$5 / 7 / 84$ & $5 / 7 / 84$ \\
$7 / 3 / 84$ & $7 / 3 / 84$ \\
7.98 & 7.98 \\
9.45 & 9.36 \\
1.47 & 1.38 \\
408. & 597. \\
13.62 & 11.94 \\
0.3 & 0.5 \\
90.0 & 90.0 \\
53.75 & 52.35 \\
52.47 & 51.73 \\
1.28 & 0.62 \\
9.40 & 5.19 \\
0.9309 & 1.4150 \\
0.9315 & 1.4153 \\
$0.0006+$ & $0.0003+$ \\
0.68 & 0.60
\end{tabular}

Stop Interval

Initial pH

Final pH

Diff (pH) ${ }_{\text {Spec }}{ }^{2}$ )

Volume (g)

SNV

Temp $\left({ }^{\circ} \mathrm{C}\right)$

Initial Weight(g)

Final Weight $(g)$

Weight Loss(g)

KLeach Loss

Initial Spec Ut(g)

Final Spec Ht(g)

Spec Wt Loss $(g)$

Rock Weight $(g)$

CONSTITUENT ANALLYSIS(mg/liter)

Analytical Result

SAMPLE ID

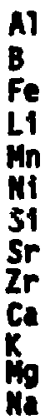

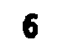

0.059

0.394

0.028

0.547

0.003

0.023

61.4

0.070

0.005

9.39

30.2

0.009

60.0
15

0.553

0.437

0.039

1.08

0.002

<.008

57.0

0.068

<.002

7.88

37.1

0.009

59.6
Corrected for Blank

C6 $\quad$ C15

$\begin{array}{ll}-.462 & 0.032 \\ 0.072 & 0.115 \\ 0.010 & 0.021 \\ 0.491 & 1.024 \\ 0.000 & -.001 \\ 0.023 & -.008 \\ 0.9 & -3.5 \\ 0.009 & 0.007 \\ 0.005 & <.002 \\ 1.84 & 0.33 \\ 12.0 & 18.9 \\ 0.003 & 0.003 \\ 0.2 & -.2\end{array}$


TEST IV

J-13 MATER EXPERIMENT MITH TUFF MND STAIMLESS STEEL

DHPF GLASS (MONOLITH)

RAN DATA TABULATION - LEACHATE MIRLYSIS

DAY 91

Analytical Result

\begin{tabular}{|c|c|c|c|c|c|}
\hline IPLE ID & 7 & 8 & - & 26 & 17 \\
\hline 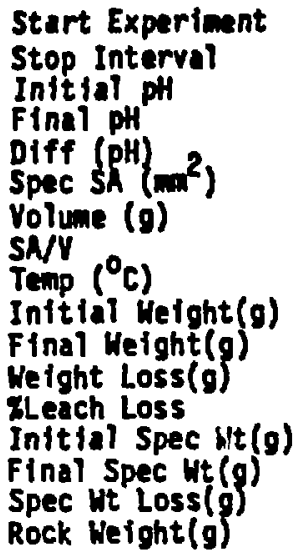 & $\begin{array}{l}5 / 7 / 84 \\
8 / 6 / 84 \\
7.98 \\
9.38 \\
1.40 \\
464 . \\
15.47 \\
0.3 \\
90.0 \\
55.65 \\
54.28 \\
1.37 \\
8.86 \\
1.0007 \\
1.0008 \\
+0.0001 \\
0.77\end{array}$ & $\begin{array}{l}5 / 7 / 84 \\
8 / 6 / 84 \\
7.98 \\
9.51 \\
2.53 \\
452 . \\
15.08 \\
0.3 \\
90.0 \\
54.95 \\
53.28 \\
1.67 \\
11.07 \\
0.8549 \\
0.8549 \\
+0.0004 \\
0.75\end{array}$ & & $\begin{array}{l}5 / 7 / 84 \\
8 / 6 / 84 \\
7.98 \\
9.43 \\
1.45 \\
618 . \\
12.36 \\
0.5 \\
90.0 \\
53.00 \\
52.00 \\
1.00 \\
8.09 \\
1.6354 \\
1.6363 \\
+0.0000 \\
0.62\end{array}$ & $\begin{array}{l}5 / 7 / 84 \\
8 / 6 / 84 \\
7.98 \\
9.47 \\
1.49 \\
615 . \\
12.31 \\
0.5 \\
90.0 \\
54.00 \\
53.00 \\
1.00 \\
8.12 \\
2.7133 \\
2.7133 \\
0.0002 \\
0.62\end{array}$ \\
\hline
\end{tabular}

\section{CONSTITUENT AMALYSIS(mg/I ItER)}

Analytical Result

Corrected for Biank

\begin{tabular}{|c|c|c|c|c|c|c|c|c|}
\hline & 7 & 8 & 16 & 17 & C7 & $\infty$ & $\mathrm{Cl6}$ & C17 \\
\hline $\begin{array}{l}\text { Al } \\
\text { B } \\
\text { Fe } \\
\text { Li } \\
\text { Hi } \\
\text { Si } \\
\text { Sr } \\
\text { Zr } \\
\text { Ca }\end{array}$ & $\begin{array}{l}.477 \\
.395 \\
.053 \\
.778 \\
.008 \\
64.7 \\
0.055 \\
0.003 \\
8.72 \\
26.8 \\
.042 \\
60.8\end{array}$ & $\begin{array}{l}.713 \\
.409 \\
.077 \\
.760 \\
8.008 \\
66.4 \\
0.054 \\
0.002 \\
9.04 \\
22.0 \\
.019 \\
63.1\end{array}$ & $\begin{array}{l}.501 \\
.430 \\
.015 \\
1.18 \\
6.008 \\
62.9 \\
0.047 \\
0.002 \\
8.19 \\
20.7 \\
.013 \\
62.7\end{array}$ & $\begin{array}{l}.475 \\
.445 \\
.032 \\
1.31 \\
6.008 \\
62.5 \\
0.052 \\
0.002 \\
6.91 \\
20.7 \\
.013 \\
62.6\end{array}$ & $\begin{array}{l}-.0267 \\
-.036 \\
.027 \\
.709 \\
<.008 \\
-10.5 \\
-.008 \\
.001 \\
-1.88 \\
5.2 \\
.026 \\
-11.8\end{array}$ & $\begin{array}{l}-.030 \\
-.022 \\
.051 \\
.091 \\
-.008 \\
-8.8 \\
-.009 \\
.000 \\
-1.56 \\
0.4 \\
.003 \\
-9.6\end{array}$ & $\begin{array}{l}-.242 \\
-.001 \\
-.012 \\
1.11 \\
6.008 \\
-12.3 \\
-.016 \\
.000 \\
-2.41 \\
-.9 \\
-.003 \\
-9.9\end{array}$ & $\begin{array}{l}-.332 \\
.014 \\
.006 \\
1.24 \\
-.008 \\
-12.7 \\
-.021 \\
.000 \\
-1.69 \\
-.9 \\
-.003 \\
-10 .\end{array}$ \\
\hline
\end{tabular}

